\title{
Asymmetric and axisymmetric dynamics of tropical cyclones
}

\author{
J. Persing ${ }^{1}$, M. T. Montgomery ${ }^{2}$, J. C. McWilliams ${ }^{3}$, and R. K. Smith ${ }^{4}$ \\ ${ }^{1}$ Department of Meteorology, Naval Postgraduate School, Monterey, CA, USA \\ ${ }^{2}$ Department of Meteorology, Naval Postgraduate School, Monterey, CA, USA \\ ${ }^{3}$ Department of Atmospheric and Oceanic Sciences, University of California at Los Angeles, Los Angeles, CA, USA \\ ${ }^{4}$ Meteorological Institute, Ludwig Maximilians University of Munich, Munich, Germany \\ Correspondence to: M. T. Montgomery (mtmontgo@nps.edu)
}

Received: 26 October 2012 - Published in Atmos. Chem. Phys. Discuss.: 22 May 2013

Revised: 22 August 2013 - Accepted: 9 November 2013 - Published: 18 December 2013

\begin{abstract}
We present the results of idealized numerical experiments to examine the difference between tropical cyclone evolution in three-dimensional (3-D) and axisymmetric (AX) model configurations. We focus on the prototype problem for intensification, which considers the evolution of an initially unsaturated AX vortex in gradient-wind balance on an $f$ plane. Consistent with findings of previous work, the mature intensity in the 3-D model is reduced relative to that in the AX model. In contrast with previous interpretations invoking barotropic instability and related horizontal mixing processes as a mechanism detrimental to the spin-up process, the results indicate that 3-D eddy processes associated with vortical plume structures can assist the intensification process by contributing to a radial contraction of the maximum tangential velocity and to a vertical extension of tangential winds through the depth of the troposphere. These plumes contribute significantly also to the azimuthally averaged heating rate and the corresponding azimuthal-mean overturning circulation.

The comparisons show that the resolved 3-D eddy momentum fluxes above the boundary layer exhibit counter-gradient characteristics during a key spin-up period, and more generally are not solely diffusive. The effects of these eddies are thus not properly represented by the subgrid-scale parameterizations in the AX configuration. The resolved eddy fluxes act to support the contraction and intensification of the maximum tangential winds. The comparisons indicate fundamental differences between convective organization in the 3$\mathrm{D}$ and $\mathrm{AX}$ configurations for meteorologically relevant forecast timescales. While the radial and vertical gradients of the system-scale angular rotation provide a hostile environment for deep convection in the 3-D model, with a corresponding
\end{abstract}

tendency to strain the convective elements in the tangential direction, deep convection in the AX model does not suffer this tendency. Also, since during the 3-D intensification process the convection has not yet organized into annular rings, the azimuthally averaged heating rate and radial gradient thereof is considerably less than that in the AX model. This lack of organization results broadly in a slower intensification rate in the 3-D model and leads ultimately to a weaker mature vortex after 12 days of model integration. While azimuthal mean heating rates in the 3-D model are weaker than those in the AX model, local heating rates in the 3-D model exceed those in the AX model and at times the vortex in the 3-D model intensifies more rapidly than AX. Analyses of the 3-D model output do not support a recent hypothesis concerning the key role of small-scale vertical mixing processes in the upper-tropospheric outflow in controlling the intensification process.

In the 3-D model, surface drag plays a particularly important role in the intensification process for the prototype intensification problem on meteorologically relevant timescales by helping foster the organization of convection in azimuth. There is a radical difference in the behaviour of the 3-D and AX simulations when the surface drag is reduced or increased from realistic values. Borrowing from ideas developed in a recent paper, we give a partial explanation for this difference in behaviour.

Our results provide new qualitative and quantitative insight into the differences between the asymmetric and symmetric dynamics of tropical cyclones and would appear to have important consequences for the formulation of a fluid dynamical theory of tropical cyclone intensification and mature intensity. In particular, the results point to some 
fundamental limitations of strict axisymmetric theory and modelling for representing the azimuthally averaged behaviour of tropical cyclones in three dimensions.

\section{Introduction}

Observations show that tropical cyclones are highly asymmetric during their intensification phase. Only the most intense storms exhibit a strong degree of axial symmetry and, even then, only in their inner-core region. Observations show also that rapidly developing storms are accompanied by "bursts" of convection, presumably driven by significant local buoyancy. In general, deep convection is maintained by moisture fluxes at the ocean-air interface and supported by an unstable thermodynamic environment. The convection consumes local convective available potential energy (CAPE) in the column above the moistened boundary layer and stirs remnant anomalies of equivalent potential temperature $\left(\theta_{\mathrm{e}}\right)$ through the high straining motions that it generates within and above the boundary layer. Both of these processes create an environment less favourable for future convective episodes until the boundary layer re-establishes some ambient level of instability as a result of sea-to-air moisture fluxes (Nguyen et al., 2011).

When buoyant convection occurs in a environment with non-zero vertical vorticity, updraughts will amplify the vorticity by the process of vortex-tube stretching (e.g., Julian et al., 1996; Sprague et al., 2006; Wissmeier and Smith, 2011).

There is accumulating observational and numerical modelling evidence affirming the hypothesis that deep convection in pre-depression disturbances and tropical cyclones acts to spin up localized cyclonic vorticity anomalies in the lower troposphere (Reasor et al., 2005; Bell and Montgomery, 2010; Sippel et al., 2006; Raymond and Lopez, 2011; Sanger et al., 2013, Kilroy and Smith, 2012). The role of these rotating deep convective clouds and their aggregation in the amplification of the larger-scale vortex has been the subject of recent numerical and theoretical investigations (Hendricks et al., 2004; Montgomery et al., 2006; Nguyen et al., 2008; Shin and Smith, 2008; Fang and Zhang, 2010; Braun et al., 2010; Gopalakrishnan et al., 2011; Schecter, 2011) and is reviewed briefly below.

\subsection{Role of vortical deep convection in tropical cyclones}

The studies referred to above suggest that vortical updraughts have typical horizontal length scales of 10 to $20 \mathrm{~km}$ and lifespans of an hour or more. The magnitude of the vorticity amplification above its ambient value is up to $1-2$ orders in the lowest $5 \mathrm{~km}$ of the troposphere. This vorticity outlives the convection that produced it and the like-signed vortical remnants tend to aggregate in a quasi two-dimensional manner with a corresponding upscale vorticity cascade together with a segregation and weakening of the convectively gener- ated anticyclonic vorticity, at least above the boundary layer. Some of the positive remnants are intensified further by subsequent convective episodes.

When the system-scale circulation becomes sufficiently strong, vorticity remnants tend to become axisymmetrized by the associated angular shear flow. In addition, systemscale inflow forced by the aggregate latent heating from the convective elements leads to an inward advection of both system-scale absolute vorticity and convectively enhanced vorticity. Stokes' theorem applied to a fixed area surrounding the convection implies that there will be an accompanying increase in strength of the system-scale circulation on account of the import of net ambient absolute vorticity into it. When applied to a fixed area within the convective region, the import ${ }^{1}$ also of net convectively enhanced cyclonic vorticity into the area will lead to an increase in the circulation within the convective region. As the near-surface circulation increases progressively in strength, there is some increase in the surface moisture fluxes (Montgomery et al., 2009), which will accelerate the replenishment of ambient CAPE. However, Montgomery et al. (2009) showed that it is not necessary that the moisture fluxes continue to increase with surface wind speed, hitherto believed to be an essential element in the intensification process (Rotunno and Emanuel, 1987; Emanuel et al., 1994).

The emerging paradigm of tropical cyclone spin-up as articulated above recognizes the intrinsic fluid dynamics and thermodynamics of rotating deep convection as well as its collective effects in producing system-scale inflow. It recognizes also the potential role of vortex Rossby waves (VRWs ${ }^{2}$ ) and their wave-mean and wave-wave interaction as well as their coupling to the boundary layer and convection (Chen and Yau, 2001; Wang, 2002a, b; Chen et al., 2003; Martinez, 2008; Martinez et al., 2010, 2011). In other words, the tropical cyclone intensification process generally comprises a turbulent system of rotating, deep moist convection and vortex Rossby waves. A recent review of the new paradigm and its relationship to previous ones is given by Montgomery and Smith (2013).

\footnotetext{
${ }^{1}$ The stretching and thereby amplification of ambient (or systemscale) vorticity by convection by itself does not lead to an increase in the circulation so defined, because stretching leads to a contraction in the areal extent of the amplified vorticity (see Haynes and McIntyre, 1987).

${ }^{2}$ VRWs are analogues of planetary Rossby waves (e.g., Holton, 2004), but propagate on the potential vorticity gradient of the system-scale vortex and are affected by the corresponding differential rotation in the radial and vertical direction (Shapiro and Montgomery, 1993; Guinn and Schubert, 1993; Montgomery and Kallenbach, 1997; Schubert et al., 1999; Möller and Montgomery, 2000; McWilliams et al., 2003).
} 


\subsection{An axisymmetric perspective}

There is an extensive literature describing the axisymmetric dynamics of tropical cyclone intensification (Ooyama, 1969; Carrier, 1971; Ooyama, 1982; Anthes, 1982; Shapiro and Willoughby, 1982; Hack and Schubert, 1986; Rotunno and Emanuel, 1987; Emanuel, 1989, 1995, 1997, 2012; Willoughby, 1990; Nguyen et al., 2002; Smith et al., 2011) and maximum possible intensity for a given thermodynamic environment (Miller, 1958; Malkus and Riehl, 1960; Carrier et al., 1971; Emanuel, 1986, 2012; Bister and Emanuel, 1998; Camp and Montgomery, 2001; Emanuel et al., 2004; Bryan and Rotunno, 2009a, b; Emanuel and Rotunno, 2011).

In axisymmetric models, all effects of the flow asymmetries must be parameterized in terms of azimuthally averaged variables. Previous studies have suggested that flow asymmetries generally contribute adversely to the intensification rate and the mature hurricane intensity (e.g., Yang et al., 2007; Bryan et al., 2010). One type of flow asymmetry results from the barotropic breakdown of an unstable ring vortex (e.g., Michalke and Timme, 1967; Rotunno, 1979; Vladimirov and Tarasov, 1979; Schubert et al., 1999). The ensuing potential vorticity redistribution process has been shown to weaken the maximum tangential wind while simultaneously spinning up the flow within the eye towards solid body rotation (Schubert et al., 1999). On the basis of these findings, the net effect of the asymmetries has been assumed to be the down-gradient mixing of momentum and (local) buoyancy. This diffusivelike mixing process has been hypothesized to yield a reduction of the intensification rate and a weakening of the intensity of the storm at maturity relative to its axisymmetric counterpart (Bryan et al., 2010).

In the azimuthal-mean perspective, deep convection contributes in the aggregate to a system-scale radial gradient of positive latent heating that spans the troposphere and is maximized slightly above the mid-troposphere. This heating gradient leads to an azimuthally averaged inflow in the lower half of the troposphere and outflow above. The deep convection contributes collectively to spinning up the tropical cyclone vortex through the radial convergence of azimuthally averaged absolute angular momentum $M=r V+$ $\frac{1}{2} f r^{2}$, where $r$ is the radius, $V$ is the azimuthally averaged tangential wind speed, and $f$ is the Coriolis parameter. Above the frictional boundary layer, $M$ is materially conserved, assuming that eddy processes are unimportant. Since $M$ is proportional to the absolute circulation for a circular loop around the vortex, the material conservation of $M$ implies a concomitant increase of aerially averaged absolute vorticity within a closed circuit moving with the azimuthally averaged inflow. This mechanism has been articulated previously by many authors (e.g., Willoughby, 1979; Shapiro and Willoughby, 1982). It explains why the vortex expands in radial extent as measured by an increase in tangential winds beyond the radius of maximum winds and may be interpreted in terms of axisymmetric balance dynamics (Bui et al., 2009; Smith et al., 2011).

Although $M$ is not materially conserved in the boundary layer, large tangential wind speeds can be achieved there if the radial inflow is sufficiently large to bring the air parcels to small radii with minimal loss of $M$. This spin-up mechanism, while coupled to the interior flow via the radial pressure gradient at the top of the boundary layer, is tied fundamentally to the dynamics of the boundary layer, where the flow is not in gradient wind balance over a substantial radial span (Smith et al., 2009).

\subsection{How different is tropical cyclone dynamics in three- dimensional and axisymmetric models?}

Apart from the assumed downgradient role of all asymmetric motions (including vortical convection and VRWs), the apparent success of AX models has supported a view that, in the absence of vertical shear and vortex translation, the structure and evolution of the azimuthally averaged fields in the 3-D model are captured by a strictly AX model (Anthes et al., 1971; Emanuel, 1991, 1999; Bryan et al., 2010).

An open question remains as to whether there are other important differences between 3-D tropical cyclones and their purely AX counterparts. An indication, by analogy, of a deficiency in a symmetric depiction of convection, itself, is the excessive convective entrainment rate in a two-dimensional planetary boundary layer with vertical shear relative to a 3D model (Moeng et al., 2004). These findings suggest a hypothesis that axisymmetric convection occurring in concentric sheets is overly efficient in generating buoyancy fluxes compared to 3-D convection in isolated plumes, leading in the hurricane context to excessive condensation heating and an overly rapid spin-up; we will see that this hypothesis is broadly supported by our 3-D and AX comparisons.

Two notable studies attempting to address the foregoing question in the tropical cyclone context are those of Nolan and Grasso (2003) and Nolan et al. (2007), who developed a linear theory of heat forcing on an axisymmetric tropical cyclone-like vortex without a secondary circulation. When a balanced vortex is forced by small-amplitude temperature perturbations with 3-D structure motivated by satellite observations and numerical simulations of tropical cyclones, the results indicate that purely asymmetric heat forcing caused weakening of the vortex circulation. They found also that the evolution of the mean vortex with both symmetric and asymmetric components of diabatic forcing is closely approximated by the symmetric response to the azimuthally averaged heating rate. The weakening of the symmetric vortex associated with the asymmetric temperature perturbations occurs on account of a ".. transient growth of the perturbations by downgradient transport of momentum across the radial and vertical shears of the symmetric wind field". While much of this energy is returned to the mean vortex at long times, there appears to be a net loss of mean energy due 
to the axisymmetrization and downscale enstrophy cascade as the potential vorticity anomalies of the perturbations undergo spiral wind-up around the mean vortex. These findings are limited to small-amplitude perturbations and also by the choice to consider only the temperature part of the convective elements. Nevertheless, they motivate an examination of the differences in the mean heating rate of the 3-D and AX models within the context of rotating-convective structures of finite amplitude that possess both temperature and vorticity anomalies.

Another prominent study examining the foregoing question is that of Yang et al. (2007), who used a numerical model configuration that was nearly identical in the 3-D and AX versions. They suggested three effects that contribute to making the mature vortex in the 3-D model roughly $10 \%$ weaker than that in the AX model. The first is that the intensity is controlled principally through a modified entropy imbalance in the sub-cloud layer of the vortex at the radius of maximum tangential wind. The diagnostic model for the entropy imbalance and its connection to the maximum gradient wind is via the energetically based steady-state potential intensity theory of Emanuel (1995) without dissipative heating. The second is that the eyewall in the 3-D model is less tilted than that in the AX model, which they argued leads to less evaporation/modification of the inflowing air and a reduced entropy imbalance at the surface in the 3-D model. The third is similar to the simple view of "down-gradient" momentum mixing described above and argues that the eddies lead to a direct spin-down of tangential winds at the RMW.

We have several concerns about Yang et al.'s explanation. First, Emanuel's potential intensity theory is only a theory for maximum gradient wind (Emanuel, 1986), and recent work has identified a fundamental weakness by not representing the intrinsically unbalanced dynamics of the underlying boundary layer and the resulting implications for the maximum radial and tangential velocity of the vortex (Smith et al., 2008; Bryan and Rotunno, 2009a, b). A second weakness has been pointed out by Emanuel (2012), himself, who noted that the assumption of a constant outflow temperature in the steady-state theory of Emanuel (1986) is poor, requiring a major revision (Emanuel and Rotunno, 2011). Thus the first leg of the Yang et al., model appears to be fundamentally challenged. Second, in our view, the authors do not provide a satisfactory explanation as to why an eyewall in the 3-D model should be less-tilted than in the equivalent AX model. Finally, the authors gave no apparent attention to the differences in the convective organization (convection and vorticity aggregation, upscale cascade of vorticity, etc.) in the two models.

\subsection{The present study}

In this paper we address the question posed in the title of Sect. 1.3. In particular, we compare selected azimuthally averaged fields of the 3-D model with the corresponding fields in the AX model, both during intensification and in the mature stage for the prototype intensification problem studied by Nguyen et al. (2008). This problem considers the evolution of a prescribed, initially cloud-free, axisymmetric, baroclinic vortex in a quiescent environment over a warm ocean on an $f$ plane. Specific fields to be examined include all three velocity components, the heating rate, terms in the tangential momentum equation, a forcing function controlling the balanced spin-up of the bulk vortex, subgrid-scale and resolved Reynolds stresses, and horizontal and vertical eddy diffusivities. Another field examined is a gradient Richardson number, which has been hypothesized recently to be a critical component of tropical cyclone intensification. In addition to presenting these comparisons, we explore and discuss the implications of the results.

The remainder of the paper is structured as follows. Section 2 summarizes the model setup and defines the initial vortex structure, together with the temperature and moisture profiles used for the numerical experiments. Section 3 gives an overview of the simulation results. Section 4 presents an analysis of the balanced spin-up of the bulk vortex (outside of the boundary layer and outflow layer). Section 5 examines the tangential momentum equation for both 3-D and AX systems. Section 6 takes a closer look at the subgrid scale and Reynolds stresses in the two model configurations. Some consequences of the differences in 3-D and AX systems found above are illustrated in Sect. 7 with a specific series of numerical experiments in which the surface drag coefficient is varied. Section 8 presents our conclusions.

\section{The numerical model}

The experiments are performed using the numerical model CM1 version 14 , a non-hydrostatic and fully compressible cloud model (Bryan and Fritsch, 2002) ${ }^{3}$. Over the sea, as is the case here, the terrain-following coordinate system simplifies to regular Cartesian coordinates. An attractive feature of this model is that it has options for being executed in AX or 3-D configurations.

In the 3-D configuration there are prediction equations for the three components of the velocity vector $\boldsymbol{u}$, water vapour $q_{\mathrm{v}}$, suspended liquid $q_{\mathrm{l}}$, perturbation Exner function $\pi^{\prime}=\left(p^{\prime} / p_{0}\right)^{R / c_{\mathrm{p}}}$, and perturbation potential temperature $\theta^{\prime}$, where perturbation quantities are defined relative to a prescribed hydrostatic basic state. Here $c_{\mathrm{p}}$ is the specific heat of dry air at constant pressure $p, R$ is the gas law constant for dry air, and $p_{0}=10^{5} \mathrm{~Pa}$ is a reference pressure. For simplicity, ice microphysical processes are neglected. The

\footnotetext{
${ }^{3}$ For a complete description of the three-dimensional model and variable definitions see the technical document "The governing equations for CM1", available for download at http://www.mmm. ucar.edu/people/bryan/cm1 and also available from G. Bryan. For a complete description of the axisymmetric version of CM1, see the paper by Bryan and Rotunno (2009a).
} 
reference sounding is a nearly moist-neutral sounding generated from the axisymmetric Rotunno-Emanuel (1987) model. Near-neutral (very low CAPE) soundings have served as a prototype in idealized studies since Emanuel (1986) demonstrated that ambient CAPE is unnecessary for tropical cyclone maintenance and since many numerical simulations (e.g. Rotunno and Emanuel, 1987; Montgomery et al., 2009) are able to simulate intensification with very little environmental CAPE. Persing and Montgomery (2005) presented a suite of axisymmetric simulations where approximately the same intensity was found for a wide range of environmental soundings with varying CAPE.

On the numerical side the advection terms are calculated in flux form. The pressure gradient force per unit mass takes the form $\boldsymbol{F}_{\mathrm{P}}=-c_{\mathrm{p}} \theta_{\rho} \nabla \pi$, where the pertinent density potential temperature $\theta_{\rho}$ is defined as $\theta_{\rho}=\theta\left(1+q_{\mathrm{v}} / \epsilon\right) /\left(1+q_{\mathrm{v}}+q_{1}\right)$, with $\epsilon=R / R_{\mathrm{V}}$, where $R_{\mathrm{V}}$ is the gas constant for water vapor. The vertical momentum equation includes a buoyancy force per unit mass $B=g\left(\theta_{\rho}-\theta_{\rho 0}\right) /\left(\theta_{\rho 0}\right)$, where $\theta_{\rho 0}(z)$ is the basic state profile of $\theta_{\rho}$, and $g$ is the gravitational acceleration. The tendencies of $\theta^{\prime}$ and $\pi^{\prime}$ are calculated using the mass- and energy-conserving equations derived by Bryan and Fritsch (2002). For simplicity, dissipative heating is not included (cf. Bister and Emanuel, 1998) and should not alter any of the conclusions or interpretations herein. The calculations are carried out on an $f$ plane with the Coriolis parameter $f=5 \times 10^{-5} \mathrm{~s}^{-1}$, corresponding to $20^{\circ} \mathrm{N}$.

Radiative effects are represented by adopting a simple Newtonian cooling approximation capped at $2 \mathrm{~K}$ per day, following Rotunno and Emanuel (1987). This approximation serves as a simple expedient to parameterize the radiativeconvective equilibrium process, which operates to maintain the ambient tropical sounding over realistic forecast timescales of several days. In choosing this particular setup, we purposely omit (1) the more complex cloud-radiative feedback processes in the infrared wavelengths that have been suggested to accelerate the intensification process (e.g., Hakim, 2011; Nicholls and Montgomery, 2012) and (2) the negative ocean feedback associated with storm-induced upwelling of colder ocean water below the storm that tends to retard the intensification process (e.g., Emanuel et al., 2004). To some extent, these two effects act in opposition and it is reasonable to omit both in our first step in developing a basic fluid dynamical understanding of the differences between 3-D and AX tropical cyclones.

The outer lateral boundaries are open with a radiative boundary condition (Durran and Klemp, 1983). In order to mitigate the reflection of internal gravity waves from the upper boundary, a Rayleigh damping layer is added at heights above $20 \mathrm{~km}$. The surface temperature is $299.3 \mathrm{~K}$ (based on the value used in the study by Rotunno and Emanuel, 1987). A simple rainfall scheme is used in which rain has a fixed fall speed of $7 \mathrm{~m} \mathrm{~s}^{-1}$.

Subgrid-scale turbulence is represented by choosing the available option ("iturb=3") in the model, which is designed for problems that do not resolve any part of the turbulent Kolmogorov inertial range. This option requires the external specification of horizontal and vertical mixing lengths $l_{\mathrm{h}}$ and $l_{\mathrm{v}}$, which for simplicity are assumed constant in both space and time. The scheme follows Smagorinsky (1963) and Lilly (1962), except that different eddy viscosities must be used for the horizontal and vertical directions. The flowdependent momentum diffusivities in the horizontal and vertical directions are specified as follows: $K_{\mathrm{m}, \mathrm{h}}=l_{\mathrm{h}}^{2} S_{\mathrm{h}}$ and $K_{\mathrm{m}, \mathrm{v}}=l_{\mathrm{v}}^{2} S_{\mathrm{v}} \sqrt{1-R i / P r}$, where the " $\mathrm{m}$ " subscript refers to momentum, and the second subscript "h" or "v" refer to the horizontal and vertical directions, $S_{\mathrm{h}}$ and $S_{\mathrm{v}}$ denote the terms found in the total deformation, $S$, that involve the horizontal and vertical flow components, $R i=N_{\mathrm{m}}^{2} / S^{2}$ is the moist Richardson number, $N_{\mathrm{m}}^{2}$ is the moist Brunt-Väisälä frequency, and $P r$ is the Prandtl number (set to unity in this option). In this scheme, the vertical eddy diffusivity is proportionally reduced in regions with positive moist Richardson number and the heat and momentum diffusivities are taken to be identical, $K_{\mathrm{h}}=K_{\mathrm{m}}$. Whenever $R i$ exceeds unity, the vertical momentum and vertical heat diffusivities are set to zero.

In our experiments, we adopt a configuration of the 3-D and AX models that share the same corresponding values of horizontal mixing lengths and of vertical mixing lengths. The respective mixing lengths are assumed to apply equally to the parameterized mixing of heat and momentum (e.g., as in Bryan et al., 2010; Bryan, 2012). The choice of the same horizontal mixing lengths in the two models is contrary to that advocated recently by Bryan (2012). He suggests that in order to match the observed intensity of category four and five tropical cyclones one should use an enhanced horizontal eddy-diffusivity in the AX model to represent the total effects of diffusion and eddy processes. For the following two reasons we have some concerns with this argument. First, the underlying physical basis for the "blunt tuning" of the horizontal diffusivity in the AX model is unclear to us. Second, we see the need to use the information about the resolved eddy structures from the 3-D model (i.e., radial and vertical structure and magnitude of resolved Reynolds stresses, and their possible relation to the gradients of the mean fields, etc.) to determine if such a tuning procedure is justified. In this study, we choose to compare the resolved fluid dynamics of the AX and 3-D models using the same formulae for the subgrid-scale processes to ascertain how these systems differ. We see this comparison as a necessary first step to considering how one should revise the AX model to better mimic the dynamics of the 3-D model.

The mixing lengths required in the formulation of the subgrid-scale processes are set to be consistent with recent observations of intense hurricanes at the base of the eyewall region for horizontal wind speeds $\approx 50 \mathrm{~m} \mathrm{~s}^{-1}$ (Zhang and Montgomery, 2012; Zhang et al., 2011): $l_{\mathrm{h}}=700 \mathrm{~m}$ and $l_{\mathrm{v}}=50 \mathrm{~m}$. For reasons given in the foregoing paragraph, the 
horizontal mixing length in the AX model is taken to be the same as in the 3-D model ${ }^{4}$. For the horizontal grid spacing used herein, at least some of the horizontal mixing noted in observations must be resolvable on the 3-D grid mesh.

The parameters determining the exchange of heat and momentum at the air-sea interface are set as follows. The surface exchange coefficients of heat and momentum are taken to be constant in both space and time. The moist enthalpy transfer coefficient $C_{k}$ is set equal to $1.29 \times 10^{-3}$. This value is close to the mean value $\left(1.2 \times 10^{-3}\right)$ derived from the Coupled Boundary Layers/Air-Sea Transfer (CBLAST) experiment (Fig. 6 of Black et al., 2007); Fig. 4 of Zhang et al., 2007), a recent laboratory study (Fig. 1 of Haus et al., 2010) near and slightly above marginal hurricane wind speeds, and an energy and momentum budget analysis of the lowertropospheric eyewall region at major hurricane wind speeds (Bell et al., 2012). The drag coefficient is set to be twice the enthalpy exchange coefficient $C_{\mathrm{D}}=2 \times C_{k}=2.58 \times 10^{-3}$, and is close to the estimated mean value of $C_{\mathrm{D}}=2.4 \times$ $10^{-3}$ from CBLAST-derived observations for major hurricane wind speeds by Bell et al. (2012).

The same initial vortex is used for all simulations. The initial radial and vertical velocity is set to zero. The initial tangential velocity is taken to be in gradient wind balance with a maximum of $13 \mathrm{~m} \mathrm{~s}^{-1}$ at the surface and occurs at a $100 \mathrm{~km}$ radius from the centre of circulation. The tangential velocity varies smoothly in space and tends to zero at large radii: it is effectively zero beyond $400 \mathrm{~km}$ radius and above $z=20 \mathrm{~km}$. The 3-D experiments are conducted with a $3 \mathrm{~km}$ horizontal grid spacing on an interior domain. Further details of the domain sizes and grid stretching are given in Appendix A.

There are two principal numerical simulations: (1) a threedimensional simulation at $3 \mathrm{~km}$ horizontal grid spacing on the interior grid mesh (hereafter called "3D3k") and (2) a corresponding axisymmetric simulation at $3 \mathrm{~km}$ radial grid spacing (hereafter called "AX3k"). The $3 \mathrm{~km}$ grid spacing is sufficient to produce a simulated hurricane with a variety of asymmetries, including eyewall asymmetries necessary to examine the role of asymmetries as opposed to axisymmetric processes in the intensification of a simulated tropical cyclone. Other sensitivity experiments are detailed in Sects. 3 and 7.

\footnotetext{
${ }^{4}$ Although the chosen value of $l_{\mathrm{V}}=50 \mathrm{~m}$ is somewhat below the average mixing length reported by Zhang et al. (2011), it is well within the range of scatter in the observational data. The value of $l_{\mathrm{V}}=50 \mathrm{~m}$ was chosen also by Bryan (2012) to give more "realistic"looking hurricane winds and, in particular, more realistic inflow angles in the boundary layer of his simulated hurricanes in comparison to the observations of Powell et al. (2009). This value of the vertical mixing length (and corresponding vertical eddy momentum diffusivity of approximately $50 \mathrm{~m}^{2} \mathrm{~s}^{-1}$ in the boundary layer region, see Sect. 6 for more) is consistent with recent results reported by Zhang and Drennan (2012) using flux-profile data in the rainband regions of Hurricanes Fabian (2003), Isabel (2003), Frances (2004) and Jeanne (2004).
}

Before presenting specific solutions using a particular model configuration, it should be recalled from the Introduction that tropical cyclone intensification is a turbulent process in which deep convection is a prominent stochastic feature. As a result, one should think of a particular simulation as being just one realization of an ensemble of simulations in which the convection is perturbed. One way that the convection can be perturbed is by adding a stochastic component to the initial moisture in the boundary layer, the amplitude of which is comparable with the errors in observing moisture with current observational platforms (Nguyen et al., 2008; Shin and Smith, 2008). A simpler approach is used here to create a second simulation that is identical to the AX3k simulation, except for the inclusion of a $20 \mu \mathrm{g} \mathrm{kg}^{-1}$ increase in the initial basic state moisture profile over the lowest levels of the simulation. This simulation is denoted as "AX3k" .

Another way that the stochastic nature of convection may materialize is by running the model on a different computational platform. In this case the stochastic nature of truncation error leads to a random component of the convection in comparison with that from the original platform. This sensitivity became apparent when our 3-D calculations were performed on a newer computer, but using the same Fortran code. We have taken the newer 3-D experiments to be our principal experiments, but for comparison purposes we have retained one of our older 3-D experiments and denoted this experiment subsequently as " $3 \mathrm{D} 3 \mathrm{k}^{*}$ " .

\section{Overview of simulated development in the $\mathrm{AX}$ and 3-D models}

\subsection{Vortex evolution}

Figure 1 shows a time series of the maximum tangential velocity $\left(V_{\max }\right)$ and corresponding radius of maximum tangential wind (RMW) simulated in the AX and 3-D model simulations AX3k, AX3k*, 3D 3k and 3D 3k* out to $288 \mathrm{~h}(12$ d). In the 3-D configuration, the maximum velocity plotted is that of the azimuthally averaged tangential velocity anywhere in the domain and the RMW corresponds to the radius of this wind maximum. During both spin-up and maturity the velocity maximum in both 3-D and AX configurations occurs in the height range between $500 \mathrm{~m}$ to $750 \mathrm{~m}$, which is within, but near the top of, the frictional boundary layer of the vortex as defined by the layer of strong radial inflow (Smith and Montgomery, 2010; Zhang et al., 2011). Comparing the two AX runs (with identical initializations save for a very small moisture perturbation as described above) the differences are relatively small (only occasionally more

\footnotetext{
${ }^{5}$ Computational expense and the limited availability of the alternate computation platform dictated the differing treatments, 3-D versus AX. The point of both is that minuscule initial differences result in distinct realizations within an envelope of permissible solutions.
} 

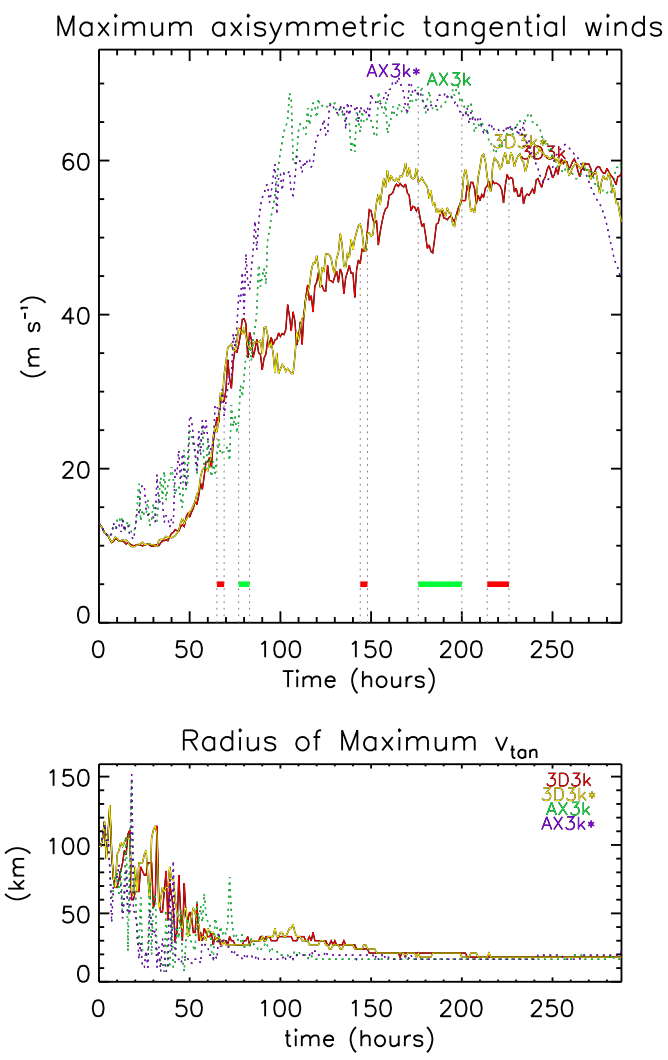

Fig. 1. Maximum azimuthally averaged tangential velocity $\left(V_{\max }\right.$; top) and corresponding radius of maximum tangential wind (RMW; bottom) as a function of time for the four numerical experiments using the CM1 model as described in Sect. 2: the 3D3k simulation (solid red), the $3 \mathrm{D} 3 \mathrm{k}^{*}$ simulation (solid yellow), the AX3k simulation (green dotted), and the AX3k* simulation (purple dotted). In the top panel, the red and green bars bounded by gray, dashed lines denote intervals used in quantitative diagnostic analyses of mean and eddy dynamics presented in Sects. 4 and 6 for the 3D3k and AX3k simulations, respectively: $67 \mathrm{~h}$, centre of first rapid intensification period in the 3D3k simulation; $81 \mathrm{~h}$, centre of rapid intensification period in the AX3k simulation; $153 \mathrm{~h}$, centre of second rapid intensification episode in the 3D3k simulation; $188 \mathrm{~h}$, centre of mature period in the AX3k simulation; and $221 \mathrm{~h}$, centre of mature period in the $3 \mathrm{D} 3 \mathrm{k}$ simulation.

than $5 \mathrm{~m} \mathrm{~s}^{-1}$ ) after accounting for a small time offset during rapid intensification. A quasi-steady maximum intensity is maintained between $130 \mathrm{~h}$ and $190 \mathrm{~h}$ (for approximately $60 \mathrm{~h}$ ) until a slow weakening is evident in both AX runs. Comparing the two 3-D runs (with identical initializations, but carried out on different computer platforms), the differences in $V_{\max }$ are comparatively small during the first $90 \mathrm{~h}$ of integration, which encompasses the first intensification period. After $90 \mathrm{~h}$, there are differences in $V_{\max }$ of up to about $10 \mathrm{~m} \mathrm{~s}^{-1}$, and these differences are typically coherent over longer time periods than in the $\mathrm{AX}$ configuration.
We focus attention now on the differences between the AX3k and the 3D3k simulations ${ }^{6}$. For subsequent analyses and interpretation, we will define the intensification period as a time interval spanning the most rapid rate of increase of the maximum tangential velocity. In experiment AX3k, we take this time interval to be the period between $75 \mathrm{~h}$ and 85 $\mathrm{h}$, and in experiment 3D $3 \mathrm{k}$, the period between $60 \mathrm{~h}$ and 70 $\mathrm{h}$. Since the intensification process in the 3-D experiments spans a comparatively longer time interval, we will consider also a second rapid intensification period in the $3 \mathrm{D} 3 \mathrm{k}$ run between 151 and $155 \mathrm{~h}$.

There is a degree of arbitrariness for defining periods for mature intensity. Here we take periods of maximum intensity that encompass quasi-steady intervals.

A peak intensity of $69.7 \mathrm{~m} \mathrm{~s}^{-1}$ is found in the AX3k model at $198 \mathrm{~h}$ and of $60.0 \mathrm{~m} \mathrm{~s}^{-1}$ in the 3D3k model. Inspection of Fig. 1 shows that the substantial portion of intensification is complete in the AX3k model around $105 \mathrm{~h}$ and in the 3D3k model around $170 \mathrm{~h}$; after which time a fluctuating quasisteady mature stage is observed. Between $200 \mathrm{~h}$ and $250 \mathrm{~h}$, the intensity in the AX model declines slowly to approximately the maximum intensity found in the 3-D model and then continues a gradual decline. In the 3-D model, the intensity declines after $250 \mathrm{~h}$. Longer versions of these simulations (not shown) suggest that the weakening trend continues for many days after the end of these simulations. We have not studied the near coincidence in the intensity in the 3-D and AX simulations starting near $250 \mathrm{~h}$ or the continued weakening trend.

The maximum intensity of the 3-D simulations for the $12 \mathrm{~d}$ simulation is approximately $15 \%$ weaker than that of the AX configurations. In addition, the AX simulations exhibit their peak intensification rate for a longer period of time than the 3-D simulations, although these rates are roughly the same in the two models. Both of these aspects are broadly consistent with prior findings comparing three-dimensional and axisymmetric tropical cyclone simulations (e.g., Yang et al., 2007; Montgomery et al., 2009; Bryan et al., 2010). At early times in both AX and 3-D configurations, the RMW (Fig. 1b) exhibits modest (in 3D3k) and large (in AX3k) fluctuations with time before a more systematic contraction ensues. In the case of the AX simulations, the RMW fluctuates wildly in the early spin-up period, sometimes by more than $100 \mathrm{~km}$. The rapid increase of $V_{\max }$ with time in the AX3k simulation is coincident with a period in which the RMW contracts inwards from 40 to $20 \mathrm{~km}$. Unlike the AX3k simulation, the

\footnotetext{
${ }^{6}$ Strictly speaking, comparing just two simulations can be problematic because of the intrinsic variability associated with deep convection as discussed in the foregoing subsection. It is for this reason that small differences between the two classes of simulations are not emphasized. The detailed dynamics and thermodynamics governing the fluctuations in tangential wind speed is certainly an interesting topic (cf. Nguyen et al., 2011), but for the present purpose we will focus only on the broad aspects determining the basic differences in intensification and intensity between $\mathrm{AX}$ and 3-D simulations.
} 
first rapid intensification period in the 3D3k simulation occurs towards the end of the contraction of the RMW corresponding to the mean tangential wind. The final contraction phase of the RMW in the 3D3k simulation is coincident with the second rapid intensification period identified above. By $250 \mathrm{~h}$, the RMWs for each simulation approach the same radius of approximately $20 \mathrm{~km}$.

It may be worth pointing out that there is a short period of time (near $66 \mathrm{~h}$ ) during the early spin-up period when the rate of spin-up is largest in the 3-D model. We will show later that at about this time the local heating rate exceeds that found in the AX model and there is degree of ring-like organization in the deep convection.

\subsection{Evolution of relative vorticity}

In the foregoing 3-D simulations, the evolution of relative vorticity and vertical velocity is similar to that described in previous work (Hendricks et al., 2004; Montgomery et al., 2006; Nguyen et al., 2008, 2011; Shin and Smith, 2008; Montgomery et al., 2009; Gopalakrishnan et al., 2011). Specifically, there is a brief gestation period in which the boundary layer is moistened by sea-to-air moisture fluxes and during which the vortex intensity weakens by frictional spindown until some air parcels are lifted to their level of free convection. The ensuing deep convection leads to the vertical stretching of local vortex tubes. The presence of a cyclonically rotating background vortex would suggest a tendency for cyclonic vorticity to amplify more rapidly than any anticyclonic vorticity. The cyclonic vorticity so generated tends to aggregate by both vortex mergers and system-scale convergence driven by the collective buoyancy of deep convective clouds ${ }^{7}$. Figures 2 and 3 summarize the evolution in relative vorticity structure at a height of $1 \mathrm{~km}$ in the 3-D simulation and compares it with that in the AX simulation during the intensification phase, a level near the top of the strong inflow layer associated with surface friction. The depictions of Fig. 2 span time periods that contain intervals of peak intensification in each simulation.

The tangential velocities for the 3D3k simulation (left column) and AX3k simulation (right column) are roughly comparable (within $10 \mathrm{~m} \mathrm{~s}^{-1}$ of each other) at the corresponding stages of evolution.

During the first intensification period, the vorticity in the 3-D simulation is an amorphous mass of cyclonic values in the interior, punctuated by several regions of localized in-

\footnotetext{
${ }^{7}$ The present overview neglects the contribution to the material rate of change of vertical vorticity due to vortex-tube tilting, solenoidal generation and subgrid scale diffusion. As discussed elsewhere (e.g., Montgomery et al., 2006 and refs.), one of the more significant of these is the vortex-tube tilting term, which generally contributes small-scale vortex dipole structures on the horizontal scale of the updraughts/downdraughts. The tilting effect must be accounted for in a complete explanation of the material change of vorticity and can alter the simple picture sketched here.
}

tense cyclonic vorticity (Fig. 2a). On the edge of this mass are curved banded features which tend at later times to spiral cyclonically inwards. This cyclonic vorticity region is immersed in a sea of weak cyclonic/anticyclonic vorticity. During the second intensification period (middle column), a contraction of a ring of cyclonic vorticity associated with the eyewall is evident, with asymmetries still present in the vorticity ring corresponding with localized enhancements in the eyewall and in the vorticity bands extending out from the eyewall. In the AX simulation (Fig. 2b), the evolution of the relative vertical vorticity is strikingly different with the vorticity pattern consisting of annular bands of alternating positive and negative values. As in the 3-D simulation, the magnitude of maximum positive relative vorticity in the central disk greatly exceeds the magnitude of the negative vorticity in neighboring rings. The pattern of vorticity rings reflects that of the vertical velocity associated with deep convection in the AX model.

Figure 3 shows a radius-time plot of the relative vorticity at a height of $1 \mathrm{~km}$ for both the 3D3k and AX3k simulations, the vorticity being azimuthally averaged in the 3-D case. In broad terms, both plots exhibit a consolidation of cyclonic vorticity with time, with an elevated maximum near the RMW, whose values are greater there than near the centre of the vortex. The consolidated vortices exhibit a ringlike radial distribution of vertical vorticity as opposed to a monopolar distribution with radius.

The azimuthal-mean vorticity in the 3-D simulation exhibits considerably less spatio-temporal variability than the corresponding AX simulation. This difference can be explained in part by the fact that the convective elements and the vorticity that they generate are averaged azimuthally in the 3-D simulation, whereas the convection and vorticity in the AX model is unfiltered. Another difference between the two simulations is that radial and vertical gradients of the system-scale angular rotation provide a hostile environment for deep convection in the 3-D model, with a corresponding tendency to strain the convective elements in the tangential direction. This process tends to damp the small-scale fluctuations over the larger-scale motions (Melander et al., 1987; Carr and Williams, 1989; Sutyrin, 1990;, Smith and Montgomery, 1995; Rozoff et al., 2006). In contrast, convection in the AX model is not damped by such a process and consequently retains a high degree of noisiness with time. Indeed, there is considerable noise in the vorticity field during the intensification process, which appears to be a consequence of the unrealistic "ring-like" nature of the convection that produces the vorticity.

\subsection{Comparison of surface enthalpy fluxes}

A widely accepted paradigm for tropical cyclone intensification and mature intensity focuses on the role of the ocean energy source via sea-to-air enthalpy fluxes (Emanuel, 2003). Indeed, in their comparison between axisymmetric 

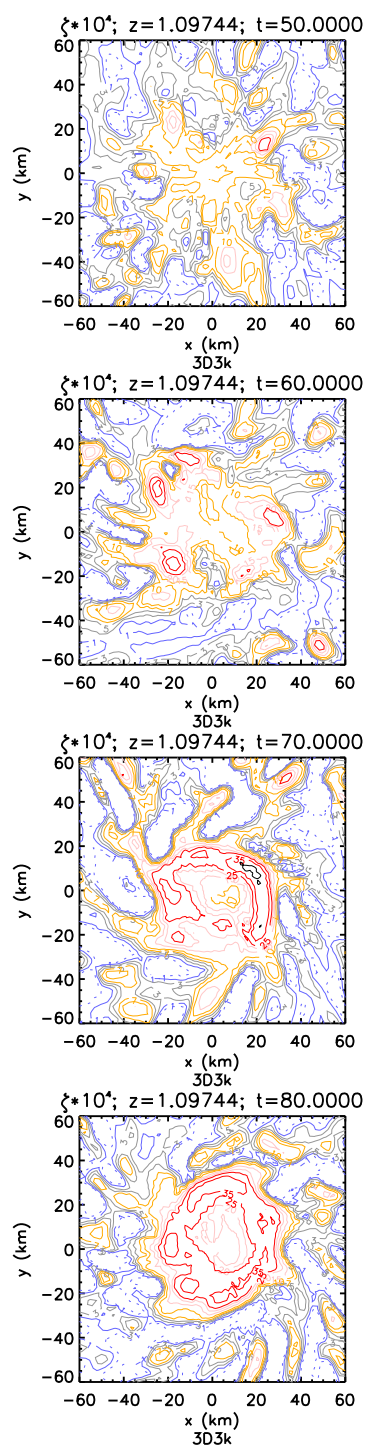
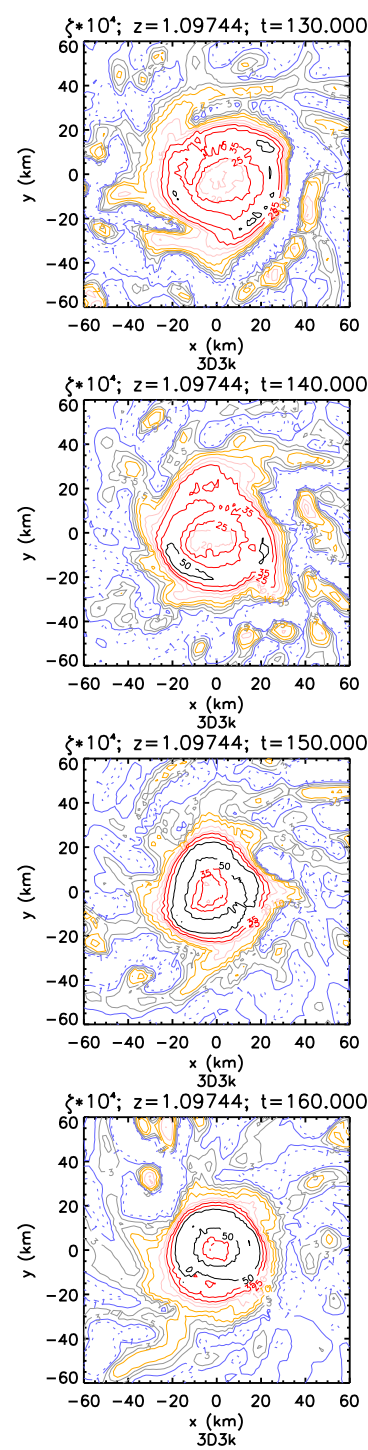
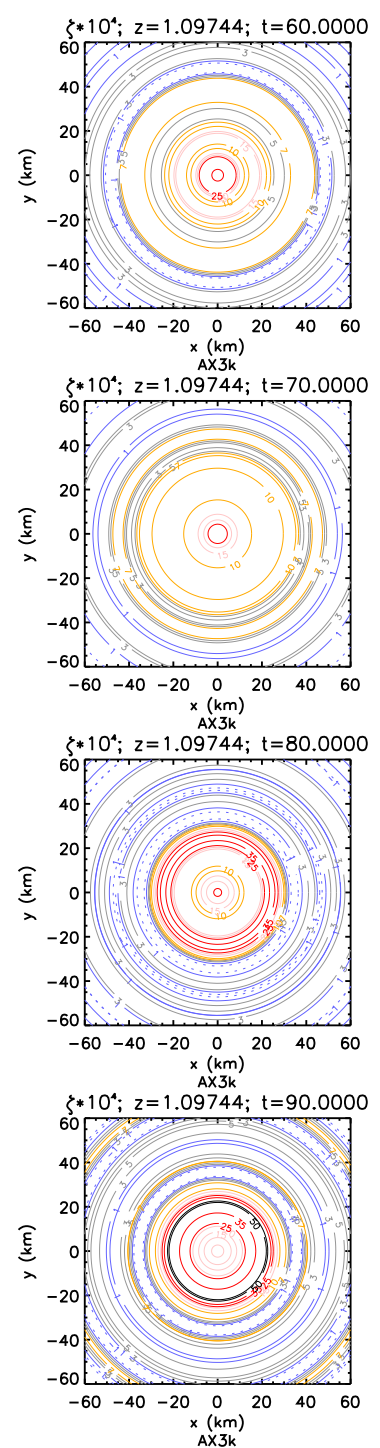

Fig. 2. Contour plots of relative vorticity $\zeta$ times $10^{4}$ at $z=1 \mathrm{~km}$ at a sequence of times (a) and (b) (left-middle) from the simulation 3D3k and (c) (right) from simulation AX3k spanning periods of rapid intensification. The time period shown is between (a) 50-80 $\mathrm{h}$ and (b) $130-160 \mathrm{~h}$ for the 3D3k experiment and (c) 60-90 h in the AX3k experiment. Contours ( $\mathrm{s}^{-1}$ ) are -1 and 1 (blue); 3 and 5 (gray); 7 and 10 (orange); 15 and 20 (pink); 25 and 35 (red); and 50 and 75 (black).

and three-dimensional simulations, Yang et al. (2007) attributed the differences in the mature intensity to the differences in the air-sea disequilibrium and the corresponding total heat flux. For this reason we show in Fig. 4 the radial profile of the azimuthally averaged moist enthalpy flux during the rapid intensification stage and during the mature stage. At each time the radial profile of surface enthalpy flux is maximized near the RMW and increases roughly with the tangential wind speed. The selected times shown for rapid intensification have a similar intensity in both the $3 \mathrm{D} 3 \mathrm{k}$ and AX3k experiments, but at peak intensity the azimuthally averaged enthalpy flux for the AX3k experiment is a little stronger. However, unlike Yang et al. (2007), we do not attribute much physical significance to these differences because the commonly assumed connection between surface fluxes and intensification is tenuous and involves a number of assumptions that are difficult to substantiate (Montgomery et al., 2009). In view of these issues, we believe that a perspective based on both dynamics and thermodynamics is called for. This need provides the motivation for the next section.

\subsection{Comparison of convective heating rates}

One measure for comparing the 3-D and AX simulations is the heating rate associated with deep convection, which is roughly proportional to the vertical velocity (e.g., Holton, 

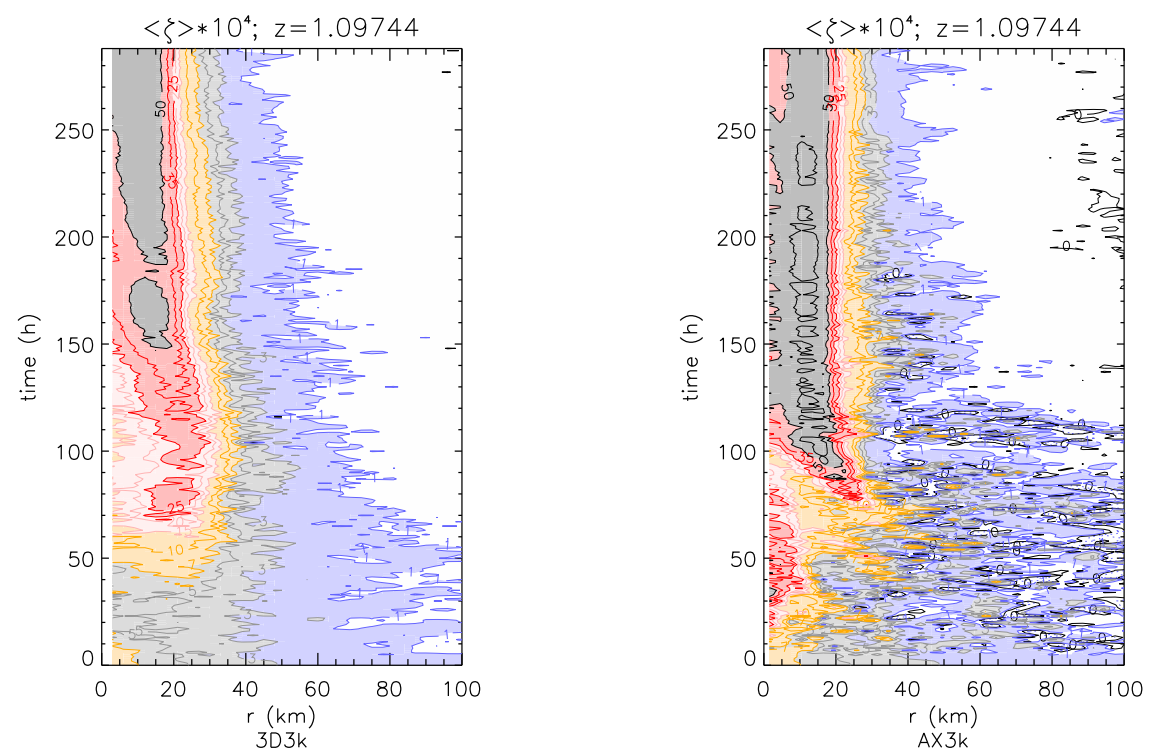

Fig. 3. Radius-time plots of relative vorticity $\zeta$ times $10^{4} \mathrm{~s}^{-1}$ at $z=1 \mathrm{~km}$ (a) (left) from the simulation 3D3k after azimuthal averaging and (b) (right) from simulation AX3k spanning the complete 12 day simulation. Contours $\left(\mathrm{s}^{-1}\right.$ ) are -1 and 1 (blue); 3 and 5 (gray); 7 and 10 (orange); 15 and 20 (pink); 25 and 35 (red); and 50 and 75 (black).

2004). For reasons given later in Sect. 5, the vertical structure and magnitude of the heating rate, as well as its horizontal distribution in relation to the vorticity field of the mean vortex, are pertinent quantities in forcing the spin-up of the bulk vortex. Figure 5 shows radius-height contour plots of the heating rate in the AX3k simulation and the corresponding azimuthally averaged heating rate in the $3 \mathrm{D} 3 \mathrm{k}$ simulation. Shown also is a horizontal cross section of the heating rate in the 3D3k simulation at the height of maximum heating rate $(z \approx 7 \mathrm{~km})$. As in the foregoing subsection, these heating rates are shown at times of peak intensification and peak intensity, respectively, in each simulation. For simplicity, we restrict the quantitative comparison of the convective heating rates between the two configurations to the first intensification interval in the 3D3k experiment. (Similar results are found during the second intensification interval.) Again, the intensities as measured by the maximum azimuthally averaged tangential velocity are not identical at these comparison times, although they are roughly comparable (within $10 \mathrm{~m} \mathrm{~s}^{-1}$ ) with one another in the 3-D and AX simulations.

It is evident from Fig. 5 that the AX simulation has a larger maximum heating rate than the maximum azimuthally averaged heating rate in the 3-D simulation. Peak heating rates occur in the eyewall, typically at a height of around $7 \mathrm{~km}$. The peak heating rate in the AX simulation is two to three times as large as the azimuthally averaged heating rate in the corresponding 3-D simulation (see Fig. 5a and b) both during intensification and the mature stage ${ }^{8}$.

\footnotetext{
${ }^{8} \mathrm{~A}$ factor of two difference between the maximum heating rate in the AX and 3-D models was noted also for the mature stage by
}

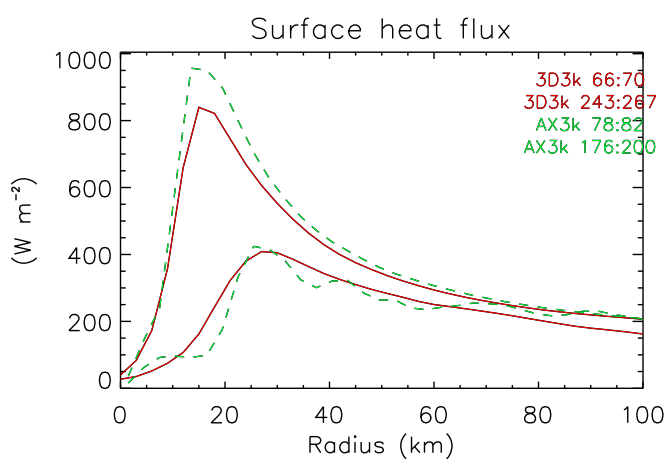

Fig. 4. Radial profile of surface enthalpy flux for the AX3k and 3D $3 \mathrm{k}$ experiments at a time of maximum intensification rate (solid) and when the simulated hurricane has reached mature intensity (dashed).

During intensification in the 3-D simulation, the pattern of convective heating comprises several strong cells loosely organized in bands outside the radius of maximum azimuthally

Yang et al. (2007), their Fig. 4a and 4b and p1172, “... The maximum heating rate in the eyewall in SYM (the AX model) is almost twice as twice large as that in CTL (the 3-D model) ..." However, the interpretation offered therein focused on the effects of downdrafts and the enhanced air-sea disequilibrium in the AX simulation that was suggested to render the AX simulation more intense than the 3-D model. For reasons already noted, the commonly assumed connection between surface fluxes and system-scale intensification is tenuous and involves a number of assumptions that are difficult to substantiate. 
averaged heating rate, and a ring-like organization in the deep convection indicating a developing eyewall updraught (Fig. 5c). Isolated centres in the 3-D simulation exceed the heating rate found in the AX simulation. The fact that only the peak heating rates are comparable to those in the AX simulation explains why the azimuthally averaged heating rates are markedly less than those in the AX simulation. In the mature stage, the heating field in the 3-D simulation is dominated by both an approximately circular inner edge and banded structures, but isolated strong centres of heating are still present (Fig. 5f).

To assess the generality of the above results at different times during the vortex evolution, several times series involving the heating rate are plotted in Fig. 6. The green curve is the maximum heating rate obtained from the AX3k experiment, output every hour. The red curve is the azimuthally averaged maximum heating rate from the $3 \mathrm{D} 3 \mathrm{k}$ simulation, output every hour. The purple curve is the corresponding local maximum heating rate in the 3D3k simulation. The heating rate in the $\mathrm{AX}$ simulation is comparatively strong at early times $\left(>100 \mathrm{~K} \mathrm{~h}^{-1}\right)$ before settling down once the peak intensity has been achieved. In the 3-D simulation, the averaged heating rate gradually increases with the intensity. Of course, the local maximum value of the heating rate in the 3-D simulation greatly exceeds the maximum of the corresponding mean heating rate, but the local maximum exceeds also the maximum value in the AX model. The local maximum heating rates in the 3-D simulation are associated with highly localized convective cells whose areas occupy only a small fraction of the annular ring of strong heating (Fig. 5).

One might inquire now whether the differences in the heating rates between the two models suggest a relationship between the maximum mean heating rate and the instantaneous intensity of the simulated storms. Given that the heating rate is an approximate measure of the convective mass flux, one might naively suppose that the strength of the system-scale overturning circulation is related to the heating rate. A larger heating rate would thus imply a stronger overturning circulation and a greater likelihood for a stronger swirling circulation via the conventional spin-up mechanism as discussed in the Introduction. For example, one might ask whether the temporary maximum in the mean heating rate at $80 \mathrm{~h}$ in the 3D3k simulation (Fig. 6) is associated with a period of enhanced mean tangential wind speed (Fig. 1)? To explore this question we show in Fig. 7 a scatter plot of the maximum mean heating rate against the instantaneous vortex intensity. We see that although there is some scatter, the mean heating rate data comprise a cluster that suggest a monotonic, but non-linear relationship between mean heating rate and intensity. Although the AX simulation exhibits more scatter in this plot (especially early in the intensification period, when the mean intensity is low), the AX simulation follows a similar non-linear relationship. However, for the same intensity, the AX simulation has a larger maximum heating rate than the 3-D simulation. Of course, the foregoing ideas are overly simplistic because one may expect a time delay between an increase in the strength of the secondary circulation and the increase of the maximum tangential winds. This concern motivates a shift in focus to an examination of the link between the heating rate, its spatial distribution, and the rate of spinup.

As noted earlier, it is near the $66 \mathrm{~h}$ time (within the early spin-up period) when the rate of spin-up of the mean tangential wind in the 3-D model exceeds the maximum spin-up rate of the AX model (cf. Fig. 1a, compare red and green curves). In Sect. 6 a plausible explanation will be offered to explain how the intensification rate in the 3-D model can locally surpass the maximum intensification rate in the AX model despite the smaller azimuthally averaged heating rates and updraughts in the 3-D model.

At this point, the question arises whether the foregoing differences in evolution between the 3-D and AX systems are principally due to an intrinsic difference in the system-scale heating rates and spatial gradients thereof, or because of the previously postulated idea by Bryan et al. (2010 and refs.) that the "eddies" mix momentum, buoyancy and equivalent potential temperature down the local gradient of the corresponding mean scalar field. The above results hint that the effects of "eddies" in real tropical cyclones may be subtle, but important. They hint also that the azimuthally averaged and local heating rates may be at least as important in a complete explanation of the essential differences between the two systems. As a step towards examining the link between the heating rate gradient and evolution of the system-scale vortex, the next section considers axisymmetric balance aspects of spin-up in both systems.

\section{Insights from axisymmetric balance theory}

Recall that the conventional spin-up mechanism for the system-scale circulation discussed in the Introduction can be captured approximately by axisymmetric balance dynamics in association with the aggregate latent heating rate of deep convection and the corresponding mean inflow above the boundary layer. In particular, the balance framework captures the connection between the heating rate and the spin-up of the mass field of the bulk vortex. However, the balance assumption is poor in the boundary layer and the balance theory does not capture the boundary layer spin-up mechanism discussed in the Introduction. This deficiency has ramifications for the entire vortex because the radial distribution of absolute angular momentum that develops in the boundary layer is communicated to the vortex aloft by vertical advection. Since the tangential wind tends to be supergradient in the inner-core boundary layer, air that ascends from this region contributes to the spin-up of the bulk vortex as part of an adjustment process to a new balanced state as illustrated later in Sect. 5.2 (Zhang et al., 2001; Smith and Montgomery, 2010). For the remainder of this section we neglect the unbalanced 

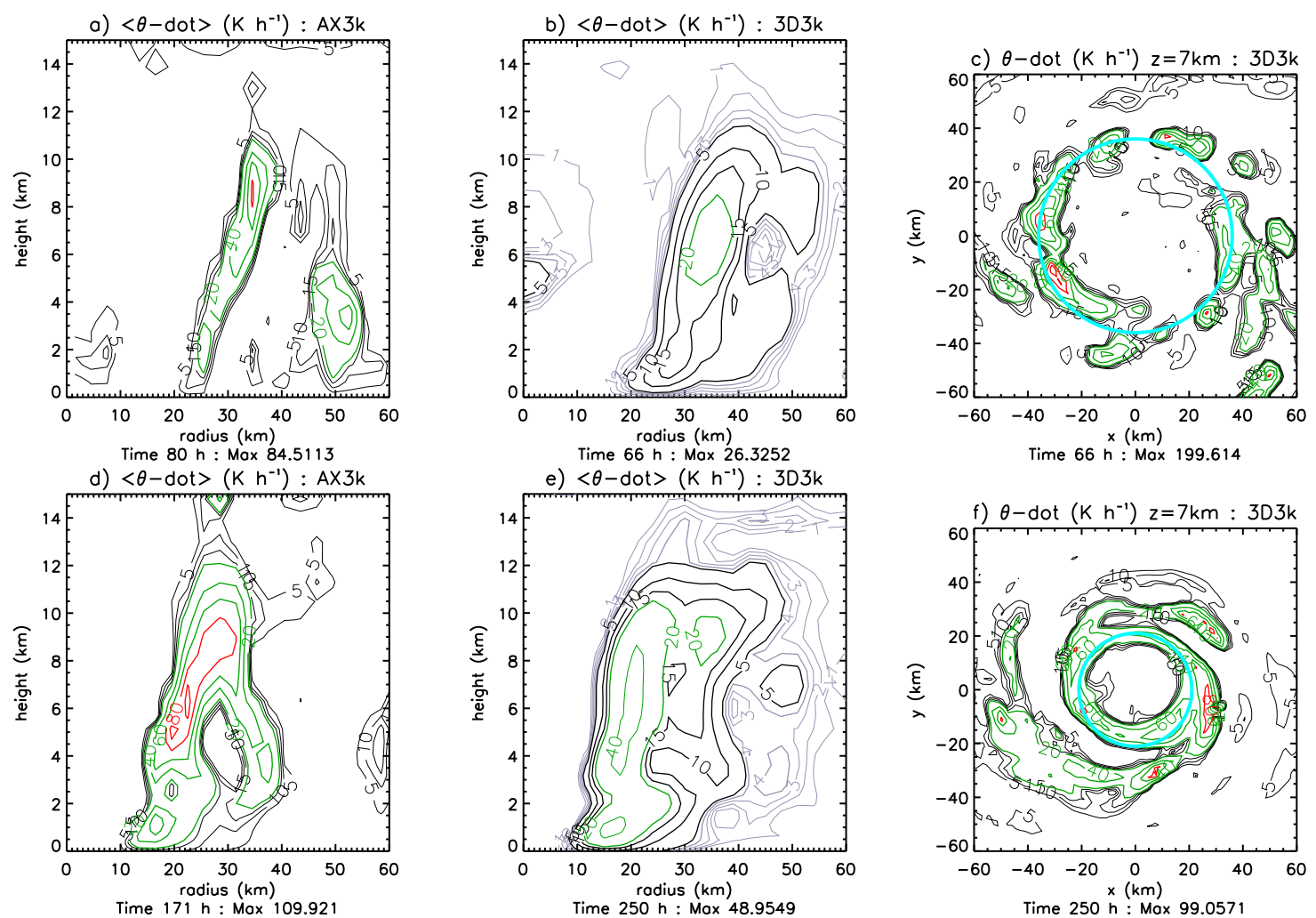

Fig. 5. Diagnosed heating rates during the periods of peak intensification and maximum quasi-steady intensity. Panels (a) and (b) are radiusheight cross sections of the azimuthal mean heating rate from the AX3k and 3D3k simulations, respectively, at a time of rapid intensification; (d) and (e) present, respectively, the same at a time of maximum intensity. Panels (c) and (f) are plan-view plots of heating rate at $z=7 \mathrm{~km}$ from the 3D3k simulation during intensification and maximum intensity; the blue circle depicts the radius of maximum azimuthal mean heating rate at this height. Thin, black contours are 5,10 , and $15 \mathrm{~K} \mathrm{~h}^{-1}$. Thick, green contours are 20,40 , and $60 \mathrm{~K} \mathrm{~h}^{-1}$. Thick, red contours are 80 and $100 \mathrm{Kh}^{-1}$.

aspects of the boundary layer and consider only the conventional spin-up mechanism.

Previous studies of the conventional spin-up process have tended to use an approach based on the Sawyer-Eliassen equation for the balanced overturning circulation forced by heat and momentum sources (Willoughby, 1979; Bui et al., 2009). Here we follow Shapiro and Montgomery (1993) and Vigh and Schubert (2009) and adopt an alternative approach based on the geopotential tendency equation. The use of the geopotential tendency equation for describing the balanced evolution of a vortex has a number of advantages over the use of the Sawyer-Eliassen equation. To begin with, unlike the Sawyer-Eliassen equation, the derivation is not degenerate for the steady state. A mathematical advantage of using the geopotential tendency equation is that it avoids the need to first invert for the overturning circulation, then advect the tangential wind component by the radial and vertical flow, and finally to link the changes in tangential wind to changes in the mass field by solving the thermal wind equation. In fact, for the idealized vortex studied by Vigh and Schubert (2009), the geopotential tendency equation gives a direct link between the heat and momentum forcing to the changes in the mass field of the vortex. Nevertheless, it remains to be shown that the geopotential tendency diagnostic is useful in more comprehensive model simulations that explicitly represent moist deep convection. On the basis of these considerations, we develop here a diagnostic tool to determine the contribution of the latent heating to the conventional spin-up mechanism in the 3-D and AX simulations and to carry out a preliminary assessment of the utility of this diagnostic framework.

\subsection{Geopotential tendency equation}

To simplify the mathematical discussion, we follow Shapiro and Montgomery (1993) and adopt an inviscid, Boussinesq formulation of the axisymmetric balance dynamics in cylindrical, pseudo-height coordinates $(r, \lambda, Z)$, where $Z$ is defined by $Z=\left(c_{\mathrm{p}} \theta_{0} / g\right)\left[1-\left(p_{\mathrm{s}} / p\right)^{k}\right], k=(\gamma-1) / \gamma, \theta_{0}$ is a reference potential temperature $(300 \mathrm{~K})$ at reference pressure $p_{\mathrm{s}}, p$ is the pressure, $g$ is the Earth's gravitational acceleration near the surface and $\gamma$ is the ratio of specific heats for dry air (Hoskins and Bretherton, 1972). A useful property of 


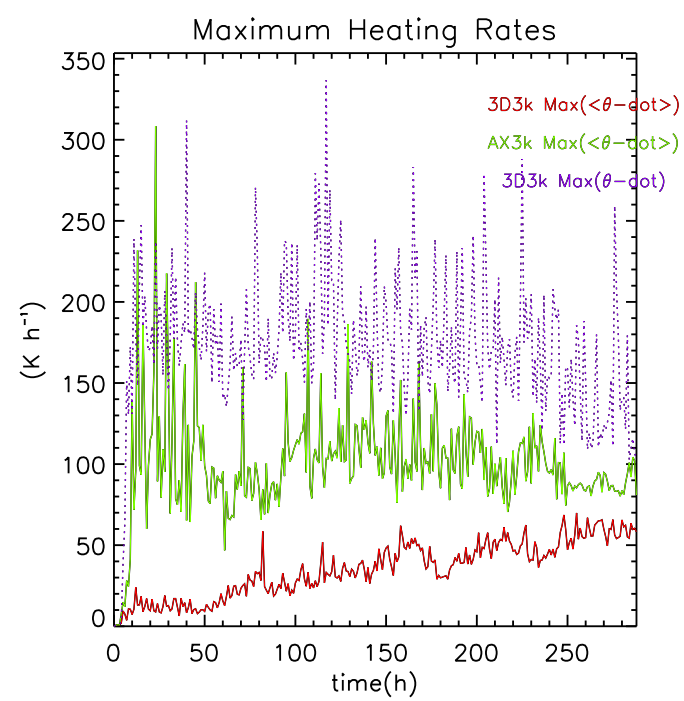

Fig. 6. Time series of maximum azimuthally averaged (mean) heating rates in the $3 \mathrm{D} 3 \mathrm{k}$ simulation (red solid curve) and the corresponding heating rate in the AX3k simulation (green solid curve). The extreme value of the heating rate found at each time in the $3 \mathrm{D} 3 \mathrm{k}$ simulation is shown by the blue-dotted curve.

this coordinate system is that $Z$ is nearly equal to height $z$ in the troposphere. For this reason, we will not distinguish between $Z$ and $z$ in the diagnostic analyses.

In the axisymmetric formulation, the axisymmetric equations of motion may be linearized about a circular vortex flow in gradient and hydrostatic balance

$f\langle v\rangle+\frac{\langle v\rangle^{2}}{r}=\frac{\partial\langle\phi\rangle}{\partial r}$

$\frac{g}{\theta_{0}}\langle\theta\rangle=\frac{\partial\langle\phi\rangle}{\partial z}$,

where $\langle v\rangle,\langle\phi\rangle,\langle\theta\rangle$ are the azimuthally averaged tangential velocity, geopotential height field and potential temperature, respectively. Here $r$ denotes radius from the circulation centre, $t$ denotes time and the azimuthal mean of some quantity $Q$, denoted by the bracket symbol, is defined as

$\langle Q\rangle(r, z, t)=\frac{1}{2 \pi} \int_{0}^{2 \pi} Q(r, \lambda, z, t) d \lambda$.

A closed evolution equation for the perturbation geopotential $\phi^{\prime}$ may be obtained from Eq. (3.10) of Shapiro and Montgomery (1993) after simplification to the limit of azimuthal

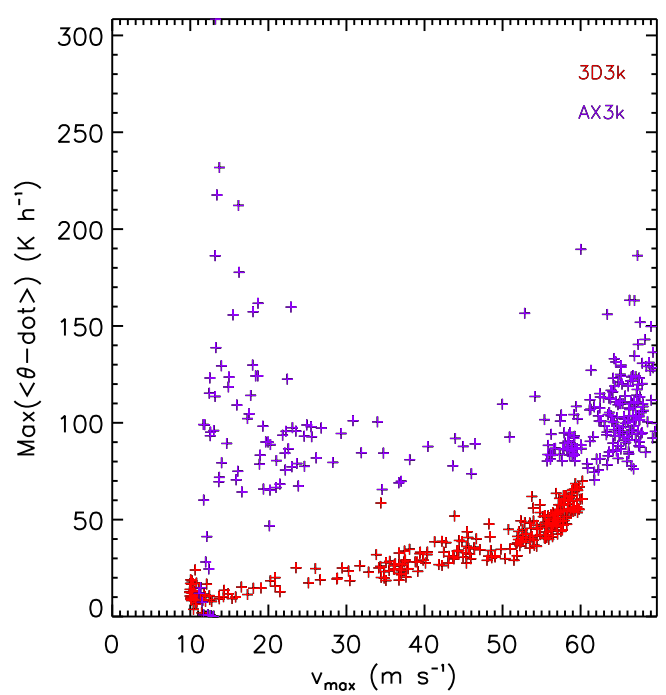

Fig. 7. The maximum azimuthally averaged heating rate from the 3D3k (red) and AX3k (blue) simulations plotted against instantaneous intensity (e.g., Fig. 1).

wavenumber zero (i.e., axisymmetric dynamics):

$$
\begin{array}{r}
\frac{\partial}{\partial t}\left\{\frac{1}{r} \frac{\partial}{\partial r}\left[\frac{r N^{2}}{\langle q\rangle\langle\xi\rangle} \frac{\partial \phi^{\prime}}{\partial r}\right]+\frac{\partial}{\partial z}\left[\frac{\langle\eta\rangle}{\langle q\rangle} \frac{\partial \phi^{\prime}}{\partial z}\right]\right. \\
\left.-\frac{1}{r} \frac{\partial}{\partial r}\left[\frac{r}{\langle q\rangle} \frac{\partial\langle v\rangle}{\partial z} \frac{\partial \phi^{\prime}}{\partial z}\right]-\frac{1}{r} \frac{\partial}{\partial z}\left[\frac{r}{\langle q\rangle} \frac{\partial\langle v\rangle}{\partial z} \frac{\partial \phi^{\prime}}{\partial r}\right]\right\} \\
=\frac{\partial}{\partial z}\left(\frac{\langle\eta\rangle}{\langle q\rangle} Q\right)-\frac{1}{r} \frac{\partial}{\partial r}\left(\frac{r}{\langle q\rangle} \frac{\partial\langle v\rangle}{\partial z} Q\right),
\end{array}
$$

where the coefficient variables in the differential operator are defined as follows: $N^{2}=\left(g / \theta_{0}\right) \partial\langle\theta\rangle / \partial z$ is the static stability of the mean vortex and environment, $\langle\eta\rangle=f+$ $1 / r \partial\langle r v\rangle / \partial r$ is the absolute vertical vorticity of the mean vortex, $\langle\xi\rangle=f+2\langle v\rangle / r$ is twice the absolute angular velocity, $Q=\left(g / \theta_{0}\right) \mathrm{D}\langle\theta\rangle / \mathrm{D} t$ is the normalized azimuthally averaged diabatic heating rate, $\mathrm{D} / \mathrm{D} t$ is the substantial derivative following the azimuthal mean circulation in $(r, z)$ coordinates,

$\langle q\rangle=\langle\eta\rangle N^{2}-\langle\xi\rangle\left(\frac{\partial\langle v\rangle}{\partial z}\right)^{2}$

is the potential vorticity (PV) of the azimuthal mean vortex in the Boussinesq approximation and $t$ is the time. Here the variable $\phi^{\prime}$ denotes the incremental change in the azimuthal mean geopotential forced by latent heating associated with the aggregate of deep convection over a small time interval. (For simplicity, we have neglected explicit "eddy" contributions associated with rectified eddy momentum and heat fluxes as represented by Sect. 5 of Shapiro and Montgomery, 1993 or McWilliams et al., 2003.) After some rearrangement, 
the right-hand-side forcing term can be rewritten as follows:

$\mathcal{L}\left(\frac{\partial \phi}{\partial t}\right)=\eta \cdot \nabla\left(\frac{Q}{\langle q\rangle}\right) \equiv S$,

where $\mathcal{L}$ is the $r-z$ differential operator acting on the geopotential tendency of the foregoing equation and $\eta$ denotes the azimuthally averaged absolute vorticity vector.

As shown by Shapiro and Montgomery (1993; their Eq. 4.10), the geopotential tendency equation is a compact form of the balanced perturbation potential vorticity equation in which the radial and vertical advection of the basic state PV is implicit. Apart from the Boussinesq approximation in pseudo-height coordinates, this geopotential tendency equation is essentially the same as that derived by Vigh and Schubert (2009; their Eqs. 2.20 and 2.21). Vigh and Schubert (2009) refer to the right-hand side of the geopotential tendency equation as the "cyclogenesis function". Since observations show clearly that tropical cyclogenesis is not an axisymmetric process (e.g., Dunkerton et al., 2009; Montgomery et al., 2012), we believe a more appropriate term for this quantity is the "spin-up function" $(S)$. For the remainder of this paper we will adhere to this latter terminology.

\subsection{Deductions from the spin-up function}

In the absence of frictional effects (focusing the discussion here on the conventional spin-up mechanism), it is clear that there will be no new generation of PV in the interior of the vortex by condensation heating (and hence no change to the mass field of the bulk vortex) unless the gradient of the mean heating rate divided by the mean PV projects non-trivially onto the absolute vorticity vector in the radius-height plane. Taking the ocean surface and tropopause to be approximately isothermal boundaries, the boundary conditions on the top and bottom of the vortex require a vanishing vertical derivative of the geopotential tendency, i.e., $\partial \phi_{t} / \partial z=0$, where $\phi_{t}$ denotes short hand for the time tendency of the mean geopotential perturbation as defined above, $\partial \phi^{\prime} / \partial t$.

Symmetry at the axis of rotation implies that $\partial \phi_{t} / \partial r=0$ at $r=0$. Boundedness of the solution requires that $\phi_{t} \rightarrow 0$ at large $r$. If the discriminant of the mean vortex is everywhere positive (i.e., $\langle\xi\rangle\langle q\rangle>0$ ), and if the spin-up function vanishes throughout the domain, we conclude from the geopotential tendency equation, with the aid of the boundary conditions discussed above, that $\phi_{t}=0$ everywhere and the flow is in a steady state. This is the "non-acceleration theorem" for inviscid, axisymmetric balance vortex dynamics (cf. McWilliams et al., 2003).

If, for the sake of argument, during the early stage of intensification, the mean vortex is approximately barotropic, the largest term in the spin-up function $S=\eta \cdot \nabla(\langle Q\rangle /\langle q\rangle)$ results from the vertical gradient of $\langle Q\rangle$. In this situation, assuming that deep convection has a simple heating profile with a maximum in the middle troposphere (à la Gill 1982), the magnitude of the mean heating rate (and its location with respect to the maximum mean vertical vorticity) controls principally the intensification rate (see Vigh and Schubert (2009)'s Eq. (2.25) and accompanying discussion). However, as the hurricane reaches maturity and the baroclinicity of the mean vortex becomes significant, the mean vorticity vector $(-\langle\partial\langle v\rangle / \partial z\rangle, 0, f+\langle\zeta\rangle)$ rotates clockwise in the $r-z$ plane and becomes locally perpendicular to the vector gradient of $(\langle Q\rangle /\langle q\rangle)$. The result is that the spin-up function approaches zero and the balanced flow tends towards a steady state ${ }^{9}$.

In general, the intensification rate in the axisymmetric balance model is controlled by the structure of the spin-up function and its radial distribution relative to the vorticity distribution (Vigh and Schubert, 2009). On the basis of the foregoing discussion, in lieu of a full inversion of the geopotential tendency equation (as employed for the Sawyer-Eliassen equation by Bui et al. (2009) or using coarse resolution data with a cumulus parameterization derived from the Geophysical Fluid Dynamics Laboratory Hurricane model by Möller and Shapiro, 2002), we will use a bulk measure of the spinup function in order to assess the balanced contribution of the mean heating rate to the change in the mass field in the 3-D and AX simulations.

In preparation for the analysis foreshadowed above, we show in Fig. 8 radius-height cross sections of selected azimuthally averaged quantities averaged between 65 and $69 \mathrm{~h}$ during the rapid intensification of the $3 \mathrm{D} 3 \mathrm{k}$ simulation. These quantities include: the azimuthal mean gradient wind

\footnotetext{
${ }^{9}$ The vanishing of the spin-up function provides a constraint on the secondary circulation in terms of the primary circulation and the diabatic heating (and, in general, other forcing terms). Although the requirement of continuity imposes a further constraint, this together with the vanishing of the spin-up function does not provide a means to determine the steady-state primary circulation and mass field. Indeed, it is unclear whether a global steady-state solution exists (Smith et al., 2012, Sect. 5.2). In the absence of friction and/or eddy fluxes of tangential momentum and moist entropy, the steady tangential momentum and moist-entropy equations imply that the azimuthally averaged secondary circulation above the boundary layer must be along both absolute angular momentum surfaces and moist isentropic surfaces. In other words, these two sets of surfaces must be congruent. Moreover, in the subsiding branch of the overturning circulation, the absolute angular momentum surfaces must have a negative radial gradient, implying that the flow there is inertially unstable and therefore no longer steady and invertible. Notwithstanding this local region of instability, the tangential flow at large radius in the outflow must become anticyclonic. Since cyclonic angular momentum is continuously removed at the surface by friction, a global steady state would require that the angular momentum of the vortex be replenished at the same rate. If sufficient angular momentum is not replenished at the lateral boundary by some means, the upper anticyclone must extend to the surface where cyclonic angular momentum would be diffused into the system. While it might be argued that some replenishment could occur as a result of horizontal eddy fluxes at the lateral boundary, it would be fortuitous if these fluxes were just sufficient to maintain a global steady state.
} 
$\left(\left\langle v_{\mathrm{g}}\right\rangle\right.$, panel a) defined by

$\left\langle v_{\mathrm{g}}\right\rangle=-\frac{f r}{2}\left(1-\sqrt{1+\frac{4}{\langle\rho\rangle f^{2} r} \frac{\partial\langle p\rangle}{\partial r}}\right) ;$

azimuthal mean potential vorticity (panel b),

$\left\langle q_{\mathrm{g}}\right\rangle=\langle\eta\rangle N^{2}-\langle\xi\rangle\left(\frac{\partial\left\langle v_{\mathrm{g}}\right\rangle}{\partial z}\right)^{2}$,

where $\langle\rho\rangle$ and $\langle p\rangle$ are the azimuthal mean air density and pressure, respectively, and the subscript " $\mathrm{g}$ " denotes evaluation using the azimuthal mean gradient wind; azimuthal mean diabatic heating rate $(\langle\dot{\theta}\rangle)$ divided by the mean potential vorticity $\left(\left\langle q_{\mathrm{g}}\right\rangle\right)$ (panel c); the radial and vertical derivative thereof (panels $\mathrm{d}$ and $\mathrm{e}$, respectively); and finally, the spin-up function $S=\zeta \cdot \nabla\left(\langle Q\rangle /\left\langle q_{\mathrm{g}}\right\rangle\right)$ (panel f).

An examination of panels (d) and (e) shows that the spinup function is dominated by the radial derivative term (panel d). The radius of maximum gradient wind is slightly larger than the time mean of $\langle v\rangle$ between 65 and $69 \mathrm{~h}$ and the maximum of $\left\langle v_{\mathrm{g}}\right\rangle$ is a little smaller than that of $\langle v\rangle\left(26 \mathrm{~m} \mathrm{~s}^{-1}\right.$ compared with $29 \mathrm{~m} \mathrm{~s}^{-1}$ in Fig. 1a).

The $\left\langle q_{\mathrm{g}}\right\rangle$-field (panel b of Fig. 8) shows a weak ring-like structure with a maximum at a finite radius just inside the RMW, the strength decreasing rapidly with radius outside the eyewall. The mean diabatic heating rate at this time (see Fig. 5b), shows two column-like structures, the innermost and strongest marking the developing eyewall. (Note that the radial domain of mean diabatic heating rate $\langle\dot{\theta}\rangle$ shown in Fig. 5 is a little more than half of the domain shown in Fig. 8.) As a result of $\left\langle q_{\mathrm{g}}\right\rangle$ becoming small outside the eyewall, the structures of $\langle\dot{\theta}\rangle$ there are accentuated in the $\langle\dot{\theta}\rangle /\left\langle q_{\mathrm{g}}\right\rangle$ field (Fig. 8c).

The radial gradient of $\langle\dot{\theta}\rangle /\left\langle q_{\mathrm{g}}\right\rangle$ (panel d), exhibits strong dipole structures on the periphery of the features in panel (c).

Figure $8 \mathrm{f}$ shows the spin-up function, $S$. Of interest is the coherent region of positive values extending vertically in the main eyewall region in association with the peak eyewall heating rate near $7 \mathrm{~km}$ altitude. This region is unlike that found in the rest of the mean vortex, which is dominated by larger-in-amplitude, but smaller-in-scale positive and negative dipole structures. However, as far as axisymmetric balanced spin-up of the inner-core tangential winds is concerned, the small-scale positive-negative dipoles in $\mathrm{S}$ would tend to be filtered in an inversion of the geopotential tendency equation (Eq. 4) on scales less than the local axisymmetric Rossby radius of deformation $L_{\mathrm{R}}=N H / \sqrt{\langle\eta\rangle\langle\xi\rangle}$ (Shapiro and Montgomery, 1993), where $N$ denotes the static stability, $H$ denotes the vertical scale of a particular dipole feature and $\langle\eta\rangle\langle\xi\rangle$ is the local centrifugal (inertial) stability parameter of the azimuthally averaged swirling flow. The local Rossby radius is roughly $60 \mathrm{~km}$ in the developing eyewall. Since the local Rossby radius of the coarse-grained flow tends to increase with radius outside the eyewall region, we would expect the small-scale positive-negative dipoles in $S$ to make a negligible contribution to the spin-up of the inner-core winds. It is for these reasons that we now focus our attention primarily on the properties of the spin-up function near and interior to the developing eyewall region.

A layer-averaging and volume-averaging of the spin-up function is computed and compared to similar properties of the gradient wind intensification rate. We define the layer average of some axisymmetric quantity $\langle Q(r, z, t)\rangle$ by

$\bar{Q}(r, t) \equiv \frac{\int_{z_{0}}^{z_{1}}\langle Q\rangle\langle\rho\rangle \mathrm{d} z}{\int_{z_{0}}^{z_{1}}\langle\rho\rangle \mathrm{d} z}$

with bounding heights $z_{0}=1.5 \mathrm{~km}, z_{1}=12 \mathrm{~km}$ and air density $\rho$. The volume average is defined by

$\hat{Q}(t) \equiv \frac{\int_{0}^{\mathrm{R}} \int_{z_{0}}^{z_{1}}\langle Q\rangle r\langle\rho\rangle \mathrm{d} z \mathrm{~d} r}{\int_{0}^{\mathrm{R}} \int_{z_{0}}^{z_{1}} r\langle\rho\rangle \mathrm{d} z \mathrm{~d} r}$,

where the outer limit of the integrating cylinder $R(t)$ is the radius of maximum $\bar{v}_{\mathrm{g}}$.

Figure 9a shows a radius-time plot of layer-averaged spinup function, $\bar{S}$, for the simulation 3D3k and Fig. 9b shows time series of the volume-averaged spin-up function, $\hat{S}$, (dotted) and volume-averaged gradient wind tendency (solid). The layer average used here comprises a height spanning the bulk of the troposphere $(1.5<z<12 \mathrm{~km})$, but purposefully omits the frictional boundary layer, the adjacent transition region $(0<z<1.5 \mathrm{~km})$, and the outflow layer $(z>12 \mathrm{~km})$ of the developing storm, where axisymmetric balance dynamics formally breaks down. Before $50 \mathrm{~h}$, the layer-averaged spin-up function displays little coherent structure ${ }^{10}$. This is broadly consistent with panel (b), which shows little tendency for spin-up of the volume-averaged gradient wind until a little after $50 \mathrm{~h}$. Subsequently, the layer-averaged spinup function is mostly positive inside the radius of maximum gradient wind. An extended period of intensification from 50 to $80 \mathrm{~h}$ corresponds to the first prolonged period of positive spin-up function. A short period of bulk weakening at $90 \mathrm{~h}$ corresponds to a pronounced minimum in spin-up function. A second prolonged intensification period shows a period where the spin-up function has large positive values. Within this period, short weakening episodes are commonly found with relatively small values of spin-up function, or alternately the RMW being unfavourably placed in relation to the peak radius of spin-up function. In the mature period (after $180 \mathrm{~h}$ ) the intensity does not change systematically,

\footnotetext{
${ }^{10} \mathrm{~A}$ Gaussian time filter is applied for the data shown and the filter is defined as follows. For a quantity $Q(r, z, t)$ and for time $t_{1} \leq t \leq t_{2}$, the smoothed quantity $Q_{\mathrm{S}}(r, z, t)$ is found over a timescale $t_{G}(=5 \mathrm{~h})$ by $Q_{\mathrm{s}}(r, z, t)=\int_{t_{1}}^{t_{2}} Q(r, z, t) G\left(t, t^{\prime} ; t_{G}\right) \mathrm{d} t^{\prime} / \int_{t_{1}}^{t_{2}} G\left(t, t^{\prime} ; t_{G}\right) \mathrm{d} t^{\prime}$ where $G\left(t, t^{\prime} ; t_{G}\right)=\exp \left(\frac{-\left(t-t^{\prime}\right)^{2}}{\left(t_{G} / 2\right)^{2}}\right)$.
} 

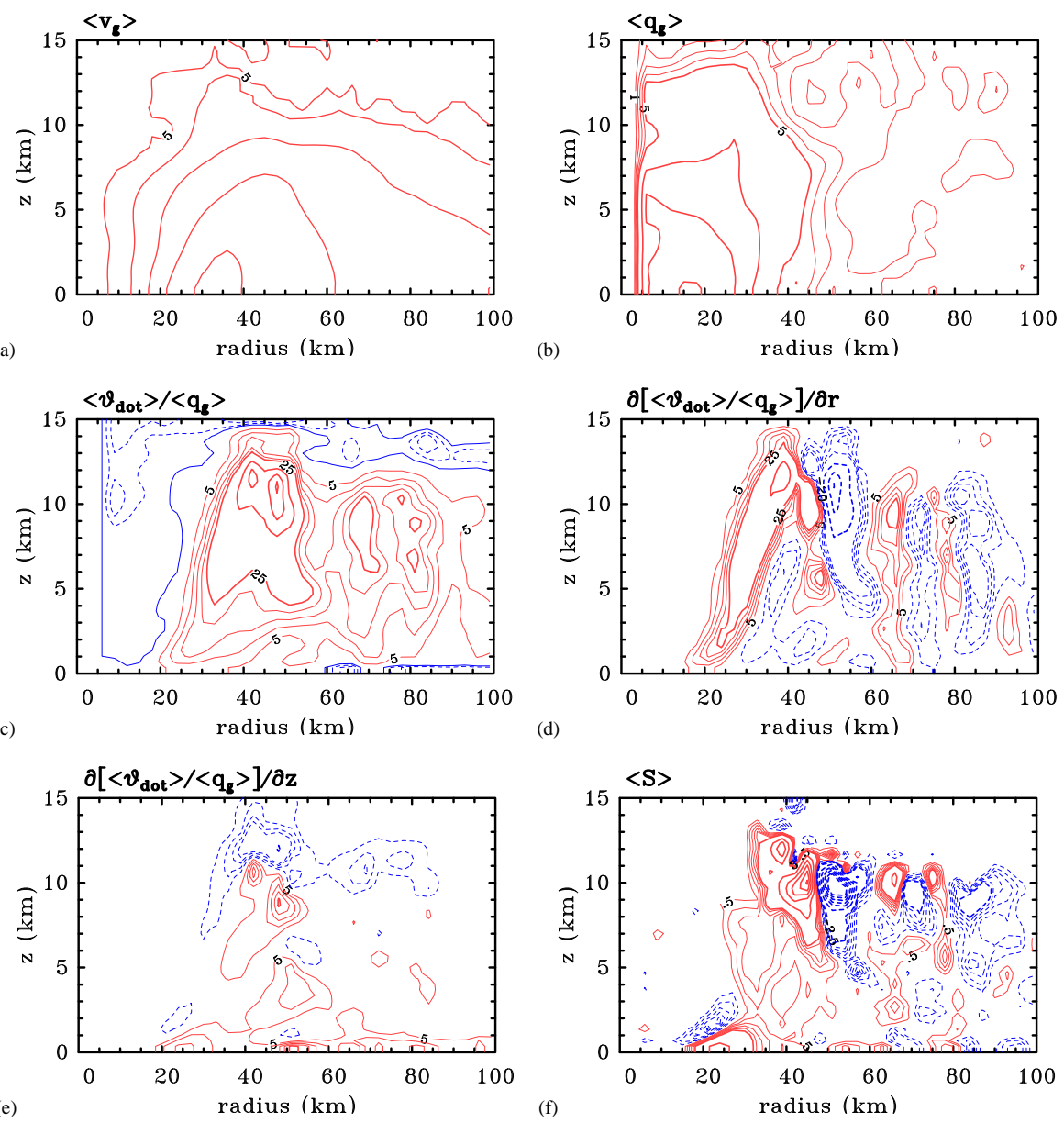

Fig. 8. Terms used in computing the spin-up function $S$ for the simulation 3D3k at a time of rapid intensification (average between 65 and $69 \mathrm{~h}$ ) with red, solid contours denoting positive values and blue dashed contours denoting negative values. (a) The azimuthal mean gradient wind, $\left\langle v_{\mathrm{g}}\right\rangle$ (Contour interval $5 \mathrm{~m} \mathrm{~s}^{-1}$ ). (b) Azimuthal mean (Boussinesq) potential vorticity, $\left\langle q_{\mathrm{g}}\right\rangle$, where the subscript "g" denotes evaluation using the gradient wind (Contour interval: thin contours $\{1,2,3,4\} \times 10^{-7} \mathrm{~s}^{-3}$; thick contours $\{5,10,15,20\} \times 10^{-7} \mathrm{~s}^{-3}$ ). (c) Azimuthal mean diabatic heating rate divided by mean potential vorticity, i.e., $\langle\dot{\theta}\rangle /\left\langle q_{\mathrm{g}}\right\rangle$. (Contour interval: blue dashed contours $\{-2,-1\} \times 10^{-3} \mathrm{~K}$ $\mathrm{s}^{2}$; thin red contours $\{5,10,15,20\} \times 10^{-3} \mathrm{~K} \mathrm{~s}^{2}$; thick red contours $\{25,50,75,100\} \times 10^{-3} \mathrm{~K} \mathrm{~s}^{2}$ ). (d) Radial derivative of $\langle\dot{\theta}\rangle /\left\langle q_{\mathrm{g}}\right\rangle$ (Contour interval: thin contours go from -2.5 to 2.5 in steps of $0.5 \mathrm{~K} \mathrm{~s}^{2} \mathrm{~m}^{-1}$; thick contours $\pm\{25,75,125, \ldots\} \mathrm{K} \mathrm{s}^{2} \mathrm{~m}^{-1}$ ). (e) Vertical derivative of $\langle\dot{\theta}\rangle /\left\langle q_{\mathrm{g}}\right\rangle$ (Contour interval: thin contours go from -2.5 to 2.5 in steps of $0.5 \mathrm{~K} \mathrm{~s}^{2} \mathrm{~m}^{-1}$; thick contours $\pm\{25,75,125, \ldots\} \mathrm{K}$ $\mathrm{s}^{2} \mathrm{~m}^{-1}$ ). (f) Spin-up function $S$, with the restriction that $\left\langle q_{\mathrm{g}}\right\rangle \geq \max \left\langle q_{\mathrm{g}}\right\rangle / 100$ (Contour intervals: thin $0.5 \times 10^{-5}$ up to $\pm 4.5 \times 10^{-5}$; thick $\left.\pm\{3,8,13, \ldots\} \times 10^{-5} \mathrm{~s}^{-1}\right)$.

yet the spin-up function remains slightly positive. This behaviour is consistent with the general increase of the maximum azimuthally averaged tangential winds shown in Fig. 1.

Figure $9 \mathrm{c}$ and $\mathrm{d}$ shows the the corresponding diagrams for the axisymmetric simulation AX3k. The time series for the RMW shows a non-systematic evolutionary behaviour for the first $80 \mathrm{~h}$ of integration with an erratic inward/outward movement evident around $30 \mathrm{~h}$ and $70 \mathrm{~h}$, followed by a systematic inward contraction beginning at about $75 \mathrm{~h}$ until about $120 \mathrm{~h}$. The second interval of RMW contraction and subsequent expansion between $55 \mathrm{~h}$ and $70 \mathrm{~h}$ suggests some similarity to an eyewall replacement cycle. However, inspection of radius- time diagrams of the vertical motion and radial inflow fields (not shown) demonstrates that this is not the case. The behaviour is rather a reflection of the chaotic nature of the (ringlike) convection which is highly disorganized at early times in the simulation.

The spin-up function in the AX simulation is generally less coherent with time than that found in 3D3k, showing also large negative as well as positive values near and away from the RMW. The principal spin-up episode (80 to $100 \mathrm{~h}$ ) does exhibit a persistently positive signature near and just inside the RMW (with the AX spin-up function approximately twice as large as the corresponding spin-up function in the 


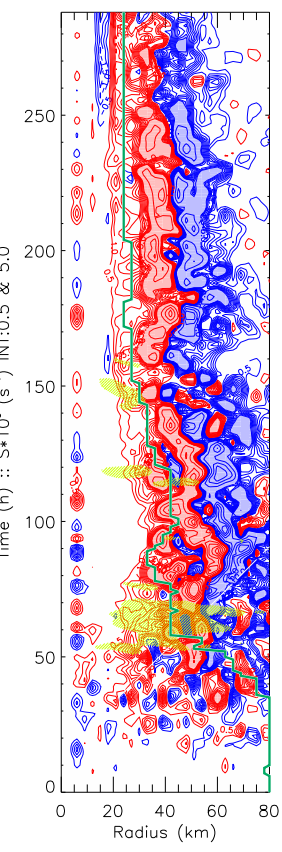

$(a, b)$

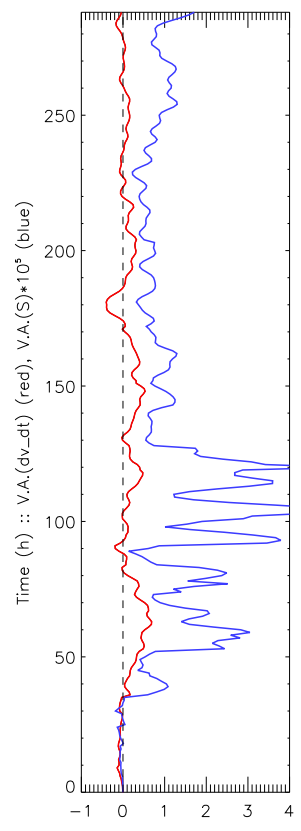

$(\mathrm{c}, \mathrm{d})$
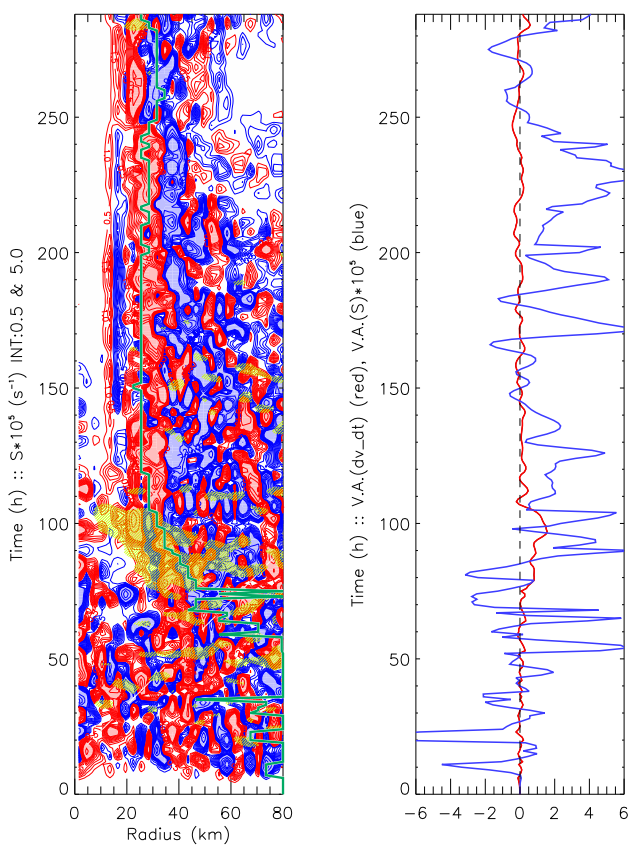

Fig. 9. (a) Radius-time plot of layer-averaged spin-up function, $\bar{S}$ for the simulation 3D3k. Red contours denote positive values of $\bar{S}$, blue contours denote negative values. Thin contour interval $1 \times 10^{-5} \mathrm{~s}^{-1}$ up to magnitude $4 \times 10^{-5} \mathrm{~s}^{-1}$; thick contour interval $5 \times 10^{-5} \mathrm{~s}^{-1}$. Zero contour is omitted. Yellow shading denotes regions where layer-averaged intensification rate for the gradient wind exceeds $\partial \bar{v}_{\mathrm{g}} / \partial t>0.5$ $\mathrm{m} \mathrm{s}^{-1} \mathrm{~h}^{-1}$; thick green curve denotes the radius of maximum $\bar{v}_{\mathrm{g}}$. For reasons given in the text, the layer average used comprises a height spanning the bulk of the troposphere $(1.5<z<12 \mathrm{~km})$, but purposefully omits the frictional boundary layer $(0<z<1.5 \mathrm{~km})$ and outflow layer $(z>12 \mathrm{~km})$ of the developing storm where axisymmetric balance dynamics formally breaks down. (b) Time series of the volumeaveraged spin-up function $\hat{S}$ in units of $1 \times 10^{-5}\left(\mathrm{~s}^{-1}\right)$ (blue) and volume-averaged gradient wind tendency (in units of $\mathrm{m} \mathrm{s}^{-1} \mathrm{~h}^{-1}$ ) (red). The volume averaging is carried out from the axis of rotation to the radius of maximum layer-averaged gradient wind (thick green curve in panel a) and over the same height as employed in panel (a). Panels (c) and (d) are the corresponding plots for the axisymmetric simulation AX3k. The contour intervals are identical. All fields have a Gaussian time smoother applied prior to layer averaging.

3-D simulation during its first period of rapid spin-up (50$80 \mathrm{~h})$ ). However, small-scale positive and negative values of the spin-up function extend radially inwards and outwards of the RMW and the most extended period of positive values of spin-up function (190 to $240 \mathrm{~h}$ ) corresponds to a long, slow weakening period of the AX simulation (cf. Fig. 1). From the perspective of balance dynamics, these findings suggest a qualitative and quantitative difference between the AX and 3-D intensification process.

We turn now to consider more general aspects of spinup, including the unbalanced aspects thereof. In particular, we determine the quantitative contribution of "mean" and "eddy" processes in the tangential momentum equation for the mean vortex.

\section{Tangential momentum equation}

This section presents an analysis of the azimuthally averaged tangential momentum equation, which serves as a first step in understanding the role of "mean" and "eddy" processes in the evolution of the mean vortex in the 3D3k simulation.
This analysis will be compared with a similar one for the corresponding AX3k simulation ${ }^{11}$.

In cylindrical-polar coordinates, the tangential momentum equation in the CM1 model has the form

$$
\begin{aligned}
\frac{\partial v}{\partial t}= & -u \frac{\partial v}{\partial r}-\frac{v}{r} \frac{\partial v}{\partial \lambda}-w \frac{\partial v}{\partial z}-\left(f+\frac{v}{r}\right) u- \\
& \frac{c_{\mathrm{p}} \theta_{\rho}}{r} \frac{\partial \pi}{\partial \lambda}+D_{v},
\end{aligned}
$$

where $(u, v, w)$ are the velocity components in the cylindrical coordinate system $(r, \lambda, z)$ and $D_{v}$ is the subgrid-

\footnotetext{
${ }^{11}$ Although, as discussed in the Introduction, the absolute angular momentum (circulation) equation provides a useful framework for interpreting certain features of vortex evolution, we prefer to use the tangential momentum equation here because the model is formulated to solve Newton's second law of motion and horizontal and vertical momentum are the diffused quantities on the subgrid scale. While the two equations are physically equivalent, the tangential momentum equation has advantages when discussing subgrid scale processes. For example, down-gradient transfer of tangential momentum is not necessarily equivalent to the down-gradient diffusion of absolute angular momentum.
} 
scale forcing of $v$ (including diffusive tendencies in the interior flow and the boundary layer). In the diagnosis of the 3D3k experiment, the origin of the cylindrical coordinate system is defined as the minimum of the time-averaged surface pressure. The averaging interval for the surface pressure field on the high resolution subdomain is the nominal time plus/minus $3 \mathrm{~h}$.

Applying the azimuthal average operator to the tangential velocity Eq. (11), the equation for the azimuthally averaged tangential wind tendency is

$$
\begin{aligned}
\frac{\partial\langle v\rangle}{\partial t}= & \underbrace{-\langle u\rangle\langle f+\zeta\rangle}_{V_{\mathrm{m} \zeta}}-\underbrace{\langle w\rangle \frac{\partial\langle v\rangle}{\partial z}}_{V_{\mathrm{mv}}} \underbrace{-\left\langle u^{\prime} \zeta^{\prime}\right\rangle}_{V_{\mathrm{e} \zeta}} \underbrace{\left\langle w^{\prime} \frac{\partial v^{\prime}}{\partial z}\right\rangle}_{V_{\mathrm{ev}}} \\
& +\underbrace{c_{\mathrm{p}}\left\langle\frac{\theta_{\rho}^{\prime}}{r} \frac{\partial \pi^{\prime}}{\partial \lambda}\right\rangle}_{V_{\mathrm{ppg}}}+\underbrace{\left\langle D_{v}\right\rangle}_{V_{\mathrm{d}}} .
\end{aligned}
$$

Here and elsewhere, the prime (or "eddy") denotes a departure from the azimuthal mean. The vertical component of relative vorticity is denoted by $\zeta$. The terms on the right hand side are, respectively, the mean radial influx of absolute vertical vorticity $\left(V_{\mathrm{m} \zeta}\right)$, the mean vertical advection of mean tangential momentum $\left(V_{\mathrm{mv}}\right)$, the eddy radial vorticity flux $\left(V_{\mathrm{e} \zeta}\right)$, the vertical advection of eddy tangential momentum $\left(V_{\mathrm{ev}}\right)$, the azimuthal perturbation pressure gradient force per unit mass $\left(V_{\mathrm{ppg}}\right)$, and the combined diffusive and planetary boundary layer tendency $\left(V_{\mathrm{d}}\right)$. This methodology represents the traditional Eulerian approach to "eddy-mean" partitioning in the tangential wind equation (e.g., Hendricks et al., 2004; Montgomery et al., 2006; Yang et al., 2007). Although we do not depart from this approach here, it should be pointed out that, in principle, highly localized asymmetric features can project upon what are termed here as "mean" terms. For example, if we suppose an otherwise axisymmetric vortex with an imposed, single, large-amplitude, positive anomaly in vertical motion, this anomaly will project onto both the vertical eddy and mean terms.

To quantify the effect of the subgrid-scale processes on the vortex development, it proves convenient to separate the subgrid-scale diffusive tendency of the tangential wind component into radial $\left(V_{\mathrm{d} r}\right)$ and vertical $\left(V_{\mathrm{d} z}\right)$ contributions to the divergence of the subgrid-scale momentum fluxes

$$
\left\langle D_{v}\right\rangle=\underbrace{\frac{1}{r^{2}} \frac{\partial\left\langle r^{2} \tau_{r \lambda}\right\rangle}{\partial r}}_{V_{\mathrm{d} r}}+\underbrace{\frac{1}{\rho_{0}} \frac{\partial\left\langle\rho_{0} \tau_{\lambda z}\right\rangle}{\partial z}}_{V_{\mathrm{d} z}},
$$

where the subgrid-scale momentum fluxes are related to the mean strain-rate tensor in cylindrical coordinates by

$$
\begin{aligned}
& \left\langle\tau_{r \lambda}\right\rangle=\left\langle K_{\mathrm{m}, \mathrm{h}}\left(\frac{1}{r} \frac{\partial u}{\partial \lambda}+r \frac{\partial v / r}{\partial r}\right)\right\rangle \\
& \left\langle\tau_{\lambda z}\right\rangle=\left\langle K_{\mathrm{m}, \mathrm{v}}\left(\frac{1}{r} \frac{\partial w}{\partial \lambda}+\frac{\partial v}{\partial z}\right)\right\rangle
\end{aligned}
$$

and $\rho_{0}(z)$ is the basic state density profile.

To achieve an accurate representation of the subgrid scale diffusion in the tangential momentum budget of the 3-D model, we found it necessary first to calculate the subgrid scale diffusion of horizontal momentum on the stretched Cartesian grid and then transform to cylindrical coordinates. In brief, the diffusive tendency of horizontal momentum per unit mass $\boldsymbol{F}$ is derived from two vectors, the effect of horizontal diffusion on the horizontal wind $\boldsymbol{F}_{\mathrm{h}}=F_{x, \mathrm{~h}} \hat{x}+F_{y, \mathrm{~h}} \hat{y}$ and the effect of vertical diffusion on the horizontal wind $\boldsymbol{F}_{\mathrm{v}}=F_{x, \mathrm{v}} \hat{x}+F_{y, \mathrm{v}} \hat{y}$, where the coefficient functions are the projections onto the horizontal Cartesian coordinate unit vector directions, $\hat{x}$ and $\hat{y}$, respectively. Outside of the lowest model level, the stress tensor $\tau$ is computed using the model output diffusivities $K_{\mathrm{m}, \mathrm{h}}$ and $K_{\mathrm{m}, \mathrm{v}}$ and the proper spatial derivatives of the Cartesian velocity field $\boldsymbol{u}=u_{x} \hat{x}+u_{y} \hat{y}+w \hat{z}$ (where $\hat{z}$ is the vertical Cartesian coordinate unit vector). At the lowest model grid level $(z=25 \mathrm{~m})$, the shear stresses are computed using the model's bulk-aerodynamic formula: $\tau_{x z}=\rho_{0} C_{\mathrm{D}} u_{x} c$ and $\tau_{y z}=\rho_{0} C_{\mathrm{D}} u_{y} c$, with the near-surface wind speed denoted by $c=\sqrt{u_{x}^{2}+u_{y}^{2}}$. The $x$ and $y$ components of the diffusive tendency are obtained from $F_{x, \mathrm{~h}}=$ $\rho_{0}^{-1}\left(\frac{\partial \tau_{x x}}{\partial x}+\frac{\partial \tau_{x y}}{\partial y}\right), F_{y, \mathrm{~h}}=\rho_{0}^{-1}\left(\frac{\partial \tau_{x y}}{\partial x}+\frac{\partial \tau_{y y}}{\partial y}\right), F_{x, \mathrm{v}}=\rho_{0}^{-1} \frac{\partial \tau_{x z}}{\partial z}$, and $F_{y, \mathrm{v}}=\rho_{0}^{-1} \frac{\partial \tau_{y z}}{\partial z}$. The desired diffusive tendency $D_{v}$ for the tangential velocity $v$ is then $D_{v}=\boldsymbol{F} \cdot \hat{\lambda}=F_{y} \cos \lambda-$ $F_{x} \sin \lambda$, where $\hat{\lambda}$ is the azimuthal unit vector.

\subsection{The 3D3k simulation}

Figure 10 shows, inter alia, time averages of the three velocity components and all but one term in the azimuthally averaged tangential momentum equation during the period of rapid intensification (between 65 and $69 \mathrm{~h}$ ). The term not shown is the azimuthal average of the azimuthal perturbation pressure gradient force per unit mass (term $V_{\mathrm{ppg}}$ in Eq. 3), which is much smaller than the other terms because the azimuthal variation in virtual potential temperature is small. The main features of the azimuthal-mean vortex during this period are as follows. The radial velocity field is dominated by a shallow layer of strong inflow below $1 \mathrm{~km}$ associated with the frictional boundary layer, a deeper layer of outflow in the upper troposphere and weak inflow through the midtroposphere outside the eyewall. The maximum tangential wind component is found at low-levels, near the top of the strong inflow layer. The dominant feature of the vertical velocity component is an annular region of strong upward motion with the principal maximum in the upper troposphere at $z=10 \mathrm{~km}$, extending downward to a slight extremum at the top of boundary layer. As indicated in Fig. 5, the updraught at this time encircles much, but not all of the circulation centre. The secondary maximum of the vertical velocity near the top of the boundary layer at this time, during the second intensification period (not shown), and during the mature period 

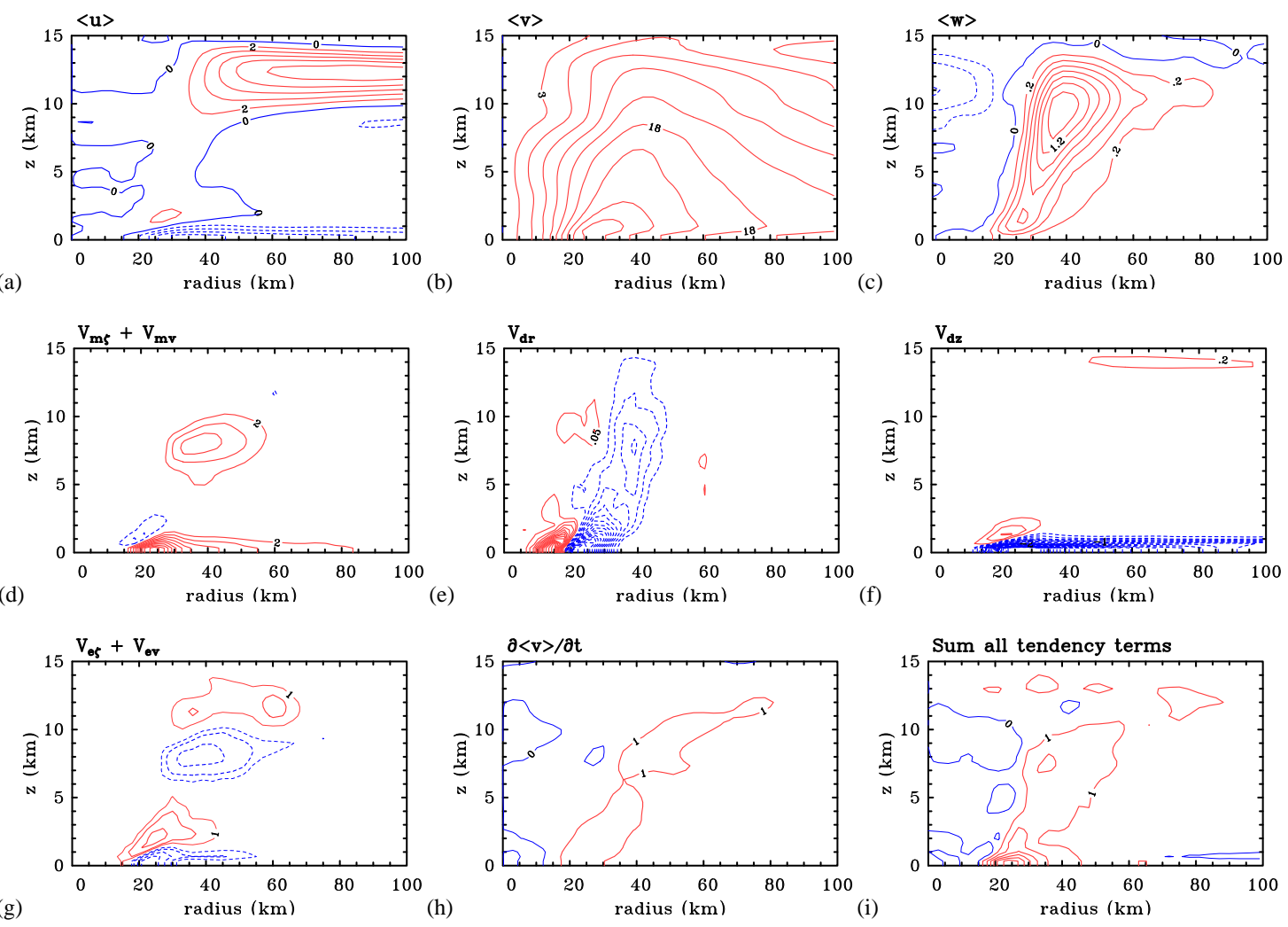

Fig. 10. Terms of the tangential wind tendency equation for the simulation 3D3k, averaged during the period of rapid intensification (65-69 h). Red contours denote positive values; blue contours denote negative values. (a) Radial velocity $\langle u\rangle$ (Contour interval $2 \mathrm{~m} \mathrm{~s}^{-1}$ ); (b) tangential velocity $\langle v\rangle$ (Contour interval $3 \mathrm{~m} \mathrm{~s}^{-1}$ ); (c) vertical velocity $\langle w\rangle$ (Contour interval $0.2 \mathrm{~m} \mathrm{~s}^{-1}$; blue contours $-0.1,-0.05$, and $0 \mathrm{~m} \mathrm{~s}^{-1}$ ); (d) sum of mean vorticity influx and vertical advection $V_{\mathrm{m} \zeta}+V_{\mathrm{mv}}$ (Contour interval $2 \mathrm{~m} \mathrm{~s}^{-1} \mathrm{~h}^{-1}$ ); (e) radial (subgrid-scale) diffusive tendency $V_{\mathrm{d} r}$ (Contour interval $\left.0.05 \mathrm{~m} \mathrm{~s}^{-1} \mathrm{~h}^{-1}\right)$; (f) vertical (subgrid-scale) diffusive tendency $V_{\mathrm{d} z}$ (Contour interval $0.2 \mathrm{~m} \mathrm{~s}^{-1} \mathrm{~h}^{-1}$ ); $(\mathrm{g})$ combined eddy tendencies $V_{\mathrm{e} \zeta}+V_{\mathrm{ev}}$ (Thick contour interval is $2 \mathrm{~m} \mathrm{~s}^{-1} \mathrm{~h}^{-1}$, thin contours are $\pm 1 \mathrm{~m} \mathrm{~s}^{-1}$ ); (h) mean tangential wind tendency $\partial\langle v\rangle / \partial t$ (Contour interval $1.0 \mathrm{~m} \mathrm{~s}^{-1} \mathrm{~h}^{-1}$ ); (i) sum of all tendency terms (Contour interval $1.0 \mathrm{~m} \mathrm{~s}^{-1} \mathrm{~h}^{-1}$ ).

(shown below) is associated with the inertial turning of the boundary layer inflow into the updraught (Smith et al., 2008, 2009). There is a small region of weak subsidence inside the main updraught annulus near the axis in the upper troposphere, an indication of a developing eye region. All of these features are similar to ones found in Zhang et al. (2001) for the specific "real case" study case of Hurricane Andrew 1992 and Smith et al. (2009) using an idealized model formulation.

We consider next the contributions to the azimuthal-mean tangential wind tendency from the sum of the mean vorticity influx and mean vertical advection shown in Fig. 10d, and the radial and vertical subgrid-scale terms shown in Fig. 10e and $\mathrm{f}$. The main contribution to the spin-up of the maximum tangential wind is associated with the import of mean cyclonic absolute vorticity in the boundary layer and its vertical advection into the eyewall updraught. This result corroborates that of Bui et al. (2009, Fig. 9a, c, g and e). The boundary layer import of absolute vorticity is largely opposed by the loss of tangential momentum to the surface by friction (compare panels $d$ and $f$ ). Note that the radial diffusion (panel e) shows a positive tendency in the eye region (particularly between $5<r<20 \mathrm{~km}$ ), implying that the eye is being spun up primarily by the downgradient diffusion of azimuthal-mean angular velocity. This mechanical spin-up of the eye is accompanied by a diffusive spin-down tendency of the maximum tangential winds (Malkus, 1958; Kurihara and Bender, 1982; Emanuel, 1997; Schubert et al., 1999). However, as shown below and in Sect. 6, the spin-up of the eye is more than simply a diffusive process.

Figure $10 \mathrm{~g}$ shows the sum of the resolved radial eddy vorticity flux and eddy vertical advection tendencies. In the upper troposphere, the eddies contribute to a positive tendency of the mean tangential wind. In the middle troposphere, the positive tendency of the mean tangential wind from the mean terms (mainly vertical advection) is partly compensated by the negative tendency from the combined eddy terms. There is some compensation also between the mean and eddy tendencies in the lower troposphere. Although not shown here, an examination of the individual eddy terms in the lower troposphere just above the boundary layer inflow indicates that 

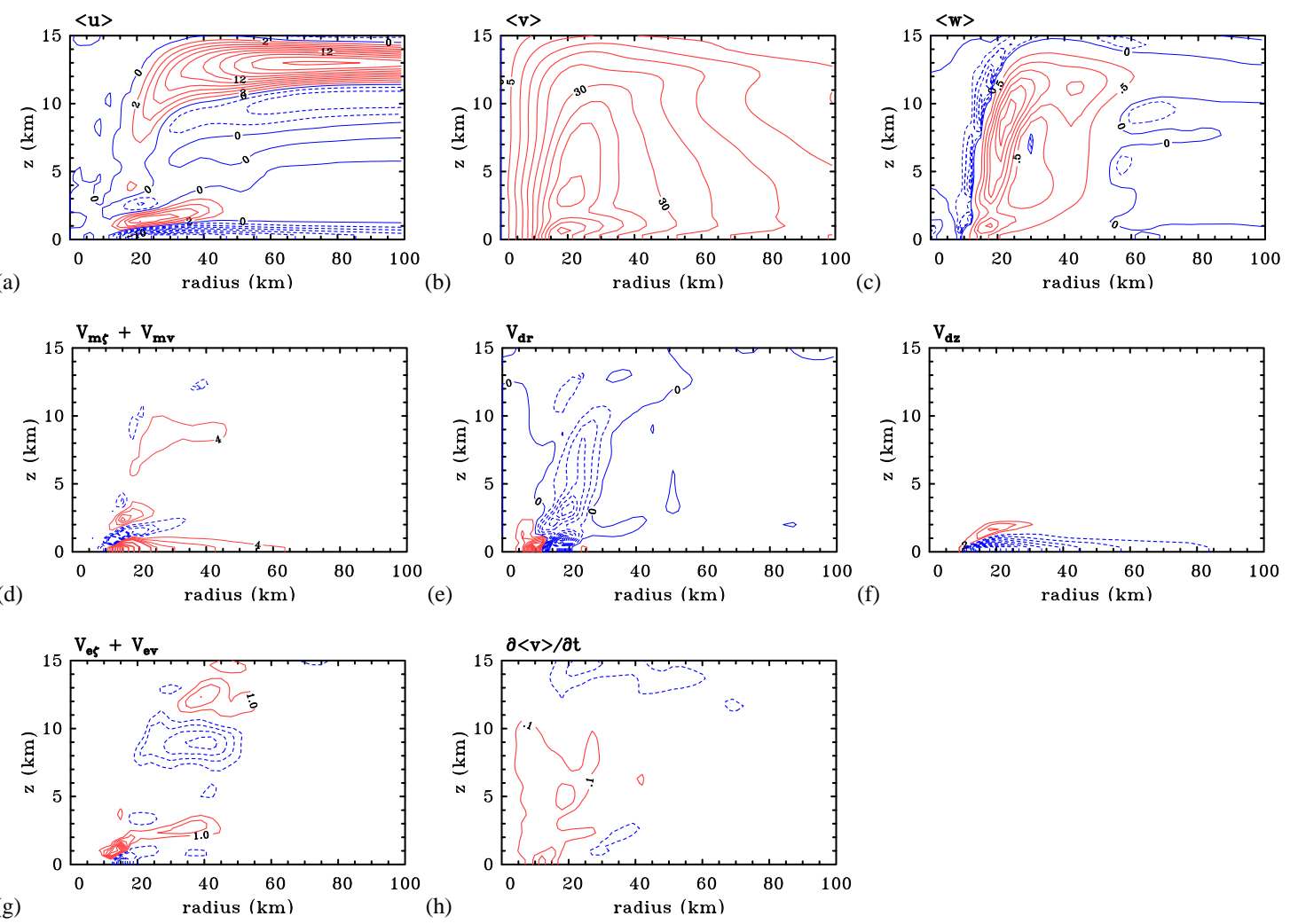

Fig. 11. Terms of the tangential wind tendency equation for the simulation 3D3k, averaged during the mature stage (214-226 h). Red contours denote positive values; blue contours denote negative values. (a) Radial velocity $\langle u\rangle$ (Contour interval $2 \mathrm{~m} \mathrm{~s}^{-1}$ ); (b) tangential velocity $\langle v\rangle$ (Contour interval $5 \mathrm{~m} \mathrm{~s}^{-1}$ ); (c) vertical velocity $\langle w\rangle$ (Contour interval for positive $0.5 \mathrm{~m} \mathrm{~s}^{-1}$; negative contours $-0.05,-0.1$, $\left.-0.15,-0.2 \mathrm{~m} \mathrm{~s}^{-1}\right)$; (d) sum of mean vorticity influx and vertical advection $V_{\mathrm{m} \zeta}+V_{\mathrm{mv}}$ (Contour interval: thin contours $4 \mathrm{~ms}^{-1} \mathrm{~h}^{-1}$, thick contours $20 \mathrm{~m} \mathrm{~s}^{-1} \mathrm{~h}^{-1}$ ); (e) radial (subgrid-scale) diffusive tendency $V_{\mathrm{d} r}$ (Contour interval $0.5 \mathrm{~m} \mathrm{~s}^{-1} \mathrm{~h}^{-1}$ ); (f) vertical (subgrid-scale) diffusive tendency $V_{\mathrm{d} z}$ (Contour interval $\left.2 \mathrm{~m} \mathrm{~s}^{-1} \mathrm{~h}^{-1}\right)$; (g) combined eddy tendencies $V_{\mathrm{e} \zeta}+V_{\mathrm{ev}}\left(\right.$ Contour interval $\left.2 \mathrm{~m} \mathrm{~s}^{-1} \mathrm{~h}^{-1}\right) ;(\mathbf{h}) \mathrm{mean}$ tangential wind tendency $\partial\langle v\rangle / \partial t$ (Contour interval $\left.0.1 \mathrm{~m} \mathrm{~s}^{-1} \mathrm{~h}^{-1}\right)$.

the horizontal eddy vorticity influx can be negative at times and positive at other times, while the vertical eddy momentum advection term is generally positive there and exceeds the horizontal contribution. (A summary of two intensification periods for $3 \mathrm{D} 3 \mathrm{k}$ and a full time series for the $1-2 \mathrm{~km}$ layer above the boundary layer inflow in shown in Sect. 6b.) While the combined mean term tends to be larger than the combined eddy term in the middle and upper troposphere, the eddies contribute almost equally in spinning up the lower troposphere above the boundary layer as the mean terms contribute to spinning down the same layer. The foregoing results may be interpreted physically to mean that there is azimuthal variability of the boundary layer inflow and outflow associated with the rotating deep convection along the eyewall.

Figure $10 \mathrm{~h}$ shows the azimuthal-mean tangential wind tendency from model output (the left-hand side of Eq. 12), while Fig. 10i shows the corresponding tendency diagnosed from the sum of mean and eddy terms plus the subgrid scale (boundary layer and diffusion) processes (the right hand side of Eq. 12). The two panels agree reasonably well quantitatively, although three sources of error in our calculations must be acknowledged. These are the sampling of the output data, the evaluation of parameterized internal diffusion and surface fluxes, and the use here of centred spatial differences to calculate advection, whereas the CM1 model uses a 5thorder upstream advective scheme. The nature of these errors is not to change the overall sign or reverse the overall direction of the gradient of these computed tendency fields, but the errors become most apparent in the boundary layer when the storm approaches a mature intensity and where both the second and third sources of error are especially prevalent. For the time interval shown in Fig. 10, the maximum tangential winds are found to reside in the eyewall region near the top of the boundary layer, where the radial spin-up mechanism associated with the sum of the vorticity influx and vertical diffusion terms is a maximum. This spin-up is conveyed up the eyewall by the combined mean terms, primarily through the vertical advection of tangential momentum. 
The foregoing diagnoses suggest that the presence of the eddies, while quantitatively non-negligible and contributing positively to the spin-up of the system-scale swirling wind above the boundary layer, does not fundamentally alter the view that the maximum tangential wind tendency occurs in the boundary layer. The results strongly support the findings of Zhang et al. (2001), Smith et al. (2009), Smith and Thomsen (2010), and Sanger et al. (2013) that the maximum tangential wind occurs within the boundary layer inflow during the spin-up process.

Figure 11 shows radius-height cross sections corresponding to those in Fig. 10 during the quasi-steady stage of the 3D3k simulation (214-222 h). (As a reminder, the quasisteady characterization is based on the time evolution of $V_{\max }$ and does not imply that the vortex as a whole is close to a steady state.) As foreshadowed above, it is of interest to note the double maximum of the mean tangential wind field. The region between these two maxima coincides with the region of strong outflow just above the low-level inflow maximum. This outflow, in conjunction with the approximate material conservation of absolute angular momentum, leads to a reduction of the tangential wind speed to the extent that the flow becomes subgradient as it ascends into the eyewall. The imbalance between the radial pressure gradient and the sum of Coriolis and centrifugal forces leads again to a shallow layer of inflow, and thereby to a secondary acceleration of the tangential wind. This "inertial recoil" effect is in essence a standing centrifugal wave, which is damped as the air ascends and the tangential wind and radial pressure gradient come into balance above the boundary layer. The pattern of inflow and outflow is similar to that during the first intensification period, but the boundary layer inflow and uppertropospheric outflow have approximately doubled in strength and the deep inflow above the boundary layer has collapsed and has been replaced by weak outflow below $6 \mathrm{~km}$, at least to a radius of $100 \mathrm{~km}$. Also, the region of inflow below the upper-tropospheric outflow layer has expanded in areal extent and strengthened. A prominent feature of the vertical velocity field is more pronounced descent through the eye than during the first intensification period. At this stage, the eyewall updraught fully encircles the centre of circulation.

The individual tendency terms exhibit a broadly similar structure to those found during the intensification period, but the net tendency is small near the RMW by definition of the quasi-steady state. However, there is a continued weak spinup of the eye during this period. Unlike during the first intensification phase, the radial diffusion has a near-zero tendency near the maximum of spin-up tendency $\partial\langle v\rangle / \partial t$, so that the spin-up of the low- to mid-tropospheric eye region includes also the combined mean and eddy tendencies.

\subsection{The AX3k simulation}

Figure 12 shows radius-height cross sections of both the vortex structure and the contributions to the tangential wind ten- dency during the period of maximum intensification (77$83 \mathrm{~h}$ ). As noted in Sect. 3.1, some of the fields are noisier than their counterpart in the azimuthally averaged fields from the 3-D model.

The maximum radial inflow near the surface occurs at about $30 \mathrm{~km}$ radius and has a magnitude comparable to that of the 3-D simulation $\left(-8.2 \mathrm{~m} \mathrm{~s}^{-1}\right.$ in AX3k versus $-9.4 \mathrm{~m} \mathrm{~s}^{-1}$ in $3 \mathrm{D} 3 \mathrm{k}$ ) during the period of maximum intensification. However, the boundary layer inflow structure shows a secondary maxima near $65 \mathrm{~km}$ radius and this maximum appears to be associated with a secondary updraught complex near $50 \mathrm{~km}$ radius. From a phenomenological viewpoint, this outer convective complex resembles a secondary eyewall, although all convection in the $\mathrm{AX}$ model has a ring-like structure and an enhanced inflow maximum driven presumably by the convection. The role of boundary-layer dynamics in the formation of secondary eyewall features has been articulated recently by Huang et al. (2012).

The flow fields in Fig. 12 show many similarities to those in the 3-D simulation, except that the eyewall updraught is narrower radially and strong tangential winds $\left(\approx 15 \mathrm{~m} \mathrm{~s}^{-1}\right)$ extend to a greater height. The contributions to the tangential wind tendency from the sum of the vorticity influx and vertical advection are shown in Fig. 12d, and the radial and vertical subgrid-scale terms are shown in Fig. 12e and f. As in the 3D3k simulation (Fig. 10), the main contribution to the spin-up of the maximum tangential wind is associated with the import of cyclonic absolute vorticity in the boundary layer, but again there is some compensation by the upward vertical advection of momentum out of the boundary layer. Once more, this positive sum is compensated to a large extent by the loss of tangential momentum to the surface by friction (compare Fig. 12d and f) and the maximum tendency (Fig. $12 \mathrm{~g}$ ) is still near the top of the boundary layer, below the eyewall updraught. Again, as in the 3D3k simulation, the swirling circulation within the developing eye is spun up by radial diffusion (Fig. 12e), but consistent with the much tighter radial gradients evident, the horizontal extent of this diffusive spin-up effect is very limited radially.

Figure 13 shows radius-height cross sections illustrating the vortex structure and contributions to the tangential wind tendency during the quasi-steady stage of the AX3k simulation (176-200 h). Once again, we see a double maximum of the mean tangential wind field and the signature of a standing centrifugal wave in the radial velocity field below $4 \mathrm{~km}$ altitude. The maximum boundary layer inflow has tripled in value relative to that found during the period of rapid intensification and the outflow maximum has approximately doubled. The maximum vertical velocity is a little over $5 \mathrm{~m} \mathrm{~s}^{-1}$ at a height of about $8 \mathrm{~km}$, but there is a secondary maximum of $2.5 \mathrm{~m} \mathrm{~s}^{-1}$ at the top of the boundary layer. This local maximum is associated with the inertial turning of the boundary layer inflow into the updraught (Smith et al., 2008, 2009). Unlike the $3 \mathrm{D} 3 \mathrm{k}$ simulation, there is only weak subsidence within the eye region during this quasi-steady period. 

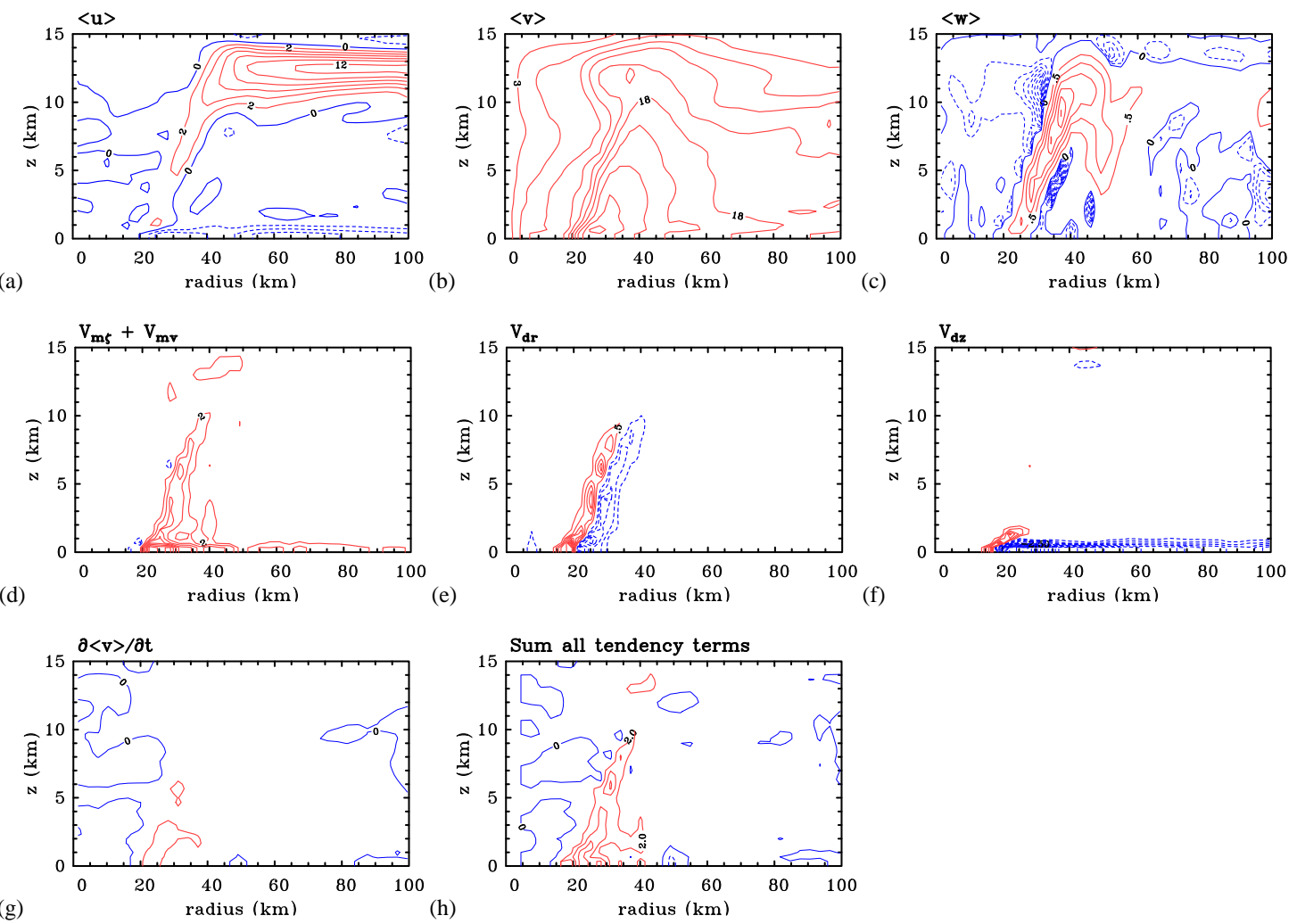

Fig. 12. Terms of the tangential wind tendency equation for the simulation AX3k, averaged during the rapid intensification stage (77$83 \mathrm{~h}$ ). Red color denotes positive values; blue color denotes negative values. (a) Radial velocity $\langle u\rangle\left(\right.$ Contour interval $2 \mathrm{~m} \mathrm{~s}^{-1}$ ); (b) tangential velocity $\langle v\rangle$ (Contour interval $3 \mathrm{~m} \mathrm{~s}^{-1}$ ); (c) vertical velocity $\langle w\rangle$ (Contour interval for positive $0.5 \mathrm{~m} \mathrm{~s}^{-1}$; negative contours -0.05 , $\left.-0.1,-0.15,-0.2 \mathrm{~m} \mathrm{~s}^{-1}\right)$; (d) sum of mean vorticity influx and vertical advection $V_{\mathrm{m} \zeta}+V_{\mathrm{mv}}$ (Contour interval: $2 \mathrm{~m} \mathrm{~s}^{-1} \mathrm{~h}^{-1}$ ); (e) radial (subgrid-scale) diffusive tendency $V_{\mathrm{d} r}$ (Contour interval $0.5 \mathrm{~m} \mathrm{~s}^{-1} \mathrm{~h}^{-1}$ ); (f) vertical (subgrid-scale) diffusive tendency $V_{\mathrm{d} z}($ Contour interval $\left.0.5 \mathrm{~m} \mathrm{~s}^{-1} \mathrm{~h}^{-1}\right)$; (g) mean tangential wind tendency $\partial\langle v\rangle / \partial t$ (Contour interval $2 \mathrm{~m} \mathrm{~s}^{-1} \mathrm{~h}^{-1}$ units); (h) sum of all tendency terms (Contour interval $\left.2 \mathrm{~m} \mathrm{~s}^{-1} \mathrm{~h}^{-1}\right)$.

As in the 3D3k simulation, the tendency terms in the AX3k simulation during the mature stage (Fig. 13d-g) exhibit a broadly similar structure to those found during the intensification period. The net tendency term is of course smaller on account of the fact that the vortex is near its maximum intensity.

\section{Eddy momentum fluxes}

In the previous section it was found that, while the mean vorticity influx and vertical advection comprise the leading terms of the tangential wind tendency, the resolved and parameterized (subgrid) eddy processes contribute nonnegligibly to the mean spin-up tendency around the eyewall and wind maximum throughout the troposphere. Here we examine the structure of these eddy fluxes comparing the 3-D and AX simulations during both spin-up and maturity.

\subsection{Flux form of the tangential momentum equation}

To establish the framework for comparing resolved and subgrid momentum fluxes, we first rewrite the tangential momentum equation of Sect. 5 in flux divergence form. For simplicity, we again adopt a Boussinesq approximation similar to that employed in Sect. $4^{12}$. With the same nomenclature as Sect. 5, the flux form of the azimuthally averaged tangential momentum tendency equation in cylindrical coordinates

\footnotetext{
${ }^{12}$ The simplified diagnosis can be justified on the grounds that more elaborate diagnoses using more cumbersome equations including triple correlations involving eddy momentum flux and perturbation density, as well as horizontal and vertical variation of azimuthal mean density yield virtually identical results to the simpler set (not shown). In other words, physical insight gleaned from the approximate tangential momentum equation is essentially the same as found using the less approximate anelastic or fully compressible formulations.
} 

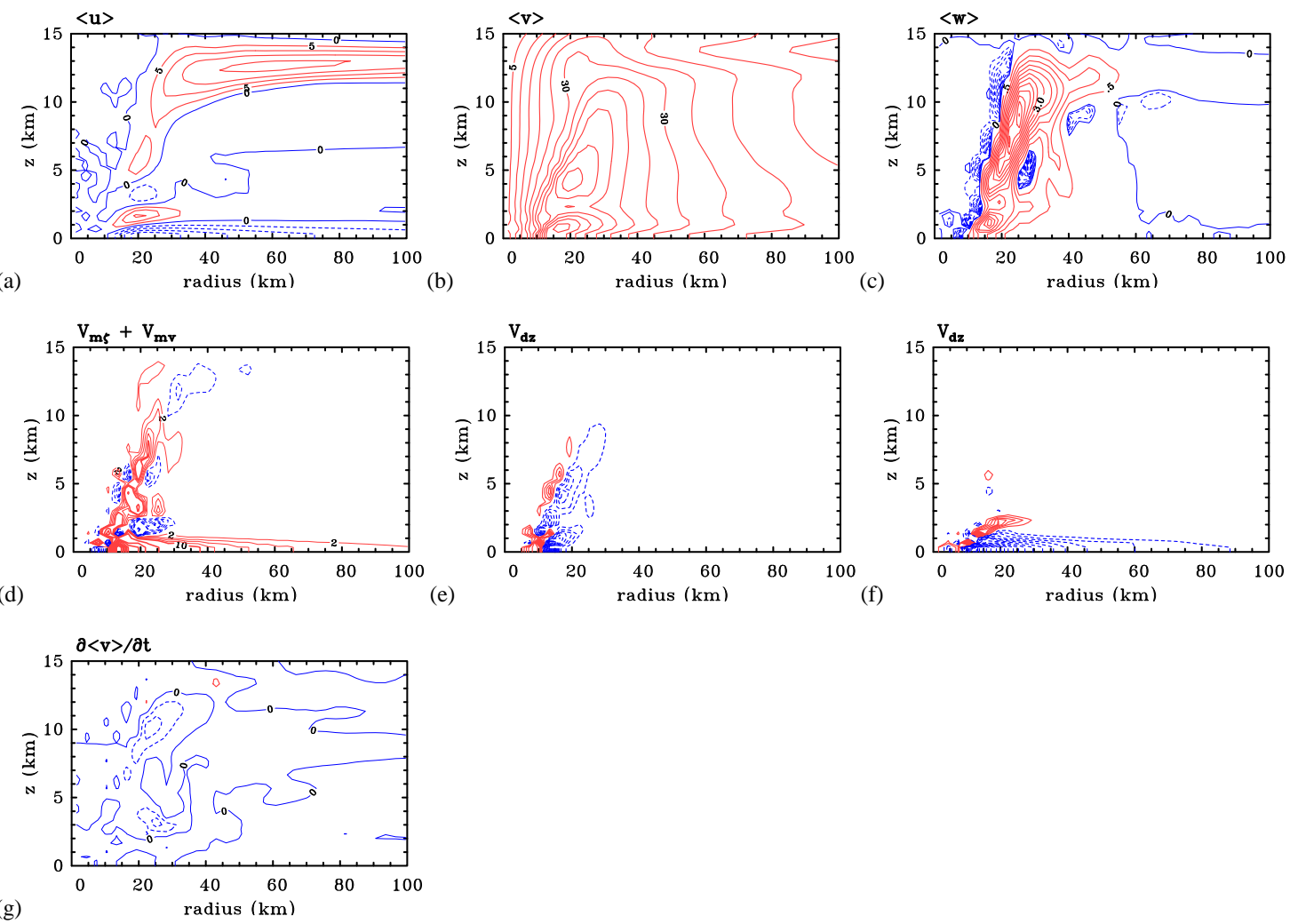

Fig. 13. Terms of the tangential wind tendency equation for the simulation AX3k, averaged during the mature stage $(176-200 \mathrm{~h})$. Red color denotes positive values; blue color denotes negative values. (a) Radial velocity $\langle u\rangle$ (Contour interval $5 \mathrm{~m} \mathrm{~s}^{-1}$ ); (b) tangential velocity $\langle v\rangle$ (Contour interval $5 \mathrm{~m} \mathrm{~s}^{-1}$ ); (c) vertical velocity $\langle w\rangle$ (Contour interval for positive $0.5 \mathrm{~m} \mathrm{~s}^{-1}$; negative contours $-0.05,-0.1,-0.15$, $-0.2 \mathrm{~m} \mathrm{~s}^{-1}$ ); (d) sum of mean vorticity influx and vertical advection $V_{\mathrm{m} \zeta}+V_{\mathrm{mv}}$ (Contour interval: thin, $2 \mathrm{~m} \mathrm{~s}^{-1} \mathrm{~h}^{-1}$; thick, $10 \mathrm{~m} \mathrm{~s}^{-1} \mathrm{~h}^{-1}$ ); (e) radial (subgrid-scale) diffusive tendency $V_{\mathrm{d} r}$ (Contour interval $2 \mathrm{~m} \mathrm{~s}^{-1} \mathrm{~h}^{-1}$ ); (f) vertical (subgrid-scale) diffusive tendency $V_{\mathrm{d} z}$ (Contour interval $\left.2 \mathrm{~m} \mathrm{~s}^{-1} \mathrm{~h}^{-1}\right)$; (g) mean tangential wind tendency $\partial\langle v\rangle / \partial t\left(\right.$ Contour interval $0.2 \mathrm{~m} \mathrm{~s}^{-1} \mathrm{~h}^{-1}$ ).

is

$$
\begin{aligned}
\frac{\partial\langle v\rangle}{\partial t}= & \frac{1}{r^{2}} \frac{\partial\left(-r^{2}\langle u\rangle\langle v\rangle\right)}{\partial r}+\frac{\partial(-\langle w\rangle\langle v\rangle)}{\partial z}-f\langle u\rangle \\
& +\frac{1}{r^{2}} \frac{\partial\left(-r^{2}\left\langle u^{\prime} v^{\prime}\right\rangle\right)}{\partial r}+\frac{\partial\left(-\left\langle v^{\prime} w^{\prime}\right\rangle\right)}{\partial z} \\
& +c_{\mathrm{p}}\left\langle\frac{\theta_{\rho}^{\prime}}{r} \frac{\partial \pi^{\prime}}{\partial \lambda}\right\rangle+\left\langle D_{v}\right\rangle .
\end{aligned}
$$

Again, $D_{v}$ is the subgrid-scale tendency expressed as a radius-height divergence of the subgrid momentum fluxes $\tau$ :

$\left\langle D_{v}\right\rangle=\frac{1}{r^{2}} \frac{\partial r^{2}\left\langle\tau_{r \lambda}\right\rangle}{\partial r}+\frac{\partial\left\langle\tau_{\lambda z}\right\rangle}{\partial z}$

where, for consistency with the Boussinesq-type of approximation here, the vertical variation of the basic state density has been neglected in the vertical derivative term in Eq. (17). The comparison of Eqs. (16) and (17) shows the direct analogy of resolved $-\left\langle u^{\prime} v^{\prime}\right\rangle$ and $-\left\langle v^{\prime} w^{\prime}\right\rangle$ with subgrid $\tau_{r \lambda}$ and $\tau_{\lambda z}$. In addition, in the mean radial and vertical momentum tendency equations (not written), the resolved $-\left\langle u^{\prime} w^{\prime}\right\rangle$ is the analog of subgrid $\tau_{r z}$. In the CM1 model (Sect. 2), the subgrid momentum fluxes are specified by local eddy diffusion relations (written here in cylindrical-polar coordinates),

$$
\begin{aligned}
& \left\langle\tau_{r \lambda}\right\rangle=\left\langle K_{\mathrm{m}, \mathrm{h}}\left(\frac{1}{r} \frac{\partial u}{\partial \lambda}+r \frac{\partial v / r}{\partial r}\right)\right\rangle \\
& \left\langle\tau_{\lambda z}\right\rangle=\left\langle K_{\mathrm{m}, \mathrm{v}}\left(\frac{1}{r} \frac{\partial w}{\partial \lambda}+\frac{\partial v}{\partial z}\right)\right\rangle
\end{aligned}
$$

with parameterization formulae for horizontal and vertical eddy diffusivities, $K_{\mathrm{m}, \mathrm{h}}$ and $K_{\mathrm{m}, \mathrm{v}}$. The analogous specification for $\tau_{r z}$

$\left\langle\tau_{r z}\right\rangle=\left\langle K_{\mathrm{m}, \mathrm{v}}\left(\frac{\partial u}{\partial z}+r \frac{\partial w / r}{\partial r}\right)\right\rangle$

is also shown in the figures below.

\subsection{Eddy flux analysis}

Figures 14-15 show the $(r, z)$ structure of resolved eddy fluxes, subgrid fluxes and their eddy diffusivities, and a local, 
moist Richardson number (defined below) for the two spinup periods in the 3-D simulation indicated in Fig. 1a. As noted in Sect. 3, a second intensification interval (151-155 h) is chosen for analysis in the 3-D model because the intensification period spans a comparatively longer time interval than in the AX model. The two periods for the 3-D model provide a representative sample of the radius-height eddy structure during the extended spin-up period. Figure 16 shows the corresponding fields for the AX simulation during the primary spin-up period indicated in Fig. 1a. By definition, there are no resolved eddy fluxes in the AX model. The selected spin-up times are centred on the times $t=67 \mathrm{~h}$ and $t=153 \mathrm{~h}$ for the 3-D simulation and $t=79 \mathrm{~h}$ for the AX simulation. Figures 17 and 18 show the analogous fields for the mature vortex phase in the 3-D and AX simulations, respectively. Each figure has the same multi-panel format as detailed in the captions. For the reader's convenience, we include in panels (a)-(c) of each figure the azimuthally averaged velocity component shown in the previous section, except for the second intensification phase.

In the analyses that follow a general conclusion is that the analogous resolved and subgrid fluxes are quite different from each other in pattern and usually in magnitude. It follows that the parameterization of eddy momentum flux in the AX model is inconsistent with the resolved fluxes determined by the 3-D model ${ }^{13}$.

In addition, the differences between the resolved and subgrid flux within each of these figures are generally greater than the subgrid $\tau$ differences between the 3-D and AX models and greater than the time period differences. Readers who prefer to skip the detailed description of the results leading to these conclusions may wish to jump to Sect. 6.5 below.

The quantities plotted in Figs. 14-18 are time averages over a specific interval. In the 3-D model, an averaging period of $4 \mathrm{~h}$ is used for the two intensification periods in Figs. 14 and 15, whereas the averaging period for mature phase is $12 \mathrm{~h}$ in Fig. 17. In the AX model, an averaging period of $6 \mathrm{~h}$ is used for the primary intensification period in Fig. 16, whereas the averaging period for the mature phase is $24 \mathrm{~h}$ in Fig. 18. A longer averaging period was chosen for the AX model because of the noisier fields compared to the 3$\mathrm{D}$ model. The averages are constructed from data output at 2 min intervals (except Fig. 17 that uses a 10 min interval) to provide an adequate resolution of the time variability of the different fields during the averaging period (i.e., the snapshot decorrelation time is much shorter than the averaging period). Because the standard deviation of each respective time series is found to be comparable or less than their time-mean

\footnotetext{
${ }^{13}$ This remark does not apply to the boundary layer where subgrid $\tau_{r z}$ and $\tau_{\lambda z}$ are dominant in both 3-D and AX models, as expected. Because the boundary layer story is addressed in Sects. 5 and 7, we focus in this section above the frictional boundary layer $(z \geq 1 \mathrm{~km})$.
}

counterpart $^{14}$, we conclude that these time-averaged quantities are adequate to characterize the qualitative structure of the plotted fields, although residual intrinsic (sampling) variability is evident as smaller-scale noise in the spatial patterns. This variability could be eliminated only by simulation ensembles with perturbed initial conditions, which is beyond the scope of this study. We show an extended time series of two momentum flux quantities and their associated tangential momentum tendencies in Fig. 19 to illustrate the time evolution of the eddy - mean interaction.

\subsection{Radial eddy momentum and vorticity flux}

During spin-up in the 3-D simulation, the resolved-eddy radial momentum flux, $-\left\langle u^{\prime} v^{\prime}\right\rangle$ (Figs. 14d and 15d), exhibits a coherent region of positive values around the RMW within and just above the boundary layer and extending upwards and outwards in the mean updraught to the middle troposphere. In the absence of an asymmetric secondary circulation, this inward-directed flux of cyclonic eddy momentum would contribute to a sharpening of the near eyewall horizontal shear and an increase of $\langle v\rangle$ inside the $\mathrm{RMW}^{15}$.

The evolutionary behaviour of the resolved eddy contribution is illustrated in Fig. 19 by a radius-time Hovmöller diagram of the eddy momentum flux, $-\left\langle u^{\prime} v^{\prime}\right\rangle$ and the vertical eddy tangential momentum flux, $-\left\langle w^{\prime} v^{\prime}\right\rangle$. Shown also are the eddy vorticity flux, $V_{\mathrm{e} \zeta}$ and the vertical advection of eddy tangential momentum, $V_{\mathrm{ev}}$, which appear in the material form of the mean tangential momentum Eq. (12). Since the maximum tangential wind during spin-up occurs near the top of the boundary layer, we have chosen to average all of these quantities in a layer near this altitude, between 1 and $2 \mathrm{~km}$ altitude.

Outside the RMW, $V_{\mathrm{e} \zeta}$ is generally negative throughout the simulation, implying a deceleration of $\langle v\rangle$ there. During the first intensification interval $(65-69 \mathrm{~h})$ and extending to approximately $t=140 \mathrm{~h},-\left\langle u^{\prime} v^{\prime}\right\rangle$ is mainly positive in a region straddling the RMW, and $V_{\mathrm{e} \zeta}$ is negative around the RMW and weakly positive $\left(\sim 0.3 \mathrm{~m} \mathrm{~s}^{-1} \mathrm{~h}^{-1}\right)$ further inside the RMW. These patterns broadly resemble a diffusive-like process in which the maximum tangential wind tends to be reduced while there is a spin-up tendency inside this radius. Between approximately $t=140 \mathrm{~h}$

\footnotetext{
${ }^{14}$ An exception occurs with $-\left\langle u^{\prime} v^{\prime}\right\rangle$, which has a standard deviation above the boundary layer that is consistently larger than the mean of the time series. Its spatial noise is also noticeably larger in Figs. 14d, 15d, and 17d than in other fields. However, individual snapshots recurrently show its dominant pattern near the RMW consisting of a positive outward-tilted feature below approximately $5 \mathrm{~km}$ and a negative outward-tilted feature above $6 \mathrm{~km}$ altitude.

${ }^{15}$ Since there is a locally strong secondary circulation component associated with vortical convection, the mean radial eddy vorticity flux is not simply equal to the mean radial divergence of the horizontal eddy momentum flux and an interpretation of the horizontal eddy dynamics is more challenging.
} 

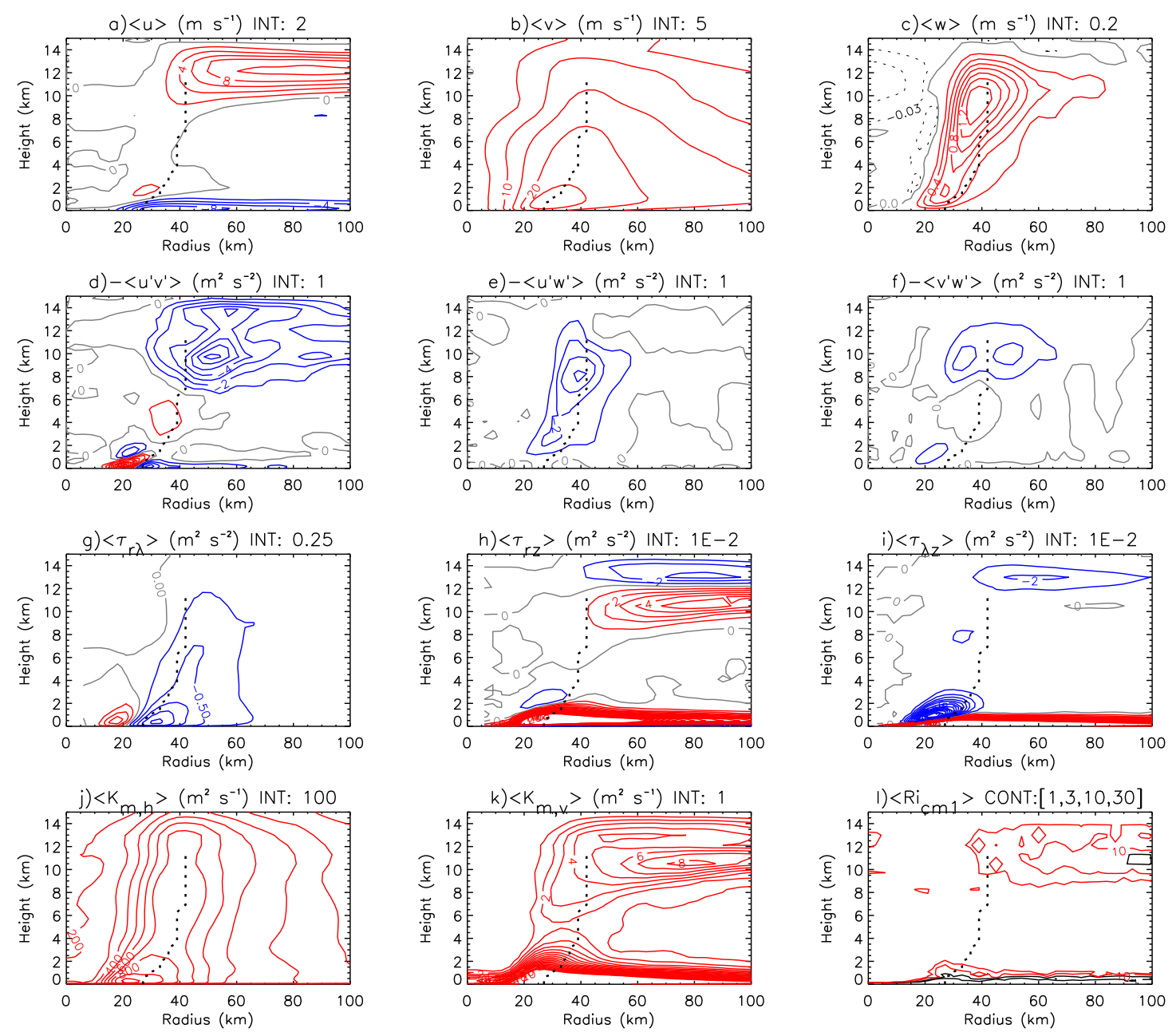

Fig. 14. Radius-height contour plots of resolved and subgrid-scale eddy momentum fluxes and related quantities from the 3D3k simulation using data at a 2 min interval and averaged over the first intensification interval $(65-69 \mathrm{~h})$, which is the interval of maximum intensification rate. Except where otherwise noted, there are 20 positive red contours and 20 negative blue contours. Panel (a) $\langle u\rangle$ with contour interval $2 \mathrm{~m} \mathrm{~s}^{-1}$; (b) $\langle v\rangle$ with contour interval $5 \mathrm{~m} \mathrm{~s}^{-1}$; (c) $\langle w\rangle$ with contour interval $0.2 \mathrm{~m} \mathrm{~s}^{-1}$; (d) resolved horizontal momentum flux $\left\langle-u^{\prime} v^{\prime}\right\rangle$ with contour interval $1 \mathrm{~m}^{2} \mathrm{~s}^{-2}$; (e) resolved vertical eddy flux of radial momentum $\left\langle-u^{\prime} w^{\prime}\right\rangle$ with contour interval $1 \mathrm{~m}^{2} \mathrm{~s}^{-2}$; (f) resolved vertical eddy flux of tangential momentum $\left\langle-v^{\prime} w^{\prime}\right\rangle$ with contour interval $1 \mathrm{~m}^{2} \mathrm{~s}^{-2}$; (g) subgrid momentum flux corresponding to panel (d): $\left\langle\tau_{r}\right\rangle$ with contour interval $0.25 \mathrm{~m}^{2} \mathrm{~s}^{-2}$; (h) subgrid momentum flux corresponding to (e) $\left\langle\tau_{r z}\right\rangle$ with contour interval $1 \times 10^{-2} \mathrm{~m}^{2} \mathrm{~s}{ }^{-2}$; (i) subgrid momentum flux corresponding to (f) $\left\langle\tau_{\lambda z}\right\rangle$ with contour interval $1 \times 10^{-2} \mathrm{~m}^{2} \mathrm{~s}^{-2}$; (j) horizontal diffusivity $\left\langle K_{\mathrm{m}, \mathrm{h}}\right\rangle$ with contour interval $100 \mathrm{~m}^{2} \mathrm{~s}^{-1} ;(\mathbf{k})$ vertical diffusivity $\left\langle K_{\mathrm{m}, \mathrm{v}}\right\rangle$ with contour interval $1 \mathrm{~m}^{2} \mathrm{~s}^{-1}$; (l) time and azimuthal average of the gradient Richardson number used in the CM1 model $\left\langle R i_{\mathrm{cm} 1}\right\rangle$ with black contours 1 and 3 and red contours 10 and 30 . The radius of maximum $\langle v\rangle$ at each height is shown with the black dashed curve (up to $11 \mathrm{~km}$ ).

and $t=165 \mathrm{~h}$ (including the second intensification interval highlighted above), $-\left\langle u^{\prime} v^{\prime}\right\rangle$ remains mostly positive in a region straddling the RMW, and $V_{\mathrm{e} \zeta}$ is recurrently positive and stronger in magnitude $\left(\sim 1 \mathrm{~m} \mathrm{~s}^{-1} \mathrm{~h}^{-1}\right)$ inside the RMW and negative outside the RMW. Unlike the earlier period, however, $V_{\mathrm{e} \zeta}$ is close to zero near the RMW. The fact the eddy vorticity flux is recurrently positive on the inside of the RMW and negative on the outside, implies that the eddies are not acting strictly diffusively and contribute to decreasing the RMW from approximately $30 \mathrm{~km}$ to $25 \mathrm{~km}$.
Figures 14 and 15 show that the subgrid radial momentum flux in the 3-D simulation is predominantly negative and much weaker in magnitude than the resolved-eddy flux near the RMW and within the mean updraught. Thus the resolved flux in the lower troposphere acts in a direction opposite to the local velocity gradient presumed by the subgrid scale model, i.e., it is counter-gradient. In the AX simulation, the subgrid flux (Fig. 16d) becomes comparable with that found in the 3-D simulation during the second intensification phase (Fig. 15g), but its radial dipole pattern in the 

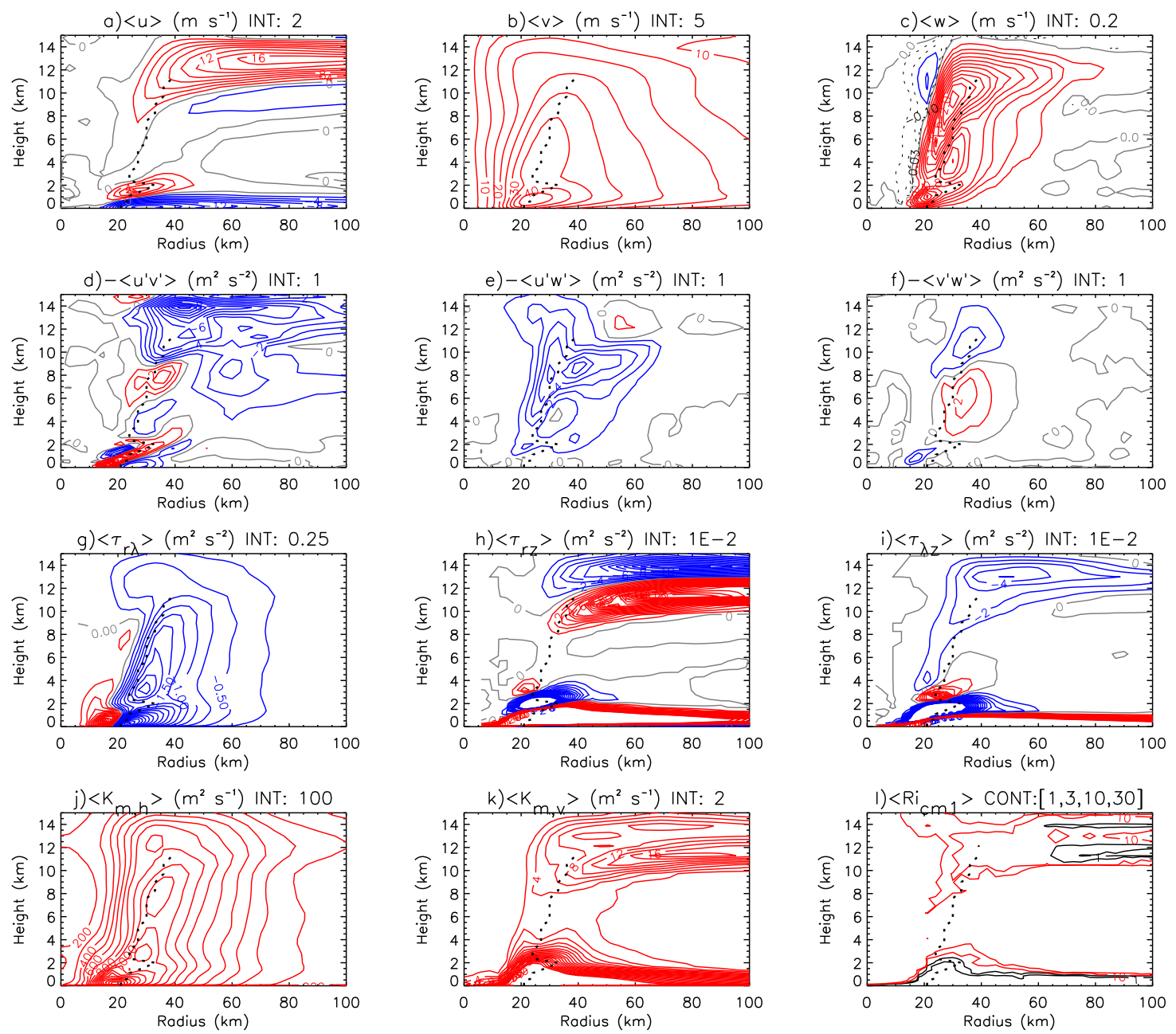

Fig. 15. Radius-height contour plots of resolved and subgrid-scale eddy momentum fluxes and related quantities from the 3D3k simulation using data at a 2 min interval and averaged over the second intensification interval (144-148 h). Contour intervals are the same as in Fig. 14. Except where otherwise noted, there are 20 positive red contours and 20 negative blue contours. (a) $\langle u\rangle$ with contour interval $2 \mathrm{~m} \mathrm{~s}^{-1}$; (b) $\langle v\rangle$ with contour interval $5 \mathrm{~m} \mathrm{~s}^{-1}$; (c) $\langle w\rangle$ with contour interval $0.2 \mathrm{~m} \mathrm{~s}^{-1}$; (d) resolved horizontal momentum flux $\left\langle-u^{\prime} v^{\prime}\right\rangle$ with contour interval $1 \mathrm{~m}^{2} \mathrm{~s}^{-2}$; (e) resolved vertical eddy flux of radial momentum $\left\langle-u^{\prime} w^{\prime}\right\rangle$ with contour interval $1 \mathrm{~m}^{2} \mathrm{~s}^{-2}$; (f) resolved vertical eddy flux of tangential momentum $\left\langle-v^{\prime} w^{\prime}\right\rangle$ with contour interval $1 \mathrm{~m}^{2} \mathrm{~s}^{-2}$; (g) subgrid momentum flux corresponding to (d) $\left\langle\tau_{r} \lambda\right\rangle$ with contour interval $0.25 \mathrm{~m}^{2} \mathrm{~s}^{-2}$; (h) subgrid momentum flux corresponding to (e) $\left\langle\tau_{r z}\right\rangle$ with contour interval $1 \times 10^{-2} \mathrm{~m}^{2} \mathrm{~s}^{-2}$; (i) subgrid momentum flux corresponding to (f) $\left\langle\tau_{\lambda z}\right\rangle$ with contour interval $1 \times 10^{-2} \mathrm{~m}^{2} \mathrm{~s}^{-2}$; (j) horizontal diffusivity $\left\langle K_{\mathrm{m}, \mathrm{h}}\right\rangle$ with contour interval $100 \mathrm{~m}^{2} \mathrm{~s}^{-1} ;(\mathbf{k})$ vertical diffusivity $\left\langle K_{\mathrm{m}, \mathrm{v}}\right\rangle$ with contour interval $2 \mathrm{~m}^{2} \mathrm{~s}^{-1}$; (l) time and azimuthal average of the gradient Richardson number used in the CM1 model $\left\langle R i_{\mathrm{cm} 1}\right\rangle$ with black contours 1 and 3 and red contours 10 and 30 . The radius of maximum $\langle v\rangle$ at each height is shown with the black dashed curve (up to $11 \mathrm{~km}$ ).

lower troposphere updraught region is essentially the reverse of the 3-D resolved-eddy pattern, and bear little resemblance to one another (Fig. 15d). This difference indicates an intrinsic limitation of the AX model.

The subgrid radial momentum fluxes in both simulations do not show any indication of the upper-troposphere broadening tendency of the resolved eddies. In the upper troposphere, the resolved-eddy flux is broadly negative, which implies a tendency to broaden the radial profile of $\langle v\rangle$ in the upper-tropospheric outflow region, and we see that it is broader in the 3-D simulation. This deficiency may have consequences for understanding the dynamics of the outflow layer.

In the mature phase (Figs. 17-18), all of the fluxes are larger than during spin-up. The resolved-eddy flux (Fig. 17d) is noisier than during spin-up (some of this noise is sampling variability, see above; and some is identified with the inertial recoil effect extending above the boundary layer as discussed 

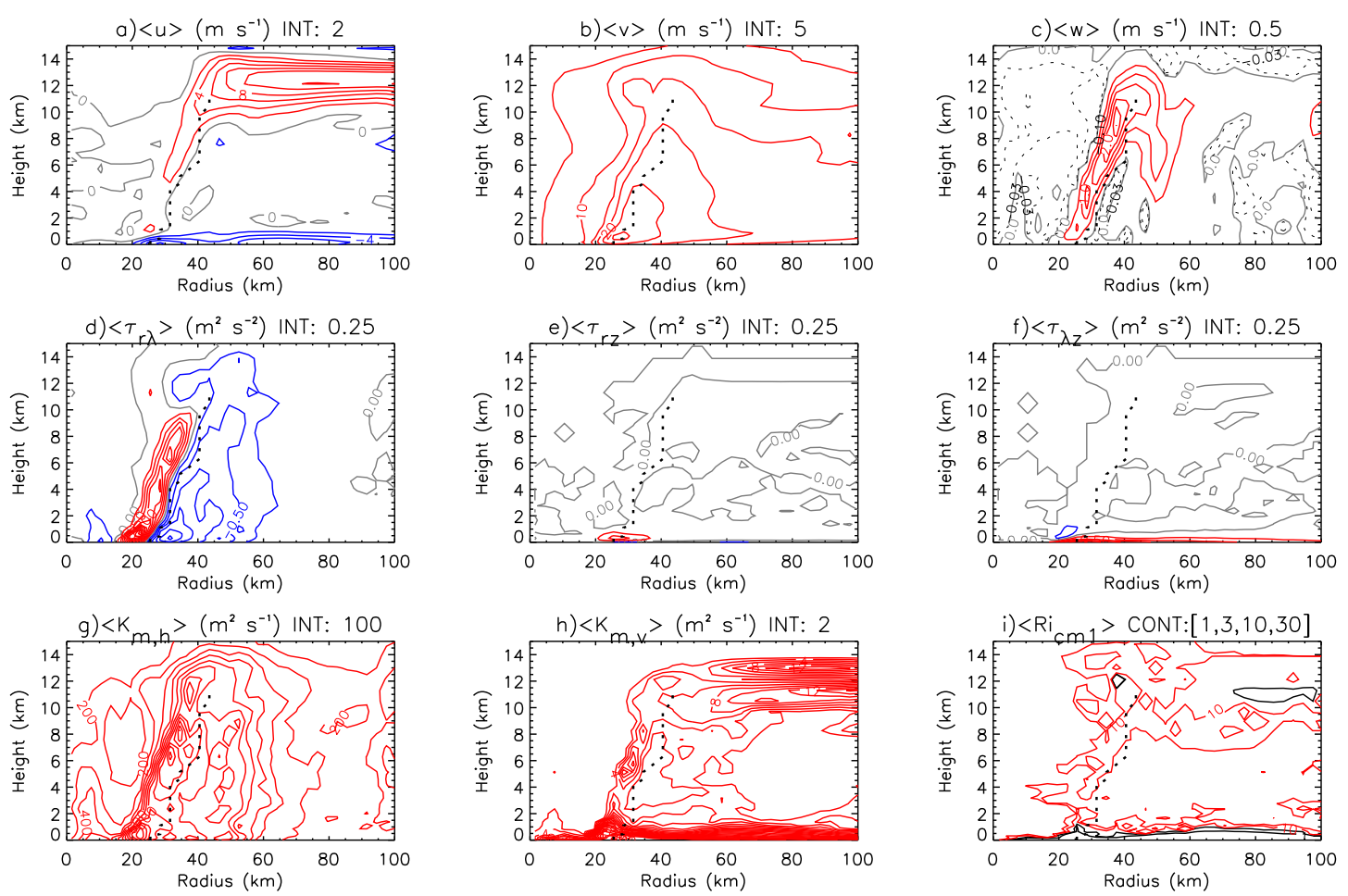

Fig. 16. Radius-height contour plots of resolved and subgrid eddy momentum fluxes and related quantities from the AX3k simulation using data at a 2 min interval and averaged over the interval of maximum intensification rate (77-83 h). Except where otherwise noted, there is a maximum of 20 positive red contours and 20 negative blue contours. (a) $\langle u\rangle$ with contour interval $2 \mathrm{~m} \mathrm{~s}^{-1}$; (b) $\langle v\rangle$ with contour interval $5 \mathrm{~m} \mathrm{~s}^{-1}$; (c) $\langle w\rangle$ with contour interval $0.5 \mathrm{~m} \mathrm{~s}^{-1}$; (d) subgrid momentum flux corresponding to Fig. 14d $\left.\left\langle\tau_{r}\right\rangle\right\rangle$ with contour interval $0.25 \mathrm{~m}^{2} \mathrm{~s}^{-2}$; (e) subgrid momentum flux corresponding to Fig. $14 \mathrm{e}\left\langle\tau_{r z}\right\rangle$ with contour interval $0.25 \mathrm{~m}^{2} \mathrm{~s}^{-2}$; (f) subgrid momentum flux corresponding to Fig. $14 \mathrm{f}\left\langle\tau_{\lambda z}\right\rangle$ with contour interval $0.25 \mathrm{~m}^{2} \mathrm{~s}^{-2}$; (g) horizontal diffusivity $\left\langle K_{\mathrm{m}, \mathrm{h}}\right\rangle$ with contour interval $100 \mathrm{~m}^{2} \mathrm{~s}^{-1}$; (h) vertical diffusivity $\left\langle K_{\mathrm{m}, \mathrm{v}}\right\rangle$ with contour interval $2 \mathrm{~m}^{2} \mathrm{~s}^{-1}$; (i) time and azimuthal average of the gradient Richardson number used in the CM1 model $\left\langle R i_{\mathrm{cm} 1}\right\rangle$ with black contours 1 and 3 and red contours 10 and 30 . The radius of maximum $\langle v\rangle$ at each height is shown with the black dashed curve (up to $11 \mathrm{~km}$ )

in Sect. 5.2), but it is broadly negative near the RMW and in the upper-tropospheric outflow. The subgrid flux in the 3-D simulation (Fig. 17g) is uniformly negative near the RMW and is significant in magnitude. In contrast to this simulation, the subgrid counterpart in the AX simulation (Fig. 18d) has essentially the same eddy-diffusive dipole pattern as during spin-up. Thus, as the system matures the resolved-eddy and subgrid scale fluxes have differences similar to those during spin-up, although they are perhaps less striking.

\subsection{Vertical momentum fluxes and eddy vertical advection}

The patterns of the 3-D resolved-eddy vertical fluxes (i.e., $-\left\langle u^{\prime} w^{\prime}\right\rangle$ and $-\left\langle v^{\prime} w^{\prime}\right\rangle$ in Figs. 14e, f, 15e, f and 17e, f) are tall, negative, outward-sloping columns concentrated around the RMW and mean updraught, both during spin-up and maturity. This location is where the vortical convective plumes are most active, and presumably they are the agents of these flux columns. The tendency of these flux columns is to ex- tend the region of strong tangential wind higher in the troposphere. As indicated by the vertical profile of $\langle v\rangle$, the mean tangential wind is more uniformly spread through the troposphere in the 3-D simulation, and the vertical shear of this wind is more concentrated aloft (Figs. $14 \mathrm{~b}$ and $17 \mathrm{~b}$ vs. 16b, $15 \mathrm{~b}$ and $18 \mathrm{~b}$ ); this difference is larger during spin-up than maturity.

In the layer average between 1 and $2 \mathrm{~km}$ (Fig. 19d), $-\left\langle w^{\prime} v^{\prime}\right\rangle$ is negative just inside the RMW and $V_{\mathrm{ev}}$ implies a positive tendency in this layer for $\langle v\rangle$ spanning the RMW especially just inside it, which is an acceleration tendency (cf. Fig. 13g). Both features persist throughout the spin-up period and maturity. The positive spin-up tendency associated with $V_{\mathrm{e} \zeta}$ near and inside the RMW increases from the approximate range 1 to $3 \mathrm{~m} \mathrm{~s}^{-1} \mathrm{~h}^{-1}$ early in the intensification period (50 to $70 \mathrm{~h}$ ) (cf. Fig. 13g) to the approximate range 3$10 \mathrm{~m} \mathrm{~s}^{-1} \mathrm{~h}^{-1}$ later in the intensification period (145 to $165 \mathrm{~h}$ ). Thus, comparatively speaking, the spin-up tendency from eddy vertical advection is roughly three times larger than that for the radial eddy vorticity flux (cf. Fig. 19b). 

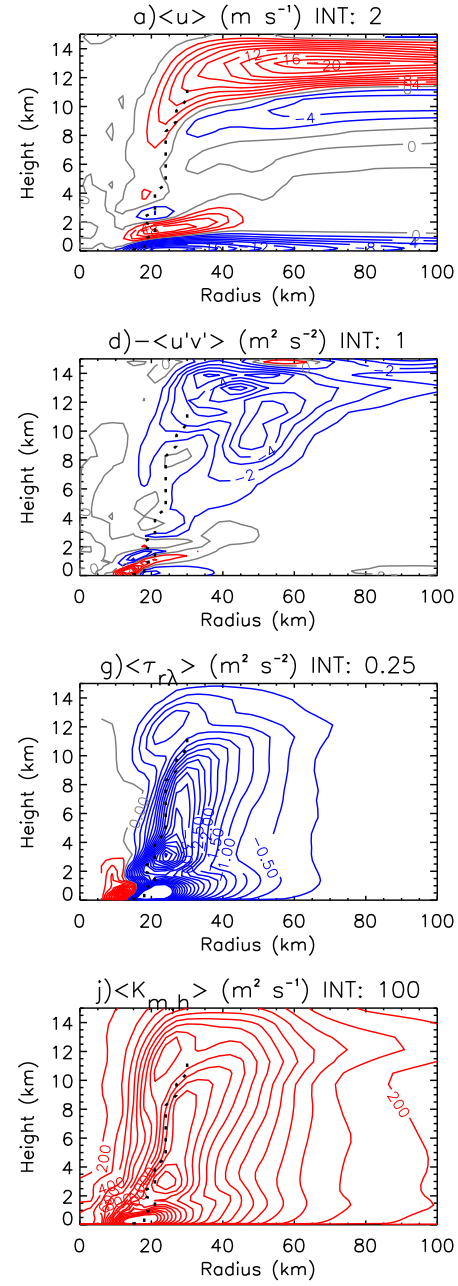
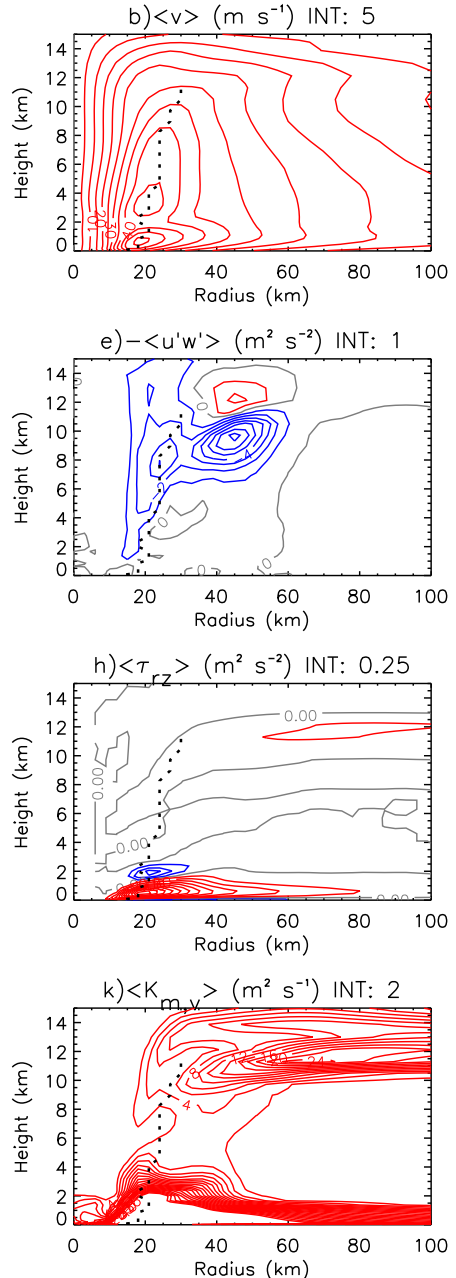
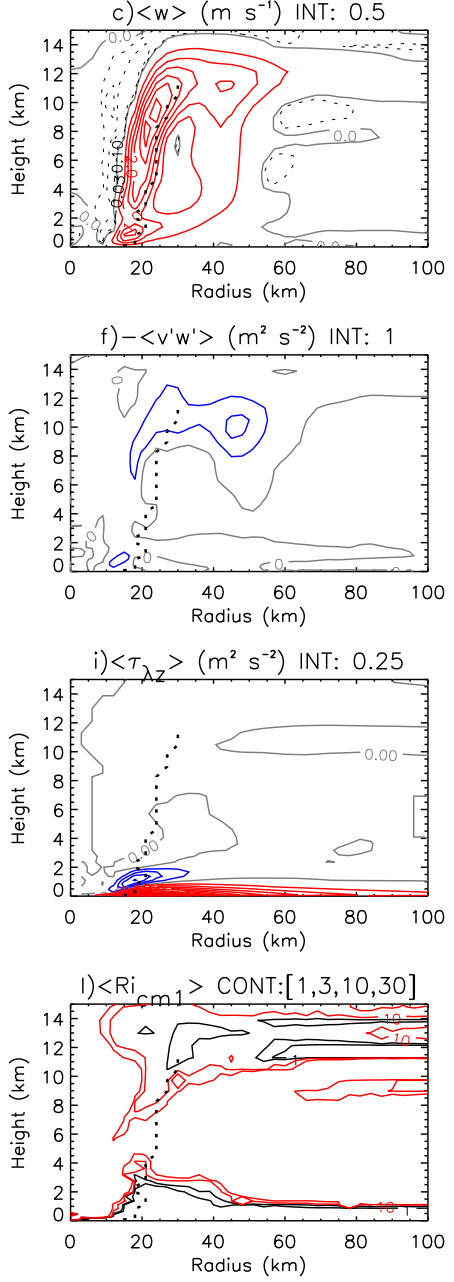

Fig. 17. Radius-height contour plots of resolved and subgrid eddy momentum fluxes and related quantities from the 3D3k simulation using data at a $10 \mathrm{~min}$ interval and averaged over a mature period (214-226 h). Except where otherwise noted, there are 20 positive red contours and 20 negative blue contours. (a) $\langle u\rangle$ with contour interval $2 \mathrm{~m} \mathrm{~s}^{-1}$; (b) $\langle v\rangle$ with contour interval $5 \mathrm{~m} \mathrm{~s}^{-1}$; (c) $\langle w\rangle$ with contour interval $0.5 \mathrm{~m} \mathrm{~s}^{-1} ;$ (d) resolved horizontal momentum flux $\left\langle-u^{\prime} v^{\prime}\right\rangle$ with contour interval $1 \mathrm{~m}^{2} \mathrm{~s}^{-2}$; (e) resolved vertical eddy flux of radial momentum $\left\langle-u^{\prime} w^{\prime}\right\rangle$ with contour interval $1 \mathrm{~m}^{2} \mathrm{~s}^{-2}$; (f) resolved vertical eddy flux of tangential momentum $\left\langle-v^{\prime} w^{\prime}\right\rangle$ with contour interval $1 \mathrm{~m}{ }^{2} \mathrm{~s}{ }^{-2}$; (g) subgrid momentum flux corresponding to (d) $\left\langle\tau_{r \lambda}\right\rangle$ with contour interval $0.25 \mathrm{~m}^{2} \mathrm{~s}^{-2}$; (h) subgrid momentum flux corresponding to (e) $\left\langle\tau_{r z}\right\rangle$ with contour interval $10 \times 10^{-3} \mathrm{~m}^{2} \mathrm{~s}^{-2}$; (i) subgrid momentum flux corresponding to $(\mathbf{f})\left\langle\tau_{\lambda z}\right\rangle$ with contour interval $10 \times 10^{-3} \mathrm{~m}^{2} \mathrm{~s}^{-2} ;(\mathbf{j})$ horizontal diffusivity $\left\langle K_{\mathrm{m}, \mathrm{h}}\right\rangle$ with contour interval $200 \mathrm{~m}^{2} \mathrm{~s}^{-1} ;(\mathbf{k})$ vertical diffusivity $\left\langle K_{\mathrm{m}, \mathrm{v}}\right\rangle$ with contour interval $2 \mathrm{~m}^{2} \mathrm{~s}^{-1} ;$ (l) time and azimuthal average of the gradient Richardson number used in the CM1 model $\left\langle R i_{\mathrm{cm} 1}\right\rangle$ with black contours 1 and 3 and red contours 10 and 30. The radius of maximum $\langle v\rangle$ at each height is shown with the black dashed curve (up to $11 \mathrm{~km}$ ).

Recall from Sect. 3, it is during the period around $66 \mathrm{~h}$ when the rate of spin-up of the mean tangential wind in the 3-D model exceeds the maximum spin-up rate of the AX model (cf. Fig. 1a). We showed also in Fig. 5c that at this time there were isolated regions of strong convective heating rate organized in a ring-like developing eyewall. These regions of strong heating will correspond with a strong positive signature in the $V_{\mathrm{ev}}$ field and would provide a plausible explanation for the enhanced spin-up rate in the 3-D model despite the relative weakness of the azimuthal mean heating rate in this model.
Recall that the tendency in $\langle u\rangle$ by the resolved flux, $-\left\langle u^{\prime} w^{\prime}\right\rangle$, is obtained via a vertical partial derivative of this flux. An examination of Figs. 14 and 15 reveals that the indicated tendency in $\langle u\rangle$ is to strengthen both lowertropospheric inflow and upper-tropospheric outflow, i.e., to accelerate the mean overturning circulation. Thus the resolved-eddy flux, $-\left\langle u^{\prime} w^{\prime}\right\rangle$, does not act like eddy diffusion, but represents a vertical non-local flux by the tropospherefilling vortical plumes.

During both the spin-up and mature phases the subgrid vertical fluxes in the 3-D and AX simulations (Figs. 14h, 

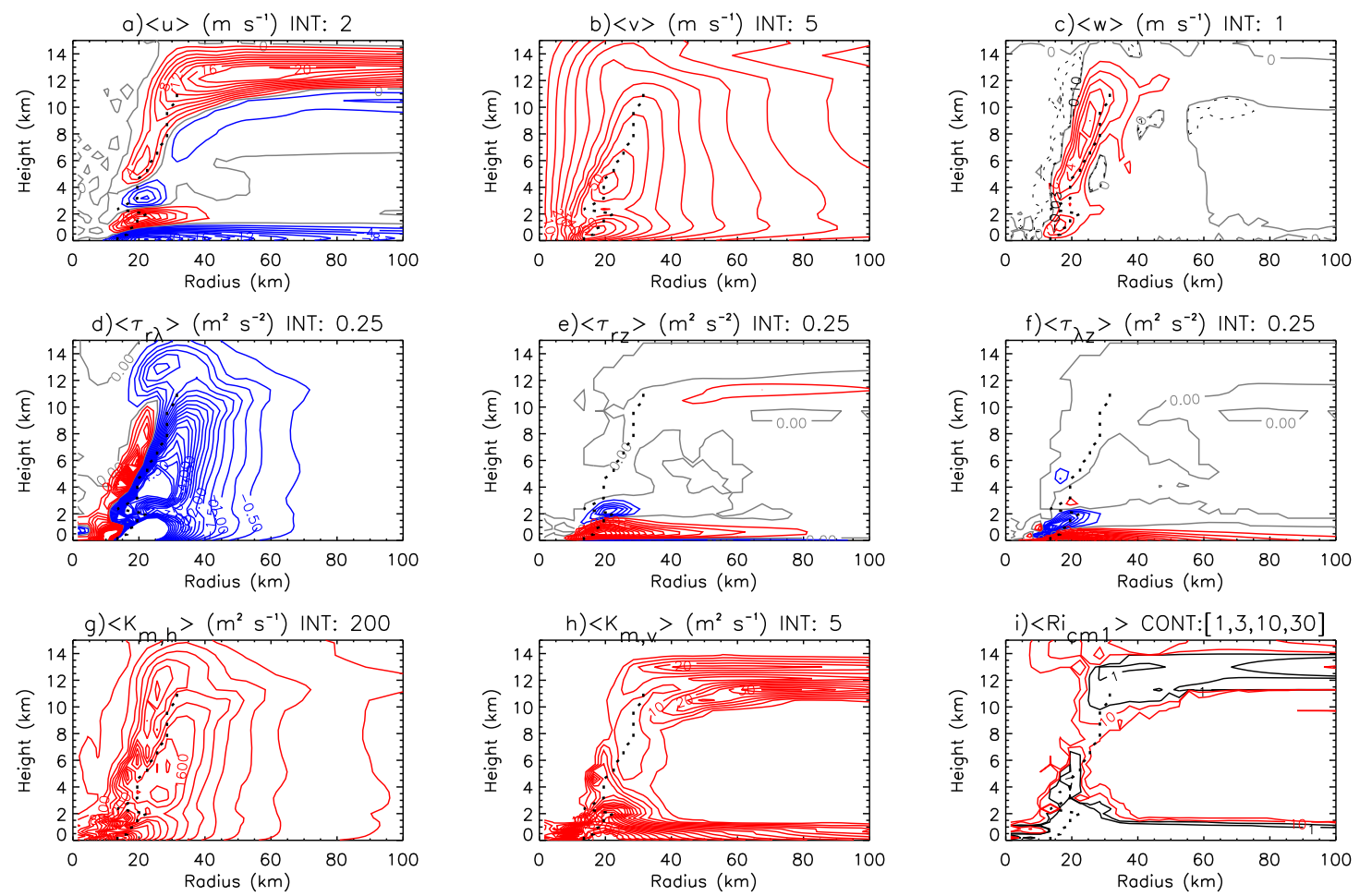

Fig. 18. Radius-height contour plots of resolved and subgrid eddy momentum fluxes and related quantities from the AX3k simulation using data at a 2 min interval and averaged over a mature period (176-200 h). Except where otherwise noted, there are 20 positive red contours and 20 negative blue contours. (a) $\langle u\rangle$ with contour interval $2 \mathrm{~m} \mathrm{~s}^{-1}$; (b) $\langle v\rangle$ with contour interval $5 \mathrm{~m} \mathrm{~s}^{-1}$; (c) $\langle w\rangle$ with contour interval $1 \mathrm{~m} \mathrm{~s}^{-1}$; (d) subgrid momentum flux corresponding to Fig. $17 \mathrm{~d}\left\langle\tau_{r \lambda}\right\rangle$ with contour interval $0.25 \mathrm{~m}^{2} \mathrm{~s}^{-2}$; (e) subgrid momentum flux corresponding to Fig. $17 \mathrm{e}\left\langle\tau_{r z}\right\rangle$ with contour interval $0.25 \mathrm{~m}^{2} \mathrm{~s}^{-2}$; (f) subgrid momentum flux corresponding to Fig. 17f $\left\langle\tau_{\lambda z}\right\rangle$ with contour interval $0.25 \mathrm{~m}^{2} \mathrm{~s}^{-2}$; (g) horizontal diffusivity $\left\langle K_{\mathrm{m}, \mathrm{h}}\right\rangle$ with contour interval $200 \mathrm{~m}^{2} \mathrm{~s}^{-1}$; (h) vertical diffusivity $\left\langle K_{\mathrm{m}, \mathrm{v}}\right\rangle$ with contour interval $5 \mathrm{~m}^{2} \mathrm{~s}^{-1}$; (i) time and azimuthal average of the gradient Richardson number used in the CM1 model $\left\langle R i_{\mathrm{cm} 1}\right\rangle$ with black contours 1 and 3 and red contours 10 and 30 . The radius of maximum $\langle v\rangle$ at each height is shown with the black dashed curve (up to $11 \mathrm{~km}$ ).

i; 16e, f; 17h, i; 18e, f) have the expected large extrema in the boundary layer, but they show nothing in the tropospheric RMW-updraught region where the resolved-eddy fluxes are active. There is some pattern similarity in the negative $-\left\langle v^{\prime} w^{\prime}\right\rangle$ and $\left\langle\tau_{\lambda z}\right\rangle$ in the upper-troposphere updraught region in the 3-D simulation, but the latter is much smaller, and in the AX simulation this feature is entirely absent. For the 3-D configuration, $\tau_{r z}$ has a weak vertical dipole pattern in the upper-tropospheric outflow region. Using the same argument as in the previous paragraph, this pattern implies a weak tendency in the vertical profile of $\langle u\rangle$ to decrease the outflow altitude. The associated $K_{\mathrm{m}, \mathrm{v}}$ have local maxima in the outflow region (Figs. 14k, 15k, 16h, 17k, 18h). However, in the 3-D simulation, there is no support for this effect in the resolved flux patterns in the upper-tropospheric outflow.

In summary, both radial and vertical resolved-eddy fluxes have qualitatively different patterns above the boundary layer in the 3-D simulation compared to the subgrid eddy-diffusive fluxes in either the 3-D or AX simulations, and the resolvededdy fluxes are generally larger in magnitude, especially the vertical fluxes. The largest resolved-eddy fluxes occur in the RMW-updraught region where vortical plumes are active. Their disparity with the subgrid patterns belies a simple interpretation as local momentum mixing.

\subsection{Discussion of eddy dynamics}

Having summarized the main features of the resolved- and subgrid-eddy fluxes in the 3-D and AX models, we examine now some additional physical characteristics of the horizontal and vertical eddies and illustrate their structure during a sample interval within the second intensification period.

First we recall that because the subgrid flux is based on the assumption of an "eddy-diffusion process" by definition, with $K_{\mathrm{m}, \mathrm{h}}$ a maximum in the RMW region (Figs. $14 \mathrm{j}, 15 \mathrm{j}$, and $16 \mathrm{~g}$ ), it acts to weaken and spread the peak in $\langle v\rangle$ as in a pure barotropic instability process (e.g., Schubert et al., 1999, their Fig. 4b). Although the foregoing results already indicate that the horizontal eddy dynamics are not acting strictly diffusively on the tangential wind, a natural question arises whether the resolved horizontal eddy vorticity 

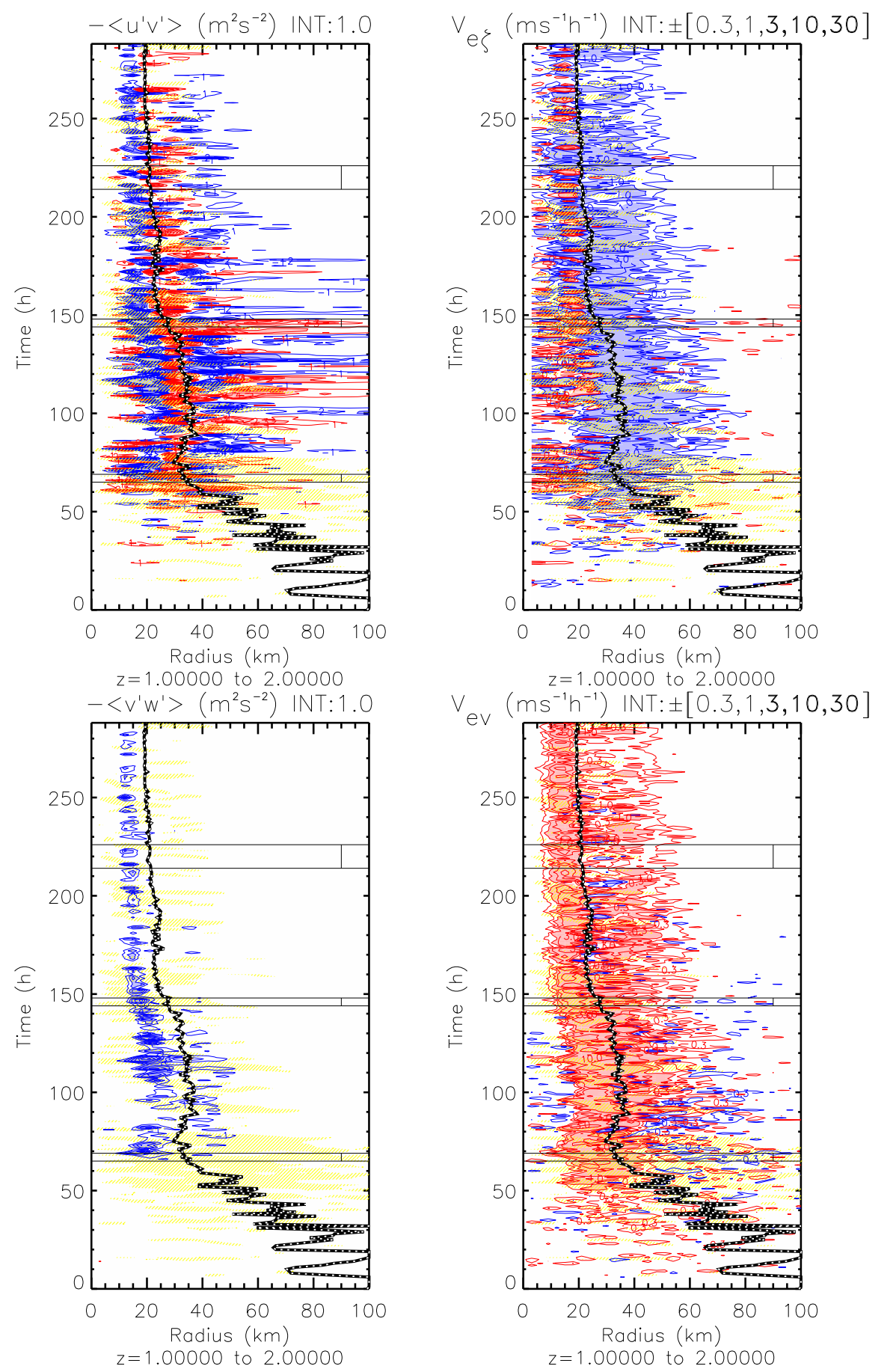

Fig. 19. Radius-time (Hovmöller) plots of layer-averaged (a) horizontal eddy momentum flux $\left(-\left\langle u^{\prime} v^{\prime}\right\rangle\right.$ ), (b) radial eddy vorticity flux ( $\left.V_{\mathrm{e} \zeta}\right)$, (c) vertical eddy tangential momentum flux $\left(-\left\langle w^{\prime} v^{\prime}\right\rangle\right)$ and (d) vertical advection of eddy tangential momentum ( $\left.V_{\text {ev }}\right)$, averaged between 1 and $2 \mathrm{~km}$ altitude from the $3 \mathrm{D} 3 \mathrm{k}$ simulation. (a) Contour interval $\pm 1.0 \mathrm{~m}^{2} \mathrm{~s}^{-2}$; (b) contours, $\pm 0.3,1,3,10$ and $30 \mathrm{~m} \mathrm{~s}^{-1} \mathrm{~h}^{-1}$; (c) contour interval $\pm 1.0 \mathrm{~m}^{2} \mathrm{~s}^{-2}$; (d) contours $\pm 0.3,1,3,10$ and $30 \mathrm{~m} \mathrm{~s}^{-1} \mathrm{~h}^{-1}$. Red contours denote positive values and blue contours denote negative values. Spotted black-curve denotes layer-averaged RMW. Yellow shaded area denotes regions where the layer-averaged tangential wind tendency is positive and exceeds $0.25 \mathrm{~m} \mathrm{~s}^{-1} \mathrm{~h}^{-1}$. Horizontal black lines denote the time intervals analysed in detail in this paper. 
flux in the 3D3k simulation supports the barotropic instability paradigm. Although Fig. 8b suggests that a ring-like potential vorticity structure is evident during the first intensification period, animations of the vorticity field on level surfaces show that the vorticity field is highly disorganized and not ring-like during this time (cf. also Fig. 2). The ringlike structure in Fig. 8b is rather an artifact of azimuthally averaging highly localized cyclonic vorticity anomalies just inside the RMW. Thus one of the necessary conditions for (linear) barotropic-baroclinic instability in a rapidly rotating baroclinic vortex is not satisfied during the first intensification period (Montgomery and Shapiro, 1995). At later times $(t>80 \mathrm{~h})$, a more ring-like vorticity structure does emerge (cf. Fig. 2), but a significant limitation of applying purely adiabatic shear instability theory is that the eyewall region is being persistently forced by convection on timescales that are typically short (tens of minutes) compared to barotropic instability e-folding times (hours to $10 \mathrm{~h}$, Schubert et al., 1999, Nguyen et al., 2011). Even if the necessary conditions for (linear) instability are satisfied, Schecter and Montgomery (2007) indicated that, when the effects of cloudiness are accounted for, the predicted e-folding times may be increased significantly (by a factor of three for a hurricane-like vortex example), depending on the extent of cloudiness. For these reasons, Nguyen et al. (2011) proposed the idea of a mixed barotropic-convective instability to explain a transition from a more symmetric to a more asymmetric phase during the intensification phase of their simulation of Hurricane Katrina (2005). However, the disparity in timescales between the convective instability and moist barotropic instability remains a significant issue.

The moist barotropic instability paradigm for ring-like potential vorticity distributions corresponding to a developed eyewall updraught does offer a plausible interpretation for the negative eddy vorticity flux forcing found near and inside the RMW during the early intensification period (Fig. 19b). However, the foregoing discussion is a reminder that one must be cautious of such a simple interpretation for a flow situation in which deep precipitating convection and frictional forcing are important processes in the eyewall vorticity balance. As an example, Fig. 19b shows that the associated radial eddy vorticity flux promotes the spin-up of the mean vortex just inside the RMW, but often leaves the maximum tangential wind approximately unchanged. Although this spin-up contribution inside the RMW is consistent with pure moist barotropic instability of an elevated annulus of relative vorticity, as noted above the near-zero eddy tendency at the RMW is not consistent with a diffusive weakening of the maximum tangential wind.

As noted in the foregoing section, as the vortex progressively strengthens, there is a period in which the eddy vorticity flux forcing becomes significantly positive inside the RMW ( $140 \mathrm{~h}<t<165 \mathrm{~h})$ and the RMW continues to contract inwards. An alternative explanation to the barotropic instability model is suggested upon examining animations of the simulated vorticity field in the lower troposphere of the vortex. During sub-periods of positive spin-up tendency, the animations suggest a sequence of local cyclonic vorticity generation, followed by a tendency of vortex axisymmetrization and trailing-spiral vorticity bands, which partially encircle the cyclonic mesoscale vorticity region near the RMW. These banded features appear plausibly consistent with convectively coupled sheared vortex Rossby waves that would act to strengthen the mean vortex at radii interior to their excitation radius (Montgomery and Kallenbach, 1997; Montgomery and Enagonio, 1998; Wang, 2002a, b; Chen et al., 2003; McWilliams et al., 2003). We pause now to elucidate further the physical nature of the horizontal and vertical eddies in the 3D3k simulation during the second period of intensification.

To identify the physical nature of the eddy spin-up contribution, the left column of Fig. 20 shows the vertical vorticity $\zeta$ and vertical velocity $w$ at the $z=1.5 \mathrm{~km}$ level for a sequence of times, spaced $6 \mathrm{~min}$ apart. The RMW during this analysis interval is highlighted by the spotted circle at $r=24 \mathrm{~km}$. The chosen time interval isolates a particularly positive period of radial eddy vorticity flux forcing near the RMW (i.e., $V_{\mathrm{e} \zeta}>0$ ) during the identified second intensification period ${ }^{16}$. During this period, several banded features of vorticity are seen to rotate cyclonically around the approximately circular vorticity ring associated with the eyewall. Of interest here are the two banded features that extend to the north (in the direction of $y$ ) from the eyewall. These two bands are associated with enhancements in vertical velocity and thus can be considered convectively coupled (or convectively generated).

The panels in the centre of Fig. 20 show perturbation radial velocity, perturbation vertical vorticity and the radial eddy vorticity flux $-u^{\prime} \zeta^{\prime}$ (plotted only for values exceeding $3 \mathrm{~m} \mathrm{~s}^{-1} \mathrm{~h}^{-1}$ magnitude). The principal vorticity asymmetry at the RMW is found in the northwest sector, the point of intersection of the more prominent of the two northern vorticity bands. This vorticity asymmetry rotates counterclockwise about the vortex centre and is associated with the strongest updraught found in the eyewall (darkest green shading for $w>3 \mathrm{~m} \mathrm{~s}^{-1}$ in the left column). Since the term associated with the spin-up of the mean tangential wind by the eddy vorticity flux is $-u^{\prime} \zeta^{\prime}$, a positive value of this quantity, after averaging around a circle of constant radius, would promote intensification. At the first time, positive values of $-u^{\prime} \zeta^{\prime}$ at $r=20 \mathrm{~km}$ are mostly attributable to the intersection of the

\footnotetext{
${ }^{16}$ Identification was made with a version of Fig. 19 refined to the second intensification period. While the feature illustrated here is not representative of the entire time period, as negative forcing inside the RMW occupies much of the time period, this episode is found to be typical of this and four other episodes (i.e. a total of five such positive episodes over an extended eight hour period) during which the azimuthally averaged tangential wind intensifies by about $5 \mathrm{~m} \mathrm{~s}^{-1}$ and the RMW contracts by about $5 \mathrm{~km}$ (from 30 to $25 \mathrm{~km}$, averaged over this layer).
} 
vorticity band from the north with the eyewall. As the vorticity enhancement rotates around the eyewall, the more interior portion of this feature is associated with positive values of $-u^{\prime} \zeta^{\prime}$ due to the eddy inflow there (black dotted line $u^{\prime}<-1 \mathrm{~m} \mathrm{~s}^{-1}$ ). The vorticity perturbation exceeds $10 \times 10^{-4}$ $\mathrm{s}^{-1}$ in magnitude. This vorticity enhancement is collocated with the vertical velocity enhancement and is favourably juxtaposed with asymmetric inflow, thus promoting localized spin-up. Although there is a modest region found in the eastern sector of the eyewall featuring negative values of $-u^{\prime} \zeta^{\prime}$, the positive values of the local eddy flux found in the west outweigh the negative values found in the east.

Downstream of the positive eddy vorticity feature in the eyewall, the vertical eddy tendency term (shown in the right column) indicates a spin-up tendency (pink) that is coincident with an arc-like feature of eddy vertical velocity (thin black solid contour is $w^{\prime}>1 \mathrm{~m} \mathrm{~s}^{-1}$ ) in the west sector, which rotates cyclonically to the southern sector where $w^{\prime}>0$ and $\partial v^{\prime} / \partial z<0$. In the eastern and northern sectors, there are areas of positive tendency also where $w^{\prime}<0$ and $\partial v^{\prime} / \partial z>0$. According to Fig. 19, the anticorrelation between $w^{\prime}$ and $\partial v^{\prime} / \partial z$ typifies the vertical eddy contribution to the tangential wind tendency in the eyewall region. Recalling the boundary layer spin-up mechanism discussed in the Introduction and illustrated in Sect. 5, the eddy vertical velocity is highly correlated with eddy tangential velocity, because of the convective transport of tangential momentum from low levels. In the vicinity of the eyewall, the flow above the boundary layer and the asymmetries thereof tilt radially outwards with height. The outward tilt of these features with height between 20 and $40 \mathrm{~km}$ radius implies that positive or negative asymmetries of tangential velocity will be anticorrelated with the vertical derivative of the tangential wind asymmetry, i.e., $-w^{\prime} \partial v^{\prime} / \partial z>0$.

Outside the eyewall, vorticity bands (whether positive or negative) frequently exhibit positive or negative tendency in $-u^{\prime} \zeta^{\prime}$. Isolated convection outside the eyewall leads to cellular enhancements of vorticity and for reasons offered in the prior subsection, these vorticity anomalies may be associated with a spin-up or spin-down signal in $-u^{\prime} \zeta^{\prime}$. The eddy radial velocity exhibits larger spatial scales of variability and the smaller-scale convective signals in vorticity appear to be generated within eddy outflow or eddy inflow, more-or-less evenly.

In summary, the principal cause of the net positive tendency in the radial eddy vorticity flux in the eyewall (just inside the RMW) is the horizontal flow signatures resulting from convective-vorticity asymmetries found near and inside the eyewall. An asymmetry in the inflow is found to support the convective asymmetry that, together with the induced vorticity asymmetry, yields a negative correlation between the eddy radial wind and the eddy vorticity and thus a positive acceleration of the mean tangential wind there. These results are suggestive that the alternative explanation of convectively generated vorticity and its subsequent tendency to axisymmetrize is the most plausible during periods of positive eddy-vorticity-flux forcing during the second intensification interval. The above analyses show also that the phenomena of vertical eddy acceleration of both the tangential and secondary circulations by convective vortices is quantitatively larger than that of the horizontal eddy acceleration. At the present time we lack a full thermo-mechanical explanation of the eddy dynamics.

Finally, we remark that an alternative analysis of the azimuthally averaged angular momentum balance (i.e., for $\langle M\rangle=r\langle v\rangle+\frac{1}{2} f r^{2}$ ) shows remarkable similarity in the patterns and relative magnitudes of the horizontal and vertical eddy fluxes to those shown in Figs. 10g, 14d, f, 15d, f, and 19 for tangential momentum.

\subsection{Richardson number}

The gradient-Richardson number (panels 1 for the 3-D simulation or i for the AX simulation), in conjunction with the time-averaged vertical eddy diffusivity (panels $\mathrm{k}$ or $\mathrm{h}$ for the two calculations, respectively), serves as an indicator for the possible occurrence of moist Kelvin-Helmholtz (stratifiedshear) instability and parameterized small-scale vertical mixing processes above the boundary layer. An investigation of both quantities allows also a first-assessment of a newly proposed theory of tropical cyclone intensification by Emanuel (2012). The "self-stratification" hypothesis posits that smallscale mixing processes associated with stratified-shear instabilities in the upper-level eyewall and outflow region control the intensifying vortex by limiting the Richardson number to a critical value near unity in the upper-tropospheric outflow layer. The idea is that the mixing sets the thermal stratification of the outflow region of the storm. The Richardson number plotted here is the azimuthal and time average of the local Richardson number $R i_{\mathrm{cm} 1}$ used in the CM1 model for determining where small-scale vertical mixing associated with shear-stratified turbulence occurs:

$$
R i_{\mathrm{cm} 1}=\frac{N_{\mathrm{m}}{ }^{2}}{\left(\frac{\partial u}{\partial z}\right)^{2}+\left(\frac{\partial v}{\partial z}\right)^{2}},
$$

where $N_{\mathrm{m}}{ }^{2}$ is the local moist static stability as defined exactly in the CM1 model. When this Richardson number is non-negative and falls below unity, the shear-stratified turbulence parameterization is activated and the vertical eddy diffusivity is nonzero. During the spin-up phases in the 3-D model, Figs. 141 and 151 show a widespread region in the upper-level eyewall and outflow region where the mean gradient Richardson number varies typically between 10 and 30 , well above the posited critical value of unity. Of course, given the variability of the solution illustrated by Fig. 1, we would expect some deviation above and below these average values during the spin-up period. However, for a given radius and height, the azimuth-time percentage of realizations in which the Richardson number drops below unity is found to be no more than $30 \%$. In other words, the instances when 

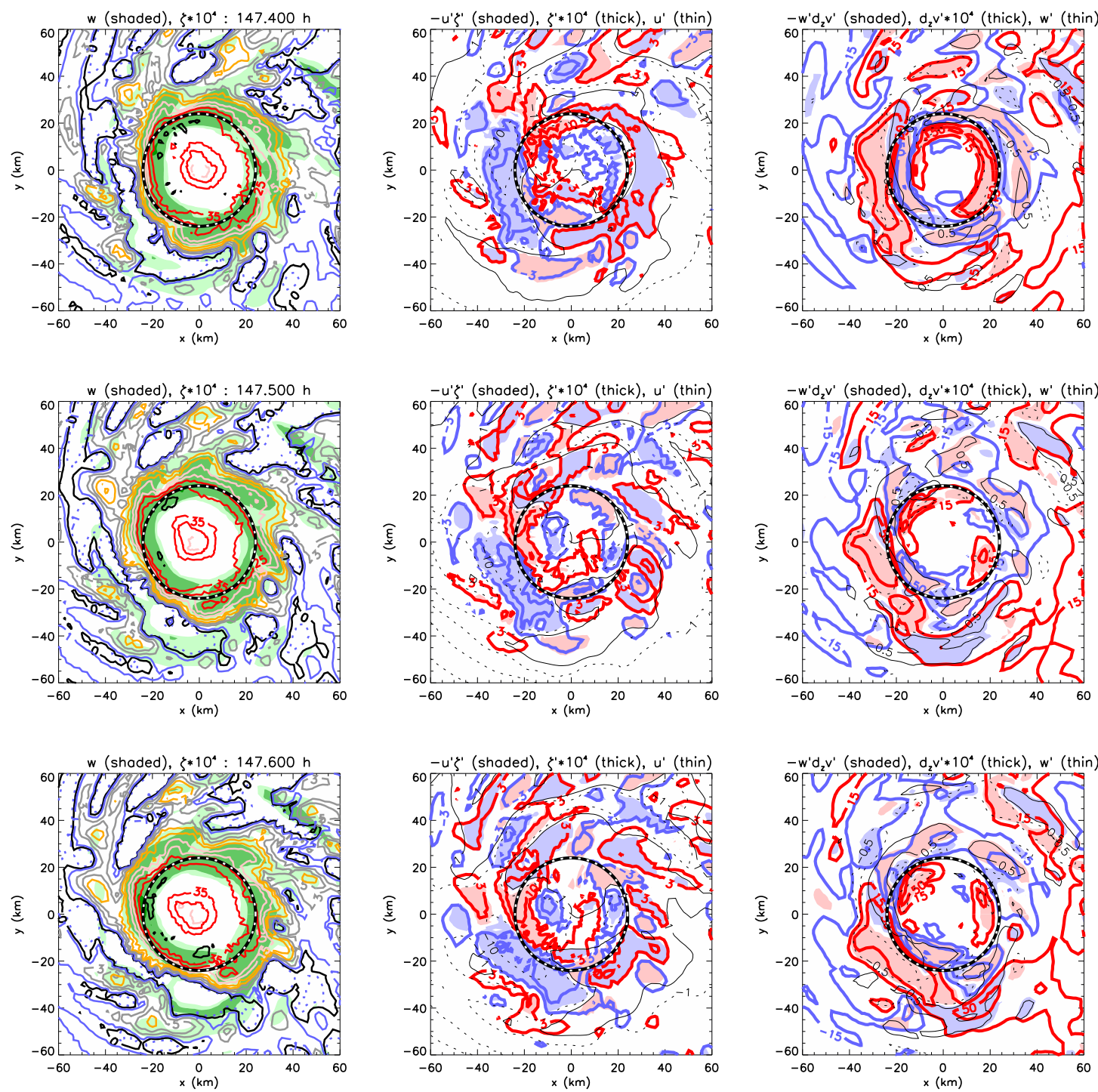

Fig. 20. (Left) Vertical velocity $w$ (shaded; levels $0.3,1.0,3.0 \mathrm{~m} \mathrm{~s}^{-1}$ ) and relative vertical vorticity $\zeta$ times $10^{4}$ (levels ( $\mathrm{s}^{-1}$ ): -1 blue dotted, 0 black, 1 blue, 3 and 5 gray, 7 and 10 orange, 15 and 20 pink, 25 and 35 red, 50 and 75 black) at a sequence of times at $z=1.5 \mathrm{~km}$ from the 3D3k simulation. (Centre) Radial eddy contributions (shaded $\left(\mathrm{m} \mathrm{s}^{-1} \mathrm{~h}^{-1}\right)$ : blue $-u^{\prime} \zeta^{\prime}<-3$; pink $-u^{\prime} \zeta^{\prime}>3$ ) to the azimuthally averaged tangential wind tendency $\partial\langle v\rangle / \partial t$ at the same sequence of times at $z=1.5 \mathrm{~km}$ from the 3D3k simulation. The eddy vertical vorticity $\zeta^{\prime} \times 10^{4}$ (thick blue contours $-30,-10,-3 \mathrm{~s}^{-1}$; thick red contours $3,10,30 \mathrm{~s}^{-1}$ ) and eddy radial velocity $u^{\prime}$ (dashed negative contours: $-10,-1 \mathrm{~m} \mathrm{~s}^{-1}$; thin black contours: $1,10 \mathrm{~m} \mathrm{~s}^{-1}$ ) are superposed. (Right) Vertical eddy contributions $\left(\right.$ shaded (m s $\mathrm{s}^{-1} \mathrm{~h}^{-1}$ ): blue $-w^{\prime} \partial v^{\prime} / \partial z<-3$; pink $\left.-w^{\prime} \partial v^{\prime} / \partial z>3\right)$ to $\partial\langle v\rangle / \partial t$ at the same sequence of times at $z=1.5 \mathrm{~km}$ from the 3D3k simulation. The vertical derivative of eddy tangential wind $\partial v^{\prime} / \partial z \times 10^{4}$ (thick blue contours $-15,-5,-1.5 \mathrm{~s}^{-1}$; thick red contours $1.5,5,15 \mathrm{~s}^{-1}$ ) and eddy vertical velocity $w^{\prime}$ (dashed negative contours $-0.5,-5 \mathrm{~m} \mathrm{~s}^{-1}$; thin black contours $0.5,5 \mathrm{~m} \mathrm{~s}^{-1}$ ). For reference, a spotted-black curve highlights the $r=24 \mathrm{~km}$ radius, the approximate low-level RMW during this analysis interval.

this local Richardson number is small enough to contribute to a nonzero vertical eddy diffusivity in the model is a small subset of the total time series of values ${ }^{17}$. These statements

\footnotetext{
${ }^{17}$ We have verified these conclusions for smaller values of the horizontal and vertical subgrid mixing length parameters: $\left(l_{\mathrm{V}}=20\right.$ $\left.\mathrm{m}, l_{\mathrm{h}}=500 \mathrm{~m}\right)$. In this case, we find no more than $10 \%$ realizations of sub-critical Richardson number in the azimuth-time domain
}

are consistent with plots of the corresponding mean vertical eddy diffusivity during spin-up (Figs. $14 \mathrm{k}$ and $15 \mathrm{k}$ ). The mean diffusivity in the upper eyewall and outflow regions

and the 3-D vortex intensifies in essentially the same manner as the pathway shown here. Further simulations where vertical diffusion is completely suppressed above $1 \mathrm{~km}$ height are essentially similar to the results shown here. 
has a maximum between 8 and $20 \mathrm{~m}^{2} \mathrm{~s}^{-1}$ during the first and second intensification periods, respectively, and is relatively small in comparison to the boundary layer values. Although regions do emerge at the top of the eyewall and top and bottom of the upper-level outflow layer during the mature stage with gradient Richardson numbers near 3, only a thin and radially confined pancake-like region at the bottom of the upper-level outflow possesses gradient Richardson numbers less than unity.

The foregoing pattern of the gradient Richardson number and the lack of strong vertical mixing at upper-levels in the 3-D configuration stand in contrast to those found in the AX configuration (cf. Figs. 17i and 18i). During maturity, the regions of gradient Richardson number in the range between 1 and 3 in the upper-level eyewall and outflow layer are more extensive than in the 3-D model. Moreover, the vertical diffusivity in this region is larger-in-value and the large values cover a larger range of radii than that in the 3-D model.

We conclude that for the realistic model set up used here, the 3-D configuration during spin-up is generally far from criticality with correspondingly little vertical mixing in the upper-level outflow region; only marginal criticality is found during maturity. Thus there is little support for the selfstratification hypothesis in the 3-D model. Our conclusion is consistent with a comment by Emanuel and Rotunno (2011, p. 2245), who say that "we would not expect that the temperature stratification is set by a critical Richardson number criterion when there is little mixing".

\section{The role of frictional drag}

Recent work has highlighted the need to understand further the influence of the boundary layer on vortex intensification (Smith and Vogl, 2008; Smith et al., 2009, 2011; Smith and Thomsen, 2010; Montgomery et al., 2010; Smith and Montgomery, 2010; Nolan et al., 2009a, b). In particular, Montgomery et al. (2010) conducted idealized three-dimensional numerical simulations to investigate the sensitivity of tropical cyclone intensification to changes in the surface drag coefficient in the prototype intensification problem discussed in Sect. 1.4. Changing the drag coefficient provides insight into unbalanced effects in the boundary layer and their impact on the vortex evolution. It provides also further understanding of the intrinsic dependencies of the intensification process on the vertical eddy diffusivity and on the surface drag. The Montgomery et al. (2010) study found that, unlike the predictions of previous work using axisymmetric theory and axisymmetric numerical models, the vortex intensification rate and vortex intensity (up to 4 days) increases with increasing $C_{\mathrm{D}}$ up to approximately $2 \times 10^{-3}$. When $C_{\mathrm{D}}$ is increased further, no significant difference in the intensification rate or intensity occurs until a threshold of approximately $1.3 \times 10^{-2}$, beyond which the intensity decreases. Although the latter drag coefficient is certainly not realistic over the open ocean, the findings suggest nonetheless the relative insensitivity of the intensification rate and mature intensity on meteorologically relevant intensity forecast times scales for drag coefficients typical of high wind speeds over the ocean (Powell et al., 2003; Donelan et al., 2004; Black et al., 2007). By relative insensitivity we mean variations that lie within the predictability envelope for intensity associated with the convective structures that operate in and around the eyewall region of the storm (Nguyen et al., 2008).

While it may be objected that the use of a constant drag coefficient is unrealistic and that the results may be prejudiced by the choice of the bulk-aerodynamic boundary layer scheme in the MM5 model, Smith et al. (2013b) carried out simulations with a more realistic formulation of the drag coefficient and a range of boundary-layer parameterization schemes and showed that the results and supporting interpretations presented by Montgomery et al. (2010) are robust ${ }^{18}$. The implication is that there is a quantitative difference in the role of the frictional boundary layer in the 3-D and AX configurations. Indeed, it will be shown below that this difference is very striking in these configurations. This difference is a further challenge to the notion that the axisymmetric model, as traditionally configured with explicit convection occurring as concentric rings, is a satisfactory approximation for understanding tropical cyclone physics in the prototype problem on meteorologically relevant forecast timescales of 4 to $5 \mathrm{~d}$ and extending out to the long range forecast timescales of $12 \mathrm{~d}$.

For the foregoing reasons a series of simulations is performed here using both the AX and 3-D models to examine the sensitivity of intensification and mature intensity to values of the drag coefficient $C_{\mathrm{D}}$. Recall that in the AX and 3$\mathrm{D}$ control experiments, $C_{k}=1.29 \times 10^{-3}$ and $C_{\mathrm{D}}=2 \times C_{k}$. The additional calculations are carried out using values of the drag coefficient that are multiples of the baseline enthalpy transfer coefficient $C_{k}: C_{\mathrm{D}}=(0.5,1.0,4.0) \times C_{k}$. The enthalpy transfer coefficient is held constant, and a multiple

\footnotetext{
${ }^{18}$ In a very recent paper, the results found by Montgomery et al. (2010) were criticized by Bryan (2013) on the grounds that the calculations were not run long enough to achieve a steady-state solution in which the vortex intensity is at its absolute maximum value. As discussed in Smith et al. (2012, 2013a) and in footnote 9, we believe it is questionable whether a steady-state solution exists. Nevertheless, the calculations summarized and shown here using the CM1 model are for a significantly longer time period (12 d, the same length of time as most simulations published in Bryan, 2013) and more than sufficient to address the intensification and mature phase for realistic forecast timescales. Our findings support further the results and interpretations of Montgomery et al. (2010) and Smith et al. (2012) for this problem posing that is not focused exclusively on the absolute maximum steady-state intensity at some long time ( $>12 \mathrm{~d}$ ). In particular, the 3-D results to be shown here are counter examples to the implied conclusion of Bryan (2013) that the maximum intensity for realistic forecast timescales is inversely proportional to $C_{\mathrm{D}}$ (see Fig. 21a).
} 


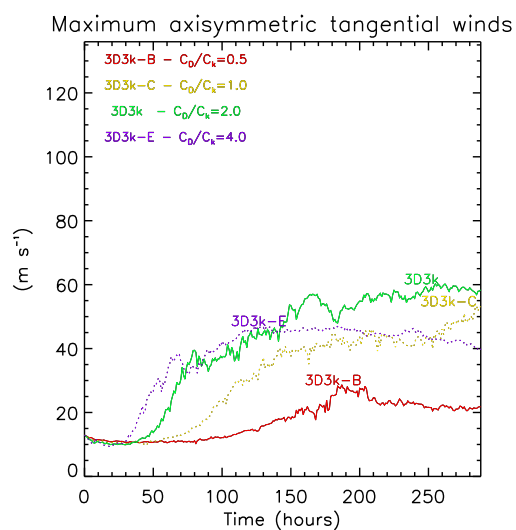

(a)

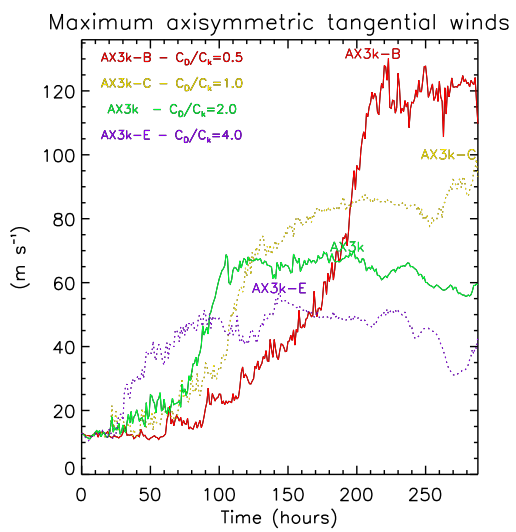

(b)

Fig. 21. Maximum tangential velocity as a function of time for a series of experiments in the CM1 model with increasing surface drag coefficient $C_{\mathrm{D}}$ : (a) four 3-D simulations (3D3k-B, solid red; 3D3k-C, dotted yellow; 3D3k, solid green; 3D3k-E, dotted-blue,), with 3D3k being the control; and (b) four AX simulations (AX3k-B, solid red; AX3k-C, dotted yellow; AX3k, solid green; AX3k-E, dotted blue), with Ax3k being the control. Experiments B have $C_{\mathrm{D}}=0.25 \times$ control $C_{\mathrm{D}}$. Similarly, experiments $(\mathrm{C}, \mathrm{E})$ have $C_{\mathrm{D}}=(0.5,2.0) \times$ control $C_{\mathrm{D}}$, respectively. See text for details.

of 2.0 for the drag coefficient corresponds to the control experiment. The four 3-D simulations, in order of increasing $C_{\mathrm{D}}$, are referred to as "3D3k-B", "3D3k-C", "3D3k", and "3D3k-E", with 3D3k being the control. Likewise, the four $\mathrm{AX}$ simulations, in order of increasing $C_{\mathrm{D}}$, are referred to as "AX3k-B", "AX3k-C", "AX3k", and "AX3k-E", with the AX3k being the control.

The time series of maximum azimuthally averaged tangential velocity are shown in Fig. 21. For both the 3-D and AX configurations, simulations with a large drag coefficient (e.g., blue dotted lines) intensify sooner than those with a small drag coefficient (e.g., red solid lines). In the AX simulations, the mature state intensity appears to be strongly dependent on the value of $C_{\mathrm{D}}$, with the vortex in the lowest drag simulation being much more intense than that in the strongest drag simulation (i.e., $120 \mathrm{~m} \mathrm{~s}^{-1}$ compared with $55 \mathrm{~m} \mathrm{~s}^{-1}$ !). However, in the 3-D configuration, the most intense vortex over the standard forecast timescale of 4 to $5 \mathrm{~d}$ occurs with a moderately strong (and realistic) level of surface drag (our control configuration).

With the range of variability of maximum intensity found by Montgomery et al. (2010), Smith et al. (2013b) and Thomsen et al. (2013) using the MM5 model, the range shown by the control and 3D $3 \mathrm{k}-\mathrm{E}$ simulations up to $5 \mathrm{~d}$ lie close to the range of the variability of the 3-D simulations shown earlier in Fig. 1. In this sense, the control and double-thecontrol drag simulations do not predict a significantly different mature intensity for a $5 \mathrm{~d}$ simulation time, broadly consistent with the foregoing studies. On the timescale of 4-5 $\mathrm{d}$, the vortex in the half-the-control drag simulation, 3D3k$\mathrm{C}$, is again weaker than that in the control simulation. At longer times, however, the vortex in the 3D3k-C simulation "catches up" to the slowly intensifying vortex in the control simulation, while the vortex in the double-the-control drag simulation, 3D3k-E, stays at approximately the same intensity. At very long times $(\sim 10 \mathrm{~d})$ vortices in both the $3 \mathrm{D} 3 \mathrm{k}$ and 3D3k-E simulations weaken with time. By $12 \mathrm{~d}$, the vortex in the strongest drag simulation is about $20 \mathrm{~m} \mathrm{~s}^{-1}$ weaker than that in the control configuration. However, in the weakest drag simulation, 3D3k-B, the vortex struggles to intensify throughout the entire $12 \mathrm{~d}$ integration time. Needless to say, the $3 \mathrm{D} 3 \mathrm{k}-\mathrm{B}$ solution is considerably weaker than that of the corresponding AX3k-B solution after approximately $210 \mathrm{~h}(8.75 \mathrm{~d})\left(29 \mathrm{~m} \mathrm{~s}^{-1} \text { versus } 120 \mathrm{~m} \mathrm{~s}^{-1} \text { !) }\right)^{19}$.

The interpretations given in Smith et al. (2013b) for the vortex behaviour as a result of changing the boundary-layer scheme, or changing the drag coefficient within a boundarylayer scheme, go some way to providing basic understanding of the issues discussed above. However, they fall short of providing a complete theory, which would require consideration of processes above the boundary layer as well as in the boundary layer. In particular, the magnitude and radial distribution of the heating rate may change as the drag coefficient changes. While the lack of such a theory presents an obstacle to interpreting the behaviour shown in Fig. 21 in both the 3-D and AX configurations, we attempt to articulate some of the key elements required to elucidate these differences.

To explain the behaviour of the 3-D model to changes in $C_{\mathrm{D}}$, Smith et al. (2013b) invoked the frictional disruption of gradient wind balance in the boundary layer, which increases with $C_{\mathrm{D}}$. Their arguments were applied to the azimuthally averaged flow fields. It was shown in Montgomery

\footnotetext{
${ }^{19}$ Because of the scatter in the observational data used to set the vertical mixing length discussed in Sect. 2, we have conducted an independent numerical experiment and verified the large tangential wind speed obtained in the AX model using a value of $l_{\mathrm{V}}=100 \mathrm{~m}$ instead of $l_{\mathrm{V}}=50 \mathrm{~m}$ (not shown).
} 


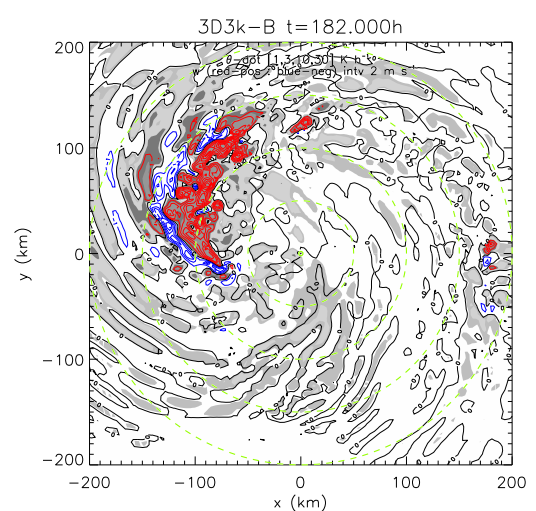

(a)

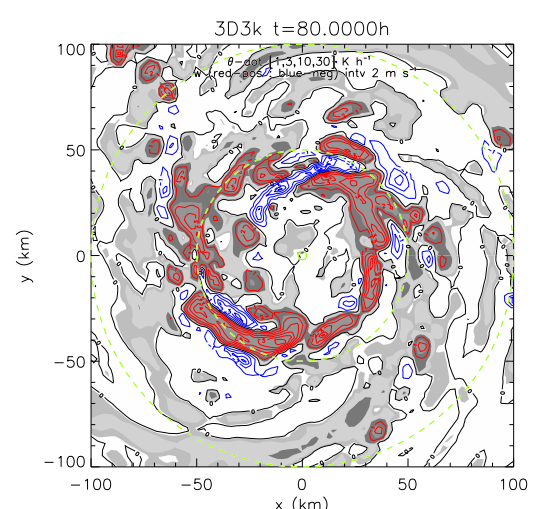

(b)

Fig. 22. Plan-view plots at the $z=5 \mathrm{~km}$ level during rapid intensification for (a) simulation 3D3k-B ("low drag") at $182 \mathrm{~h}$ and (b) simulation 3D3k ('realistic drag') at $80 \mathrm{~h}$ of vertical velocity $w$ with contour interval $2 \mathrm{~m} \mathrm{~s}^{-1}$ (blue negative; red positive) and diabatic heating rate $\dot{\theta}$ with shading levels of $1,3,10$, and $30 \mathrm{~K} \mathrm{~h}^{-1}$. The dashed green rings are constant radii spaced at $50 \mathrm{~km}$ from storm centre.

et al. (2010) and Smith et al. (2013b) that a decrease in $C_{\mathrm{D}}$ leads to a weaker inflow in the boundary layer, to a smaller inward displacement of $\langle M\rangle$ surfaces, and therefore lower tangential wind speeds in the mature vortex. This argument would explain why a decrease in $C_{\mathrm{D}}$ in both models leads to a slower spin-up at early times. Nevertheless, unlike the behaviour in the 3-D simulation, a stronger mature vortex arises in the AX simulation when the drag coefficient is reduced. Since the above arguments relate to the azimuthally averaged flow, why then do they apparently fail to explain the radically different behaviour between the AX and 3-D model as the mature stage is approached? Implicit in the foregoing explanation is the presumption that the magnitude and radial distribution of the azimuthally averaged heating rate do not change appreciably. However, we will show below that this is not the case and that the mean heating rate is much weaker in the reduced drag simulations for the 3-D model. We consider this issue further in the remainder of this section.

Recall from Sect. 3 that the relatively large heating rates in the AX model are an artifice of the fact that convection is ring-like. However, until an eyewall feature has formed, the more realistic convection simulated in the 3-D model has a limited azimuthal scale so that azimuthally averaged heating rates are much less than in the AX model. We demonstrate below that the frictional boundary layer is especially important in the 3-D configurations in organizing the random distribution of convection into a quasi ring-like structure. In turn, a ring-like convective structure is conducive to generating azimuthally coherent convergence that would appear to be broadly advantageous for vortex spin-up by helping sustain low convective inhibition. In the AX configurations the convection is already ring-like and doesn't require any azimuthal organization.

The dependence of the convective organization on the drag coefficient $C_{\mathrm{D}}$ is succinctly illustrated in Fig. 22 for two of the 3-D experiments of Fig. 18. The figure overlays the di- abatic heating/cooling rate with the vertical motion field for the "low drag" simulation (3D3k-B) and the "realistic" drag simulation (3D3k) at the time of most rapid intensification of the mean tangential wind in relation to the low-level circulation centre as defined in Sect. 4 (open green circle). For the low-drag simulation, it is clear that the convection is maximized at a relatively large radius from the circulation centre and is highly confined azimuthally to the upper left quadrant. In contrast, in the realistic-drag simulation the convection is more organized at smaller radii near the centre of circulation and is more evenly distributed in azimuth (see Fig. 22b). These two examples lend strong support to the idea that as the drag coefficient is increased from small to realistic values, surface friction significantly fosters convective organization in the 3-D model. This effect is not present in the AX model.

As noted above, the arguments concerning the role of friction in the boundary layer between the low drag and realistic drag simulations assume that the magnitude and radial distribution of the azimuthally averaged heating rate do not change appreciably. In fact, the time-radius plot of Fig. 23b shows that the azimuthally averaged heating rate at a height of $6 \mathrm{~km}$ in the 3-D simulation with reduced drag is appreciably less than that in the standard drag configuration, Fig. 23a. This difference has a significant impact on the radial displacement of the $\langle M\rangle$ surfaces during the vortex evolution and, in particular, the ability to bring some $\langle M\rangle$ surfaces to small radii. In Fig. 23, the $\langle M\rangle$ surfaces are shown at a height of $1 \mathrm{~km}$, a height that is slightly above the height of maximum tangential wind during the evolution. For reference, Fig. 23 displays also the time evolution of the RMW and that of the $\langle M\rangle$ contour coinciding with the RMW in the mature stage.

For the case of standard drag (panel a), the $\langle M\rangle$ surfaces begin to move inwards when the heating rate becomes appreciable in the inner region. In particular, the $\langle M\rangle$ surface that marks the RMW at $1 \mathrm{~km}$ height in the mature stage starts at 


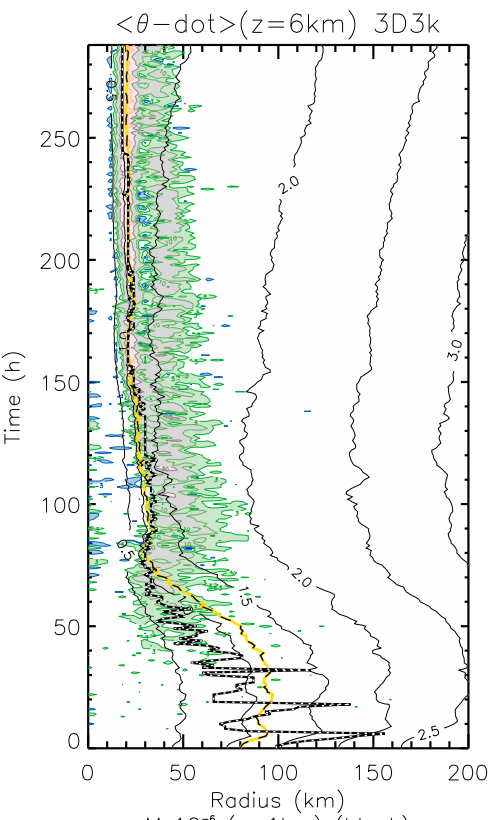

(a)

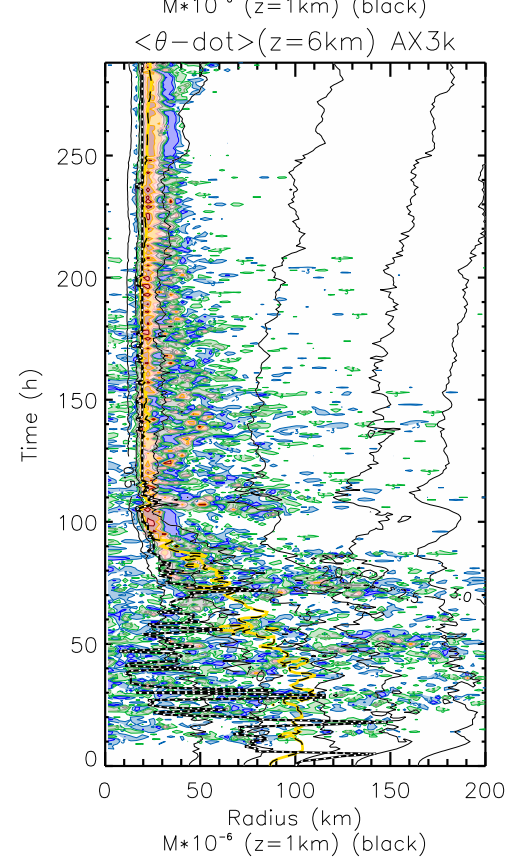

(b)

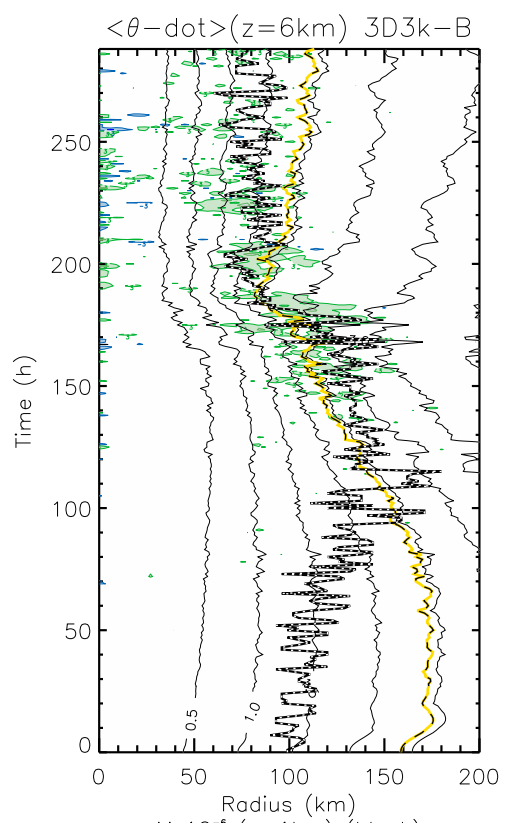

$M * 10^{-6}(z=1 \mathrm{~km})$ (black)

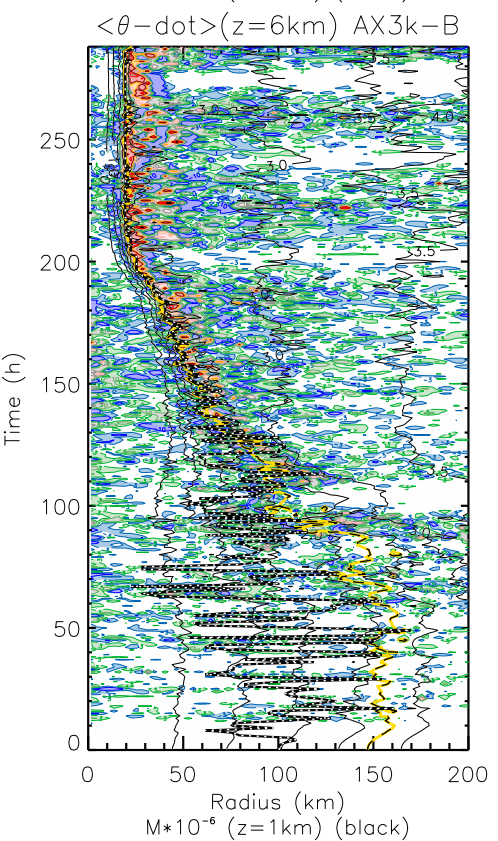

Fig. 23. Azimuthal mean heating rate $(\langle\dot{\theta}\rangle$, shaded) and azimuthal mean absolute angular momentum $(\langle M\rangle$, black contours, interval $0.5 \times$ $10^{6} \mathrm{~m}^{2} \mathrm{~s}^{-1}$ ) from the (a) 3D3k, (b) 3D3k-B, (c) AX3k, and (d) AX3k-B simulations. Colored $\langle\dot{\theta}\rangle$ contours $\left(\mathrm{K} \mathrm{h}^{-1}\right)$ are $-10($ dark blue), -3 (blue), 3 (green), 10 (light green), 30 (gray), 50 (pink), 75 (orange), 100 (red), 150 (red), and 200 (black). The white-spotted black line is the RMW. The yellow-dashed black line is the value of $\langle M\rangle$ found at the RMW at times of mature intensity for each simulation.

an initial radius of $80 \mathrm{~km}$ and moves in to a small RMW of about $20 \mathrm{~km}$. In contrast, the corresponding $\langle M\rangle$ surface in the reduced drag case (panel b) begins at a radius of $160 \mathrm{~km}$ and moves in only to a radius of $80 \mathrm{~km}$. However, for this case the arguments in terms of $\langle M\rangle$ surfaces are less appropriate because the vortex is highly asymmetric even in the mature stage (see Fig. 22a), a situation in which the simple explanation of the approximate material conservation of the azimuthally averaged $M$ is not expected.

The situation in the AX simulations is dramatically different in several ways. First, consistent with the findings of Sect. 3, the heating rates in the AX simulations are significantly larger in magnitude than in the 3-D simulations. Second, the heating rate at $6 \mathrm{~km}$ height in the AX simulation with 
reduced drag (Fig. 23d) is appreciably larger than for the AX simulation with standard drag (Fig. 23c). Third, in both AX simulations, the heating rate exhibits considerably more variability than that in the 3-D simulations. Fourth, in both AX simulation at early times, the RMW shows large radial fluctuations (sometimes more than $100 \mathrm{~km}$ ) and these fluctuations persist for a longer period in the low drag simulation. Fifth, in the low drag simulation, large values of heating rate extend to significant radii $(\sim 200 \mathrm{~km})$ for much of the simulation.

In the AX simulations, the $\langle M\rangle$ surface that marks the RMW has an initial radius of $150 \mathrm{~km}$ in the case of reduced drag compared with $90 \mathrm{~km}$ in the case of standard drag, although the final radius is about the same in both cases shown in Fig. $23(\sim 20 \mathrm{~km})$. The time taken for the minimum RMW to be achieved is significantly greater in the case of reduced drag $(\sim 200 \mathrm{~h})$ compared to that of standard drag $(\sim 100 \mathrm{~h})$. As discussed above, the increased boundary-layer convergence associated with an increase in $C_{\mathrm{D}}$ would help to explain the faster spin-up in the AX configuration. However, in the later stages of vortex evolution, the much larger heating rates in the low drag simulation have generated a larger inward displacement of the $\langle M\rangle$ surface which corresponds to the RMW and therefore a much stronger vortex. Recalling the application of the spin-up function in Sect. 4 (and references cited therein), the existence of convection over a broader range of radii in the low drag simulation would be another factor in explaining the slower development rate in this case.

The foregoing discussion goes some way to providing an explanation as to why the behaviour of the 3-D and AX simulations to a decrease in the drag coefficient is so radically different. Below we offer some further elements of an explanation for these differences in the context of azimuthally averaged dynamics.

In terms of strictly axisymmetric boundary-layer dynamics, an increase of $C_{\mathrm{D}}$ in the radial momentum equation will lead to a larger inward agradient force, but also to a larger outward frictional drag on the inflow. Whether or not the inflow increases with increasing $C_{\mathrm{D}}$ depends on which of these effects dominates. If the inflow increases, so will the inward advection of $\langle M\rangle$ surfaces. However, if the drag increases, $\langle M\rangle$ will be lost at a greater rate because of the increased frictional torque. One has to do the calculation to determine which effect dominates in the radial and tangential momentum equations. Because of the quadratic increase of drag with wind speed, one would expect that these effects to be intensity dependent and the outcome could be different between the 3-D and AX calculations. The calculations summarized in Fig. 21 suggest that during the early part of the intensification phase in both 3-D and AX models, an increase in $C_{\mathrm{D}}$ leads to stronger inflow and the accompanying increase in the inward advection of $\langle M\rangle$ exceeds the frictional depletion of $\langle M\rangle$, leading to a faster spin-up. For reasons discussed above, the foregoing discussion may be less applicable to the lowest drag case in the 3-D configuration.
The difficulty of anticipating a priori which of the foregoing effects dominates is compounded by the coupling of the boundary-layer dynamics and thermodynamics to the flow above, particularly the azimuthally averaged radial and vertical distribution of the diabatic heating rate. Based on the results shown in Sect. 6, the eddy fluxes of momentum may play an important role also. The final outcome on the mature intensity depends in principal on all of these factors. At this point, we are unable to foresee the outcome without performing explicit calculations.

\section{Conclusions}

We have carried out idealized numerical experiments to examine the differences between tropical cyclone evolution in three-dimensional and axisymmetric configurations for the prototype intensification problem. The choice of subgridscale horizontal and vertical mixing length parameters, as well as air-sea momentum and enthalpy exchange coefficients, is guided by recent observational work.

This study has identified a number of important differences between the two configurations. Many of these differences may be attributed to the dissimilarity of deep cumulus convection in the two models. For example, there are fundamental differences in convective organization. Deep convection in the three-dimensional model is sheared tangentially by the differential angular rotation of the system-scale circulation in the radial and vertical directions, unlike that in the axisymmetric configuration. Because convection is not organized into concentric rings during the spin-up process, the azimuthally averaged heating rate and radial gradient thereof is considerably less than that in the axisymmetric model. For most of the time this lack of organization results in slower spin-up and leads ultimately to a weaker mature vortex. There is a short period of time, however, when the rate of spin-up in the 3-D model exceeds that of the maximum spin-up rate in the AX model. During this period the convection is locally more intense than in the axisymmetric model and the convection is organized in a quasi ring-like structure resembling a developing eyewall. These regions of relatively strong updraughts have an associated vertical eddy momentum flux that contribute significantly to the spin-up of the azimuthal mean vortex and provide an explanation for the enhanced spin-up rate in the 3-D model despite the relative weakness of the azimuthal mean heating rate and its radial derivative in the 3-D model.

Consistent with findings of previous work, the mature intensity in the 3-D model is lower than that in the AX model.

In contrast with previous interpretations invoking barotropic instability and related mixing processes as a mechanism detrimental to the spin-up process, the results herein suggest that eddy processes associated with vortical plume structures can assist the intensification process via up-gradient momentum fluxes in the radial direction. These 
plumes contribute also to the azimuthally averaged heating rate and the corresponding azimuthal-mean overturning circulation. Our analysis has unveiled a potentially important issue in the representation of subgrid scale parameterizations of eddy momentum fluxes in hurricane models. Comparisons between the two model configurations indicate that the structure of the resolved eddy momentum fluxes above the boundary layer differs from that prescribed by the subgridscale parameterizations in either the three-dimensional or axisymmetric configurations, with the exception perhaps of the resolved horizontal eddy momentum flux during the mature stages.

Another important difference between the two configurations is that the flow fields in the axisymmetric model tend to be much noisier than in the three-dimensional model. The larger flow variability is because the deep convection generates azimuthally coherent, large-amplitude, inertia-gravity waves. Although deep convection in the three-dimensional model generates inertia-gravity waves also, the convection is typically confined to small ranges of azimuth and tends to be strained by the azimuthal shear. These effects lead to a reduced amplitude of variability in the azimuthally averaged flow fields.

An analysis of the spin-up function was carried out to determine the extent to which the conventional spin-up mechanism, discussed in the Introduction, provides a useful interpretation for the intensification of the bulk vortex. In the 3-D model, a coherent region with positive values of spin-up function is found just inside the radius of maximum tangential wind and extends vertically in the main eyewall region in association with the peak eyewall heating rate near $7 \mathrm{~km}$ altitude, the signal identified by Vigh and Schubert (2009) for intensification. This region is unlike that found at larger radii, which is dominated by larger-in-amplitude, but smaller-in scale positive and negative dipole structures that would tend to be filtered in a balanced inversion of the geopotential tendency equation. The results for the 3 -D model show that during spin-up, the time variation of the volume-averaged gradient wind tendency inside the radius of maximum gradient wind mimics closely that of the volume-averaged spinup function. Although a correspondence between these two quantities persists in the AX model during the primary intensification period (with the spin-up function approximately double that of the corresponding 3-D simulation), the higher degree of variability associated with inertia-gravity waves is a pervading feature, even inside the RMW, and at later times compounds the interpretation of the spin-up in this model in terms of balance dynamics.

An analysis of terms in the tangential momentum equation showed that the spin-up of the azimuthally averaged maximum tangential wind speed in both models takes place within the frictional boundary layer confirming recent predictions and observations. In the 3-D model, surface drag plays a particularly important role in the spin-up process by tending to organize the convection in azimuth. There is a radical dif-
Table A1. Vertical grid mesh $(\mathrm{km})$.

\begin{tabular}{llll}
\hline 0.025 & 0.090 & 0.184 & 0.308 \\
0.461 & 0.644 & 0.856 & 1.097 \\
1.369 & 1.669 & 1.999 & 2.359 \\
2.748 & 3.167 & 3.615 & 4.092 \\
4.599 & 5.136 & 5.702 & 6.297 \\
6.922 & 7.577 & 8.261 & 8.974 \\
9.717 & 10.49 & 11.29 & 12.12 \\
12.98 & 13.87 & 14.79 & 15.74 \\
16.72 & 17.73 & 18.77 & 19.84 \\
20.93 & 22.06 & 23.21 & 24.40 \\
\hline
\end{tabular}

ference in behaviour of the 3-D and AX models when the surface drag is reduced or increased from realistic values. Borrowing from ideas developed in a recent paper, we have sought to give a partial explanation for this difference in behaviour.

Analyses of the gradient Richardson number in the threedimensional model do not support a recent hypothesis concerning the role of small-scale vertical mixing processes in the upper-tropospheric outflow in controlling the spin-up process.

Our results provide new qualitative and quantitative insight into the asymmetric and symmetric dynamics of tropical cyclones and we believe they are relevant to the formulation of a more satisfactory theory of tropical cyclone intensification and mature intensity. In particular, the results point to some fundamental limitations of strict axisymmetric theory and modeling for representing the azimuthally averaged behaviour of tropical cyclones in three dimensions. Specifically, the results herein suggest that the representation of convection as concentric rings in the AX model and the assumption of down-gradient eddy momentum fluxes to represent asymmetric eddy transport processes in both 3-D and AX models are flaws in the formulation and interpretation of tropical cyclone models.

\section{Appendix A}

In the three-dimensional simulations, the $x$ and $y$ coordinates are treated identically with a stretched grid configuration. Throughout the inner-most $405 \mathrm{~km}$, there is a fixed grid spacing of $3 \mathrm{~km}$, which is then stretched gradually to a grid spacing of $97.98 \mathrm{~km}$ at the outer edge of the domain. The entire domain spans $2880 \mathrm{~km}$ with 185 grid points. The vertical coordinate is stretched also with a grid spacing of $50 \mathrm{~m}$ near the surface and one of $1200 \mathrm{~m}$ near the domain top at $25 \mathrm{~km}$. The lowest grid level where horizontal winds and thermodynamic variables are defined is at $25 \mathrm{~m}$. The entire vertical domain is spanned with 40 grid points (Table 1). The total number of computational points is therefore $185 \times 185 \times 40=1369000$ grid points. The number of computational points in the uniform inner-grid domain is 
$136 \times 136 \times 40=739840$ grid points. The axisymmetric simulations employ a fixed radial grid spacing of $3 \mathrm{~km}$, but use the same stretched vertical grid as described above.

Acknowledgements. MTM and JP acknowledge financial support from NSF ATM-0733380 and the U.S. Office of Naval Research Grant No. N00014-03-1-0185. We wish to acknowledge also the computational resources of M. Kirby for supplemental simulations.

Edited by: T. J. Dunkerton

\section{References}

Anthes, R. A.: Tropical cyclones: Their evolution, structure, and effects. Meteor. Monogro., No. 41, Amer. Meteor. Soc., 208 pp., 1982.

Anthes, R. A., Trout, J. W., and Rosenthal, S. L.: Comparisons of tropical cyclone simulations with and without the assumption of circular symmetry, Mon. Weather Rev., 99, 759-766, 1971.

Bell, M. M. and Montgomery, M. T.: Sheared deep vortical convection in pre-depression Hagupit during TC S08, Geophys. Res. Lett., 37, L06802, doi:10.1029/2009GL042313, 2010.

Bell, M. M., Montgomery, M. T., and Lee, W.-C.: An axisymmetric view of concentric eyewall evolution in Hurricane Rita (2005), J. Atmos. Sci., 69, 2414-2432, 2012.

Bister, M. and Emanuel, K. A.: Dissipative heating and hurricane intensity, Meteor. Atm. Phys., 52, 233-240, 1998.

Black, P. G., D’Asaro, E. A., Sanford, T. B., Drennan, W. M., Zhang, J. A., French, J. R., Niiler, P. P., Terrill, E. J., and Walsh, E. J.: Air-sea exchange in hurricanes: Synthesis of observations from the coupled boundary layer air-sea transfer experiment, B. Am. Meteorol. Soc., 88, 357-374, 2007.

Braun, S., Montgomery, M., Mallen, K., and Reasor, P.: Simulation and interpretation of the genesis of tropical storm Gert (2005) as part of the NASA Tropical Cloud System and Processes Experiment, J. Atmos. Sci., 67, 999-1025, 2010.

Brown, B. R. and Hakim, G. J.: Variability and predictability of a three-dimensional hurricane, J. Atmos. Sci., 70, 1806-1820, 2013.

Bryan, G. H.: Effects of surface exchange coefficient and turbulence length scales on the intensity and structure of numerically simulated hurricanes, Mon. Weather Rev., 140, 1125-1143, 2012.

Bryan, G. H.: Comments on "Sensitivity of tropical-cyclone models to the surface drag coefficient", Q. J. R. Meteorol. Soc., 139, 1957-1960, 2013.

Bryan, G. H. and Fritsch, J. M.: A benchmark simulation for moist nonhydrostatic numerical model, Mon. Weather Rev., 130, $2917-$ 2928, 2002.

Bryan, G. H. and Rotunno, R.: The influence of near-surface, highentropy air in hurricane eyes on maximum hurricane intensity, J. Atmos. Sci., 66, 148-158, 2009a.

Bryan, G. H. and Rotunno, R.: Evaluation of an analytical model for the maximum intensity of tropical cyclones, J. Atmos. Sci., 66, 3042-3060, 2009b.

Bryan, G. H., Rotunno, R., and Chen, Y.: The effects of turbulence on hurricane intensity. 29th Conference on Hurricanes and Tropical Meteorology, Amer. Meteor. Soc., Tucson, AZ, 8C.7, 2010.
Bui, H. H., Smith, R. K., Montgomery, M. T., and Peng, J.: Balance and unbalanced aspects of tropical cyclone intensification, Q. J. R. Meteorol. Soc., 135, 1715-1731, 2009.

Camp, J. P. and Montgomery, M. T.: Hurricane maximum intensity: Past and present, Mon. Weather Rev., 129, 1704-1717, 2001.

Carr III, L. E. and Williams, R. T.: Barotropic vortex stability to perturbations from axisymmetry, J. Atmos. Sci., 46, 3177-3191, 1989.

Carrier, G. F.: The intensification of hurricanes, J. Fluid Mech., 49, 145-158, 1971.

Carrier, G. F., Hammond, A. L., and George, O. D.: A model of the mature hurricane, J. Fluid Mech., 47, 145-170, 1971.

Chen, Y., and Yau, M. K.: Spiral bands in a simulated hurricane. Part I: Vortex Rossby wave verification, J. Atmos. Sci., 58, 21282145, 2001.

Chen, Y., Brunet, G., and Yau, M. K.: Spiral bands in a simulated hurricane. Part II: Wave activity diagnostics, J. Atmos. Sci., 60, 1239-1256, 2003.

Donelan, M. A., Haus, B. K., Reul, N., Plant, W. J., Stiassne, M., Graber, H. C., Brown, O. B., and Saltman, E. S.: On the limiting aerodynamic roughness in very strong winds, Geophys. Res. Lett., 31, L18306, doi:10.1029/2004GL019460, 2004.

Dunkerton, T. J., Montgomery, M. T., and Wang, Z.: Tropical cyclogenesis in a tropical wave critical layer: easterly waves, Atmos. Chem. Phys., 9, 5587-5646, doi:10.5194/acp-9-5587-2009, 2009.

Durran, D. R. and Klemp, J. B.: A compressible model for the simulation of moist mountain waves, Mon. Weather Rev., 111, 23412361, 1983.

Emanuel, K. A.: An air-sea interaction theory for tropical cyclones. Part I: Steady state maintenance, J. Atmos. Sci., 43, 585-604, 1986.

Emanuel, K. A.: The finite amplitude nature of tropical cyclogenesis, J. Atmos. Sci., 46, 3431-3456, 1989.

Emanuel, K. A.: The theory of hurricanes. Ann. Rev. Fluid Mech., 23, 179-196, 1991

Emanuel, K. A.: Sensitivity of tropical cyclones to surface exchange coefficients and a revised steady-state model incorporating eye dynamics, J. Atmos. Sci., 52, 3969-3976, 1995.

Emanuel, K. A.: Some aspects of hurricane inner-core dynamics and energetics, J. Atmos. Sci., 54, 1014-1026, 1997.

Emanuel, K. A.: Thermodynamic control of hurricane intensity, Nature, 401, 665-669, 1999.

Emanuel, K. A.: Tropical cyclone, Annu. Rev. Earth Planet Sci., 31, 75-104, 2003.

Emanuel, K. A.: Self-stratification of tropical cyclone outflow. Part II: Implications for storm intensification, J. Atmos. Sci., 69, 988996, 2012.

Emanuel, K. A. and Rotunno, R.: Self-statification of tropical cyclone outflow. Part I: Implications for storm structure, J. Atmos. Sci., 68, 2236-2249, 2011.

Emanuel, K. A., Neelin, J. D., and Bretherton, C. S.: On large-scale circulations in convecting atmosphere, Q. J. R. Meteorol. Soc., 120, 1111-1143, 1994.

Emanuel, K. A., DesAutels, C., Holloway, C., and Korty, R.: Environmental control of tropical cyclone intensity, J. Atmos. Sci., 61, 843-858, 2004.

Fang, J. and Zhang, F.: Initial development and genesis of Hurricane Dolly (2008), J. Atmos. Sci., 67, 655-672, 2010. 
Gill, A. E.: Atmosphere-Ocean Dynamics (International Geophysics Series, Volume 30), Academic Press. 662 pp., 1982.

Gopalakrishnan, S. G., Marks, F., Jr., Zhang, X., Bao, J.-W., Yeh, K.-S., and Atlas, R.: The experimental HWRF system: A study on the influence of horizontal resolution on the structure and intensity changes in tropical cyclones using an idealized framework, Mon. Weather Rev., 139, 1762-1784, 2011.

Guinn, T. A. and Schubert, W. H.: Hurricane spiral bands, J. Atmos. Sci., 50, 3380-3403, 1993.

Hack, J. J. and Schubert, W. H.: Nonlinear response of atmospheric vortices to heating by organized cumulus convection, J. Atmos. Sci., 43, 1559-1573, 1986.

Hakim, G. J.: The mean state of axisymmetric hurricanes in statistical equilibrium, J. Atmos. Sci., 68, 1364-1376, 2011.

Haus, B. K., Jeong, D., Donelan, M. A., Zhang, J. A., and Savelyev, I.: Relative rate of sea-air heat transfer and frictional drag in very high winds, Geophys. Res. Lett., 37, L07802, doi:10.1029/2009GL042206, 2010.

Haynes, P. and McIntyre, M. E.: On the evolution of vorticity and potential vorticity in the presence of diabatic heating and frictional or other forces, J. Atmos. Sci., 44, 828-841, 1987.

Hendricks, E. A., Montgomery, M. T., and Davis, C. A.: The role of "vortical" hot towers in the formation of tropical cyclone Diana (1984), J. Atmos. Sci., 61, 1209-1232, 2004.

Holton, J. R.: An introduction to dynamic meteorology, Academic Press, London, 535 pp., 2004.

Hoskins, B. J. and Bretherton, F. P.: Atmospheric frotogenesis models: Mathematical forulation and solution, J. Atmos. Sci., 54, 1137, 1972.

Huang, Y.-H., Montgomery, M. T., and Wu, C.-C.: Concentric eyewall formation in Typhoon Sinlaku (2008): Part II: Axisymmetric dynamical processes, J. Atmos. Sci., 69, 662-674, 2012.

Julian, K., Legg, S., McWilliams, J., and Werne, J.: Rapidly rotating turbulent Rayleigh-Bénard convection, J. Fluid Mech., 322, $243-$ 273, 1996.

Kilroy, G. and Smith, R. K.: A numerical study of rotating convection during tropical cyclogenesis, Q. J. R. Metorol. Soc., in press, 2012.

Kurihara, Y. and Bender, M. A.: Structure and analysis of the eye of a numerically simulated tropical cyclone, J. Meteorol. Soc. Japan, 60, 381-395, 1982.

Lilly, D. K.: On the numerical simulation of buoyant convection, Tellus, 14, 148-172, 1962.

Malkus, J. S.: On the structure of the mature hurricane eye, J. Met., 15, 337-349, 1958.

Malkus, J. S. and Riehl, H.: On the dynamics and energy transformations in steady-state hurricanes, Tellus, 12, 1-20, 1960.

Martinez, Y. H.: Diagnostic study of hurricane asymmetries using empirical normal modes, Ph. D. thesis, McGill University, 188 pp., 2008.

Martinez, Y., Brunet, G., and Yau, M. K.: On the dynamics of two-dimensional hurricane-like vortex symmetrization, J. Atmos. Sci., 67, 3559-3580, 2010.

Martinez, Y., Brunet, G., Yau, M. K., and Wang, X.: On the dynamics of concentric eyewall genesis: Space-time empirical normal modes diagnosis, J. Atmos. Sci., 68, 457-476, 2011.

McWilliams, J. C., Graves, L. P., and Montgomery, M. T.: A formal theory for vortex Rossby waves and vortex evolution, Geophys. Astrophys. Fluid Dyn., 97, 275-309, 2003.
Melander, M. V., McWilliams, J. C., and Zabusky, N. J.: Axisymmetrization and vorticity-gradient intensification of an isolated two-dimensional vortex through filamentation, J. Fluid Mech., 178, 137-159, 1987.

Michalke, A. and Timme, A.: On the inviscid instability of certain two-dimensional vortex-type flow. J. Fluid Mech., 29, 647-666, 1967.

Miller, B. I.: On the maximum intensity of hurricanes, J. Met., 15, 184-195, 1958.

Moeng, C.-H., McWilliams, J. C., Rotunno, R., Sullivan, P. P., and Weil, J.: Investigating 2D modeling of atmospheric convection in the PBL, J. Atmos. Sci., 61, 889-903, 2004.

Möller, J. D. and Montgomery, M. T.: Tropical cyclone evolution via potential vorticity anomalies in a three-dimensional balance model, J. Atmos. Sci., 57, 3366-3387, 2000.

Möller, J. D. and Shapiro, L. J.: Balanced contributions to the intensification of Hurricane Opal as diagnosed from a GFDL model forecast, Mon. Weather Rev., 130, 1866-1881, 2002.

Montgomery, M. T. and Enagonio, J.: Tropical cyclogenesis via convectively forced vortex Rossby waves in a three-dimensional quasigeostrophic model, J. Atmos. Sci., 55, 3176-3207, 1998.

Montgomery, M. T. and Kallenbach, R. J.: A theory for vortex Rossby-waves and its application to spiral bands and intensity changes in hurricanes, Q. J. R. Meteor. Soc., 123, 435-465, 1997.

Montgomery, M. T. and Shapiro, L. J.: Generalized Charney-Stern and Fjortoft theorems for rapidly rotating vortices, J. Atmos. Sci., 52, 1829-1833, 1995.

Montgomery M. T. and R. K. Smith: Paradigms for tropical-cyclone intensification, Aust. Meteor. Ocean. Journl., in press, 2013.

Montgomery, M. T., Nicholls, M. E., Cram, T. A., and Saunders, A. B.: A vortical hot tower route to tropical cyclogenesis, J. Atmos. Sci., 63, 355-386, 2006.

Montgomery, M. T., Nguyen, S. V., Persing, J., and Smith, R. K.: Do tropical cyclones intensify by WISHE?, Q. J. R. Meteorol. Soc., 135, 1697-1714, 2009.

Montgomery, M. T., Smith, R. K., and Nguyen, S. V.: Sensitivity of tropical cyclone models to the surface drag coefficient, Q. J. R. Meteorol. Soc., 136, 1945-1953, 2010.

Montgomery, M. T., Davis, C., Dunkerton, T., Wang, Z., Velden, C., Torn, R., Majumdar, S. J., Zhang, F., Smith, R. K., Bosart, L., Bell, M. M., Haase, J. S., Heymsfield, A., Jensen, J., Campos, T., and Boothe, M. A.: The pre-depression investigation of cloud systems in the tropics (PREDICT) experiment: Scientific basis, new analysis tools, and some first results, Bull. Amer. Met. Soc., 93, 153-172, 2012.

Nguyen, C. M., Smith, R. K., Zhu, H., and Ulrich, W.: A minimal axisymmetric hurricane model, Q. J. R. Meteorol. Soc., 128, 2641-2661, 2002.

Nguyen, V. S., Smith, R. K., and Montgomery, M. T.: Tropicalcyclone intensification and predictability in three dimensions, Q. J. R. Meteorol. Soc., 134, 563-582, 2008.

Nguyen C. M., Reeder, M. J., Davidson, N. E., Smith, R. K., and Montgomery, M. T.: Inner-core vacillations cycles during the intensification of Hurricane Katrina, Q. J. R. Meteorol. Soc., 137, 829-844, 2011.

Nicholls, M. E. and Montgomery, M. T.: An examination of two pathways to tropical cyclogenesis occurring in idealized simulations with a cloud-resolving numerical model, Atmos. Chem. Phys., 13, 5999-6022, doi:10.5194/acp-13-5999-2013, 2013. 
Nolan, D. S. and Grasso, L. D.: Nonhydrostatic, three-dimensional perturbations to balanced, hurricane-like vortices. Part II: Symmetric response and nonlinear simulations, J. Atmos. Sci., 60, 2717-2745, 2003.

Nolan, D. S., Yumin, M., and Stern, D. P.: Tropical cyclone intensification from asymmetric convection: Energetics and efficiency, J. Atmos. Sci., 64, 3377-3405, 2007.

Nolan, D. S., Stern, D. P., and Zhang, J. A.: Evaluation of planetary boundary layer parameterizations in tropical cyclones by comparison of in situ observations and high-resolution simulations of Hurricane Isabel (2003). Part II: Inner-core boundary layer and eyewall structure, Mon. Weather Rev., 137, 3675-3698, 2009a.

Nolan, D. S., Zhang, J. A., and Stern, D. P.: Evaluation of planetary boundary layer parameterizations in tropical cyclones by comparison of in situ observations and high-resolution simulations of Hurricane Isabel (2003). Part I: Initialization, maximum winds, and the outer-core boundary layer, Mon. Weather Rev., 137, 3651-3674, 2009b.

Ooyama, K. V.: Numerical simulation of the life cycle of tropical cyclones, J. Atmos. Sci., 26, 3-40, 1969.

Ooyama, K. V.: Conceptual evolution of the theory and modeling of the tropical cyclone. Journal of the Meteorological Society of Japan, 60, 369-380, 1982.

Persing, J. and Montgomery, M. T.: Hurricane superintensity, J. Atmos. Sci., 60, 2349-2371, 2003.

Persing, J. and Montgomery, M. T.: Is environmental CAPE important in the determination of maximum possible hurricane intensity?, J. Atmos. Sci., 62, 542-550, 2005

Powell, M. D., Vickery, P. J., and Reinhold, R. A.: Reduced drag coefficient for high wind speeds in tropical cyclones, Nature, 422, 279-283, 2003.

Powell, M. D., Ulhorn, E. W., and Kepert, J. D.: Estimating maximum surface winds from hurricane reconnaissance measurements, Weather Forecast., 24, 868-883, 2009.

Raymond, D. J. and López Carrillo, C.: The vorticity budget of developing typhoon Nuri (2008), Atmos. Chem. Phys., 11, 147**163, doi:10.5194/acp-11-147-2011, 2011.

Reasor, P. D., Montgomery, M. T., and Bosart, L. F.: Mesoscale observations of the genesis of Hurricane Dolly (1996), J. Atmos. Sci., 62, 3151-3171, 2005.

Rotunno, R.: Study in tornado-like vortex dynamics, J. Atmos. Sci., 36, 140-155, 1979.

Rotunno, R. and Bryan, G. H.: Effects of parameterized diffusion on simulated hurricanes, J. Atmos. Sci., 69, 2284-2299, 2012.

Rotunno, R. and Emanuel, K. A.: An air-sea interaction theory for tropical cyclones. Parti II: Evolutionary study using a nonhydrostatic axisymmetric numerical model, J. Atmos. Sci., 44, 542$561,1987$.

Rozoff, C. M., Schubert, W. H., McNoldy, B., and Kossin, J. P.: Rapid filamentation zones in intense tropical cyclones, J. Atmos. Sci., 63, 325-340, 2006.

Sanger, N. T., Montgomery, M. T., Smith, R. K., and Bell, M. M.: An observational study of tropical cyclone spin-up in Supertyphoon Jangmi (2009) from 24-27 September, Mon. Weather Rev., in press, 2013.

Schecter, D. A.: Evaluation of a reduced model for investigating hurricane formation from turbulence, Q. J. R. Meteorol. Soc., 137, 155-178, 2011.
Schubert, W. H., Montgomery, M. T., Taft, R. K., Guinn, T. A., Fulton, S. R., Kossin, J. P., and Edwards, J. P.: Polygonal eyewalls, asymmetric eye contraction, and potential vorticity mixing in hurricanes, J. Atmos. Sci., 56, 1197-1223, 1999.

Shapiro, L. J. and Montgomery, M. T.: A three-dimensional balance theory for rapidly rotating vortices, J. Atmos. Sci., 50, 33223335, 1993.

Shapiro, L. J. and Willoughby, H. E.: The response of balanced hurricanes to local sources of heat and momentum, J. Atmos. Sci., 39, 378-394, 1982.

Shin, S. and Smith, R. K.: Tropical-cyclone intensification and predictability in a minimal three dimensional model, Q. J. R. Meteorol. Soc., 134, 1661-1671, 2008.

Sippel, J. A., Nielsen-Gammon, J. W., and Allen, S. E.: The multiple-vortex nature of tropical cyclogenesis, Mon. Weather Rev., 134, 1796-1814, 2006.

Smagorinsky, J.: General circulation experiments with the primitive equations. I: The basic experiment, Mon. Weather Rev., 91, 99164, 1963.

Smith II, G. B. and Montgomery, M. T.: Vortex axisymmetrization: Dependence on aximuthal wave-number or asymmetric radial structure changes, Q. J. R. Meteorol. Soc., 121, 1615-1650, 1995.

Smith, R. K. and Montgomery, M. T.: Hurricane boundary-layer theory, Q. J. R. Meteorol. Soc., 136, 1665-1670, 2010.

Smith, R. K. and Thomsen, G. L.: Dependence of tropical cyclone intensification on the boundary layer representation in a numerical model, Q. J. R. Meteorol. Soc., 136, 1671-1685, 2010.

Smith, R. K. and Vogl, S.: A simple model of the hurricane boundary layer revisted, Q. J. R. Meteorol. Soc., 134, 337-351, 2008.

Smith, R. K., Montgomery, M. T., and Nguyen, S. V.: Axisymmetric dynamics of tropical cyclone intensification in a threedimensional model, Q. J. R. Meteorol. Soc., 134, 337-351, 2008.

Smith, R. K., Montgomery, M. T., and Nguyen, S. V.: Tropical cyclone spin-up revisited, Q. J. R. Meteorol. Soc., 135, 1321-1335, 2009.

Smith, R. K., Montgomery, M. T., and Schmidt, C.: Dynamical constraints on the intensity and size of tropical cyclones, Q. J. R. Meteorol. Soc., 137, 1841-1855, 2011.

Smith, R. K., Montgomery, M. T., and Persing, J.: On steady-state tropical cyclones, Q. J. R. Meteorol. Soc., submitted, 2013a.

Smith, R. K., Montgomery, M. T., and Thomsen, G. L.: Sensitivity of tropical cyclone models to the surface drag coefficient in different boundary-layer schemes, Q. J. Roy. Meteorol. Soc., doi:10.1002/qj.2057, 2013 b.

Sprague, M., Julien, K., Knobloch, E., and Werne, J.: Numerical simulation of an asymptotically reduced system for rotationally constrained convection, J. Fluid Mechanics, 551, 141-174, 2006.

Sutyrin, G. G.: Azimuthal waves and symmetrization of an intense vortex, Sov. Phys. Dokl., 34, 104-106, 1990.

Thomsen, G. L., Smith, R. K., and Montgomery, M. T.: Tropicalcyclone flow asymmetries induced by a uniform flow revisited, Q. J. R. Meteorol. Soc., in review, 2013.

Vigh, J. L. and Schubert, W. H.: Rapid development of the tropical cyclone warm core, J. Atmos. Sci., 66, 3335-3350, 2009.

Vladimirov, V. A. and Tarasov, V. F.: Turbulence structure near the core of a circular vortex. Doklady Akademii Nauk SSSR, 245, 1325-1328, 1979. 
Wang, Y.: Vortex Rossby waves in a numerically simulated tropical cyclone. Part I: Overall structure, potential vorticity, and kinetic energy budgets, J. Atmos. Sci., 59, 1213-1238, 2002 a.

Wang, Y.: Vortex Rossby waves in a numerically simulated tropical cyclone. Part II: The role in tropical cyclone structure and intensity changes, J. Atmos. Sci., 59, 1239-1262, 2002b.

Willoughby, H. E.: Forced secondary circulations in hurricanes, J. Geophys. Res., 84, 3173-3183, 1979.

Willoughby, H. E.: Gradient balance in tropical cyclones, J. Atmos. Sci., 47, 265-274, 1990.

Willoughby, H. E., Clos, J., and Shoreibah, M.: Concentric eye walls, secondary wind maxima, and the evolution of the hurricane vortex, J. Atmos. Sci., 39, 395-411, 1982.

Wissmeier, U. and Smith, R. K.: Tropical cyclone convection: The effects of ambient vertical vorticity, Q. J. R. Meteor. Soc., 137, 845-857, 2011.

Wu, L. and Braun, S. A.: Effects of environmentally induced asymmetries on hurricane intensity: A numerical study, J. Atmos. Sci., 61, 3065-3081, 2004.
Yang, B., Wang, Y., and Wang, B.: The effects of internally generated asymmetries on tropical cyclone potential intensity, J. Atmos. Sci., 64, 1165-1188, 2007.

Zhang, D.-L., Liu, Y., and Yau, M. K.: A multiscale numerical study of Hurricane Andrew (1992). Part IV: Unbalanced flows, Mon. Weather Rev., 129, 92-107, 2001.

Zhang, J. A. and Drennan, W. A.: An observational study of vertical eddy diffusivity in the hurricane boundary layer, J. Atmos. Sci., 69, 3223-3236, 2012.

Zhang, J. A. and Montgomery, M. T.: Observational estimates of the horizontal eddy diffusivity and mixing length in the low-level region of intense hurricanes, J. Atmos. Sci., 69, 1306-1316, 2012.

Zhang, J. A., Drennan, W. M., Black, P. G., and French, J. R.: Turbulence structure of the hurricane boundary layer between the outer rainbands, J. Atmos. Sci., 66, 2455-2467, 2007.

Zhang, J. A., Rogers, R. F., Nolan, D. S., and Marks Jr., F. D.: On the characteristic height scales of the hurricane boundary layer, Mon. Weather Rev., 139, 2523-2535, 2011. 